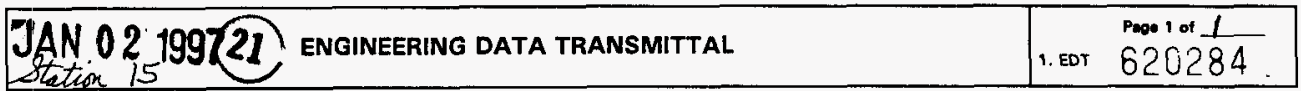

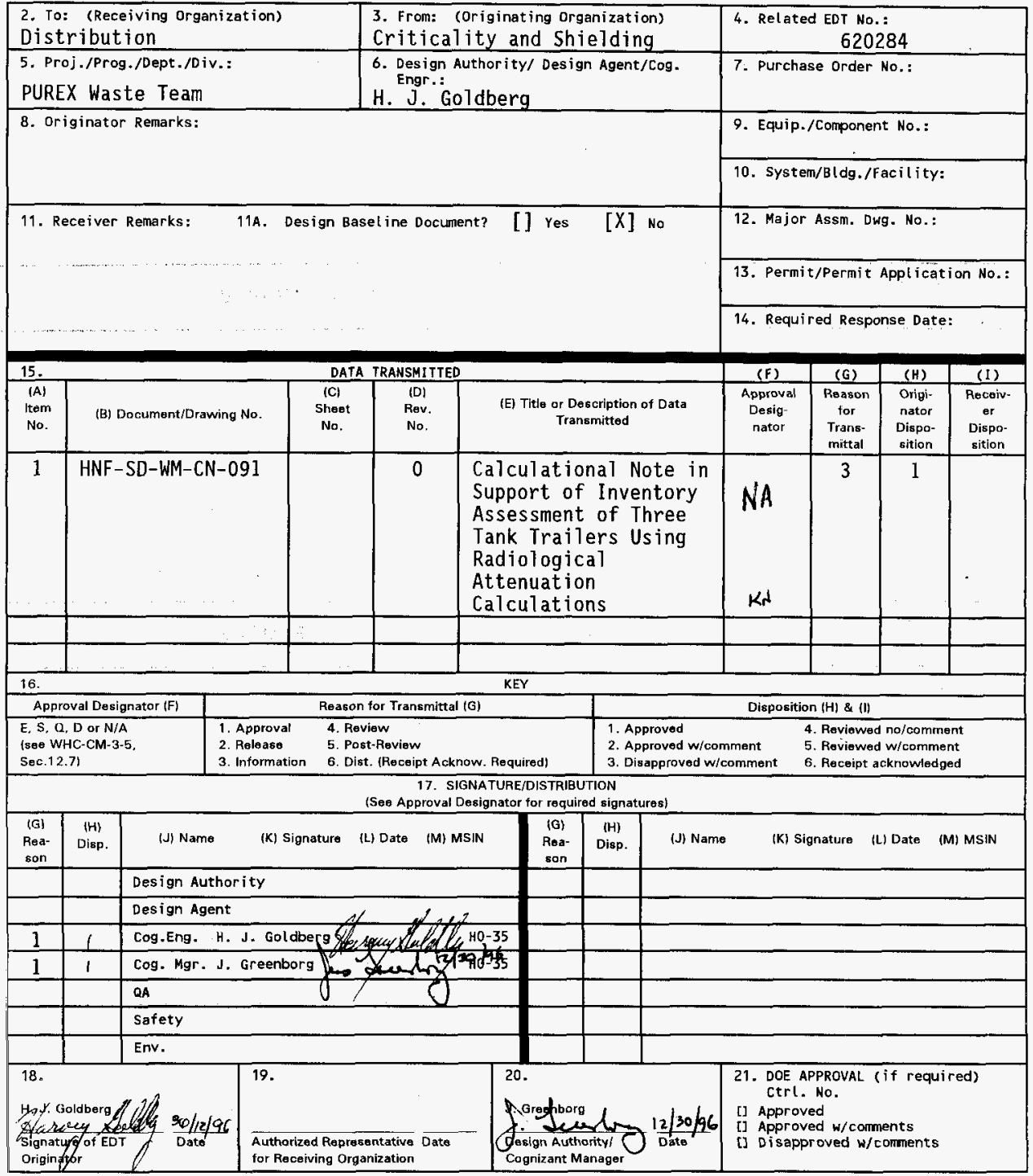

BD - 7400-172-2 (05/96) GEF097 


\section{Calculational Note in Support of Inventory Assessment of Three Tank Trailers Using Radiological Attenuation Calculations}

H. J. Goldberg

Fluor Daniel Northwest, Inc., Richland, WA 99352

U.S. Department of Energy Contract DE-AC06-96RL 13200

EDT/ECN: $\quad 620284$

Org Code: 403

B\&R Code: EW7002010
UC: 510

Charge Code: E51191

Total Pages: 6465

Key Words: Purex, UNH, Waste, Organic, Tanker

Abstract: An estimate of tanker inventory using radiological attenuation calculations.

TRADEMARK DISCLAIMER. Reference herein to any specific commercial product, process, or service by trade name, trademark, manufacturer, or otherwise, does not necessarily constitute or imply its endorsement, recommendation, or favoring by the United States Government or any agency thereof or its contractors or subcontractors.

Printed in the United States of America. To obtain copies of this document, contact: WHC/BCS Document Control Services, P.0. Box 1970, Mailstop H6-08, Richland WA 99352, Phone (509) 372-2420. Fax (509) 376-4989.
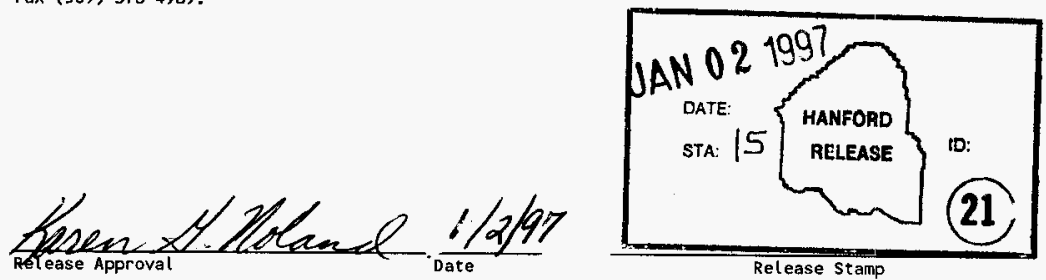

Approved for Public Release 
HNF-SD-WM-CN-091

Rev. 0

Page 1 of 64

\section{Calculational Note in support of \\ Inventory Assessment of Three Tank Trailers using Radiological Attenuation Calculations}

Harvey Goldberg

21 December 1996

\section{Purpose}

The PUREX Plant wishes to ascertain the degree of radiological contamination inside three tank trailers which are, for all extent and purposes, empty. Dose/Exposure rate measurements have been performed on the outside of the tanks which, when combined with historical records of the isotopic composition of the waste that had been shipped in the tanks, provided an estimate of the present degree of contamination.

\section{Methodology}

The computer code ISOSHLD (References 1, 2, and 3) was used to calculate the dose rate on the outside of the tanks. Bremsstrahlung from the B-particle emitting radionuclides was accounted for. The fluence-to-dose conversion factors used in the calculation were the deep dose equivalent conversion factors from Reference 4 . It was felt that these conversion factors corresponded best to the calibration of the Hanford hand-held survey instruments.

\section{Assumptions}

It was assumed that each tank could be modeled as a long, thin cylinder with cylindrical shields between it and the dose points. Dose points were chosen in the middie and the end of the side of the tank to correspond to the radiological survey provided by the customer. The first shield which is the source was modeled somewhat arbitrarily as aluminum.

The density used was the reported density of the original solutions although by this time evaporation most probably has resulted in a much denser solution. This is a conservative assumption with respect to the various fission products and uranium actually in the source. The second shield was chosen to be iron which is again a conservative assumption since stainless steel is slightly denser. 
HNF-SD-WM-CN-091

Rev. 0

Page 2 of 64

\section{Input Data}

There are three tanker trailers being investigated;

- H0-64-4278, formerly used for organic waste. This trailer is $1036 \mathrm{~cm}$ $(\sim 34 \mathrm{ft})$ Tong and has an outer diameter of $12.70 \mathrm{~cm}(\sim 5 \mathrm{ft})$. It is constructed out of $\frac{1}{2}$ in 316 stainless steel. The tanker has a capacity of approximately $18,9001(5,000 \mathrm{gal})$ although it is estimated that there is only 3.8 to $7.61(1-2$ gal $)$ of waste in the tanker.

- H0-64-5920, an older tanker used for Uranyl Nitrate Hexahydrate (UNH). This trailer is $701 \mathrm{~cm}(\sim 23 \mathrm{ft})$ long and has an outer diameter of $145 \mathrm{~cm}$ ( $57 \mathrm{in})$. It is constructed out of $\frac{1}{4}$ in $304 \mathrm{~L}$ stainless steel. The tanker has a capacity of approximately $11,4001(3,000 \mathrm{gal})$ although it is estimated that it is empty at the moment. However there may be a solid heel on the bottom.

- H0-64-5473, a newer tanker used for UNH. This trailer is $751 \mathrm{~cm}$ ( $24 \mathrm{ft} 7.5 \mathrm{in})$ long and has an outer diameter of $145 \mathrm{~cm}(\sim 57 \mathrm{in})$. It is constructed out of $\frac{1}{4}$ in $304 \mathrm{~L}$ stainless steel. The tanker has a capacity of approximately $11,4001(3,000 \mathrm{gal})$ although it is estimated that there is only 7.6 to $11.41(2-3 \mathrm{gal})$ of waste in the tanker.

Surveys have been performed. The contact exposure rates measured were 170 $\mathrm{mR} / \mathrm{hr}$ at the "belly" of the organic tank. It was assumed that this measurement was performed in the center of the tanker. In addition a measurement at the front yielded $3 \mathrm{mR} / \mathrm{hr}$ while one at the back yielded 4 $\mathrm{mR} / \mathrm{hr}$. These were also in contact with the surface of the tank. Exposure rates along the surface of both UNH tanks were below $0.5 \mathrm{mR} / \mathrm{hr}$. For the purpose of this analysis, the conservative assumption was made that the exposure rates were at this limit.

An isotopic inventory of the solutions shipped in the tankers was used as the assumed isotopic inventory of the remaining contamination. In the case of the organic waste, two analyses were available. The worst case inventory was chosen. These inventories are presented below in Table 1. 
HNF-SD-WM-CN-091

Rev. 0

Page 3 of 64

\begin{tabular}{|c|c|c|}
\hline \multicolumn{3}{|c|}{ 1able 1. isoloptc inventory $(201 / 1)$} \\
\hline Isotope & $\begin{array}{c}\text { Organic Waste } \\
\rho=0.8122 \mathrm{~g} / \mathrm{cm}^{3}\end{array}$ & $\rho=1.665 \mathrm{~g} / \mathrm{cm}^{3}$ \\
\hline${ }^{60} \mathrm{Co}$ & $1.45 e-01$ & \\
\hline${ }^{95} \mathrm{Nb}$ & $1.84 \mathrm{e}-02$ & $4.67 e-01$ \\
\hline${ }^{95} \mathrm{Zr}$ & $2.08 \mathrm{e}-02$ & $7.18 \mathrm{e}-02$ \\
\hline${ }^{103} \mathrm{Ru}$ & $1.46 \mathrm{e}-02$ & $<3.96 \mathrm{e}-02$ \\
\hline${ }^{106} \mathrm{Ru}$ & $6.82 \mathrm{e}+00$ & $<5.33 \mathrm{e}-01$ \\
\hline${ }^{106} \mathrm{Rh}$ & $6.82 \mathrm{e}+00$ & $<5.33 \mathrm{e}-01$ \\
\hline${ }^{125} \mathrm{Sb}$ & $1.20 e-01$ & \\
\hline${ }^{134} \mathrm{Cs}$ & $1.93 \mathrm{e}-02$ & $4.27 e-02$ \\
\hline${ }^{137} \mathrm{Cs}$ & $8.45 e-01$ & $3.60 \mathrm{e}-02$ \\
\hline${ }^{137 m} \mathrm{Ba}$ & $7.99 \mathrm{e}-01$ & $3.41 \mathrm{e}-02$ \\
\hline${ }^{144} \mathrm{Ce}$ & $7.12 \mathrm{e}-01$ & $<5.81 \mathrm{e}-01$ \\
\hline${ }^{144} \mathrm{Pr}$ & $7.12 \mathrm{e}-01$ & $<5.81 \mathrm{e}-01$ \\
\hline${ }^{155} \mathrm{Eu}$ & $8.44 \mathrm{e}-02$ & \\
\hline${ }^{234} \mathrm{U}$ & $1.72 \mathrm{e}-09$ & $2.69 e+03$ \\
\hline${ }^{235} \mathrm{U}$ & $4.97 e-08$ & $9.80 \mathrm{e}-02$ \\
\hline${ }^{236} \mathrm{U}$ & $1.39 e-08$ & $2.17 \mathrm{e}-02$ \\
\hline${ }^{238} \mathrm{U}$ & $8.31 \mathrm{e}-06$ & $1.59 \mathrm{e}+00$ \\
\hline${ }^{241} \mathrm{Am}$ & $1.50 \mathrm{e}-01$ & \\
\hline
\end{tabular}

Whenever the concentration is reported as less than an amount, the amount was used as a worst case. The Uranium numbers were calculated from the gram amounts as illustrated in Table 2. The UNH waste had $4.7905 \mathrm{~g}_{\mathrm{v}} / 1$ while the Organic waste had $2.500 \times 10^{-6} \mathrm{~g}_{\mathrm{u}} / 1$. 
HNF-SD-WM-CN-091

Rev. 0

Page 4 of 64

\begin{tabular}{||c|c|c|c|c|c||}
\hline Isotope & $\begin{array}{c}\mathrm{S} \mathrm{A} * \\
(\mu \mathrm{C} / \mathrm{g})\end{array}$ & $\begin{array}{c}\text { Waste } \\
\text { Type }\end{array}$ & $\begin{array}{c}\text { weight } \\
\text { fraction }\end{array}$ & $\begin{array}{c}\text { Weight } \\
\text { Concentration } \\
(\mathrm{g} / 1)\end{array}$ & $\begin{array}{c}\text { Activity } \\
\text { Concentration } \\
(\mu \mathrm{C} \mathrm{j} / \mathrm{1})\end{array}$ \\
\hline \hline${ }^{234} \mathrm{U}$ & $6.25 \mathrm{e}+03$ & Organic & 0.00011 & $2.75 \mathrm{e}-10$ & $1.72 \mathrm{e}-06$ \\
\cline { 3 - 7 } & UNH & 0.00009 & $4.31 \mathrm{e}-04$ & $2.69 \mathrm{e}+00$ \\
\hline${ }^{235} \mathrm{U}$ & \multirow{2}{*}{$2.16 \mathrm{e}+00$} & Organic & 0.00921 & $2.30 \mathrm{e}-08$ & $4.97 \mathrm{e}-08$ \\
\cline { 3 - 7 } & & UNH & 0.00947 & $4.54 \mathrm{e}-02$ & $9.80 \mathrm{e}-02$ \\
\hline${ }^{236} \mathrm{U}$ & \multirow{2}{*}{$6.47 \mathrm{e}+01$} & Organic & 0.00086 & $2.15 \mathrm{e}-09$ & $1.39 \mathrm{e}-07$ \\
\cline { 3 - 7 } & & UNH & 0.0007 & $3.35 \mathrm{e}-03$ & $2.17 \mathrm{e}-01$ \\
\hline${ }^{238} \mathrm{U}$ & \multirow{2}{*}{$3.36 \mathrm{e}-01$} & Organic & 0.98982 & $2.47 \mathrm{e}-06$ & $8.31 \mathrm{e}-07$ \\
\cline { 3 - 7 } & & UNH & 0.98974 & $4.74 \mathrm{e}+00$ & $1.59 \mathrm{e}+00$ \\
\hline
\end{tabular}

* Reference 5

\section{Calculations}

The computer code ISOSHLD (References 1, 2, and 3) was used to calculate the dose rate on the outside of the tanks. Bremsstrahlung from the B-particle emitting radionuclides was accounted for. The fluence-to-dose conversion factors used in the calculation were the deep dose equivalent conversion factors from Reference 4 . It was felt that these conversion factors corresponded best to the calibration of the Hanford hand-held survey instruments.

The source was modeled as a long thin cylinder, corresponding to a solid heel or a few gallons of waste on the lowest portion of the tanker. An annular source was experimented with also which would correspond to a thin layer of contamination covering all of the inside surface of the tanker. However, it was felt that the results of this calculation were unbelievable.

The dose points were in contact with the outside surface of the tanker in the middle of its length and at the end of the side wall. More credence was placed in the midpoint readings than in the end readings since the effect of the end wall and of any additional hardware on the end of the tanker could not be taken into account.

The activity of one liter of original solution was used as a source. This source was multiplied by 1,000 in order to have the results in $\mathrm{mR} / \mathrm{hr}$ rather than $\mathrm{R} / \mathrm{hr}$. These results are tabulated in Table 3. 
HNF-SD-WM-CN-091

Rev. 0

Page 5 of 64

\begin{tabular}{|c|c|c|c|c|}
\hline Source & Position & $\begin{array}{l}\text { ISOSHLD } \\
(\mathrm{mR} / \mathrm{hr})\end{array}$ & $\begin{array}{c}\text { Measurement } \\
(\mathrm{mR} / \mathrm{hr})\end{array}$ & $\begin{array}{c}\text { Estimated } \\
\text { Volume } \\
\text { (1) }\end{array}$ \\
\hline \multirow{2}{*}{$\begin{array}{c}\text { Organic } \\
\text { Tanker }\end{array}$} & Middle & $1.03 \times 10^{-2}$ & 17 & 1600 \\
\hline & End & $7.20 \times 10^{-3}$ & $\begin{array}{c}3 \text { (back) } \\
4 \text { (Front) }\end{array}$ & $\begin{array}{c}420 \\
560\end{array}$ \\
\hline \multirow{2}{*}{$\begin{array}{l}\text { 01d UNH } \\
\text { Tanker }\end{array}$} & Middle & $6.65 \times 10^{-3}$ & $<0.5$ & $<75$ \\
\hline & End & $4.79 \times 10^{-3}$ & $<0.5$ & $<100$ \\
\hline \multirow{2}{*}{$\begin{array}{l}\text { New UNH } \\
\text { Tanker }\end{array}$} & Middle & $6.21 \times 10^{-3}$ & $<0.5$ & $<81$ \\
\hline & End & $4.48 \times 10^{-3}$ & $<0.5$ & $<110$ \\
\hline
\end{tabular}

As I stated above, the middle reading is more believable than the end reading, and thus in my opinion, the best estimate of the amount of radionuclides in the tankers is 16001 in the organic tanker, 751 in the old UNH tanker, and 811 in the new UNH tanker. It must be understood that this is the activity that was in those volumes of the original solution, not the actual volume of residue left in the tanks. This activity is definitely more concentrated due to evaporation of the original waste solutions and may be in the form of a solid residue.

\section{Conclusions}

Table 4 is a listing of the estimated activities of the various isotopes bel ieved to be in each of the three tankers. In addition, the uranium estimates are given in terms of estimated mass as well as estimated activity. 
HNF-SD-WM-CN-091

Rev. 0

Page 6 of 64

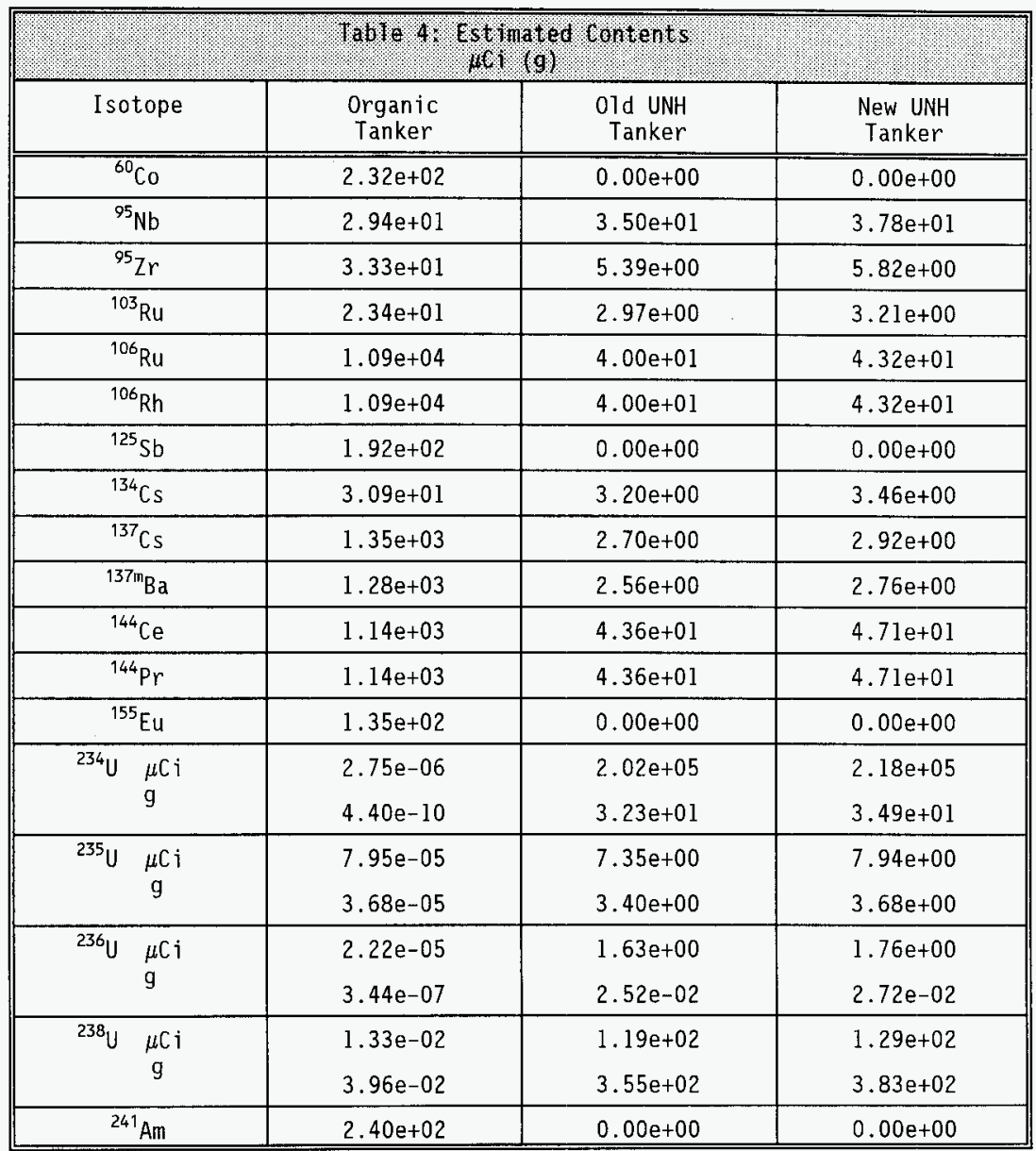




$$
\begin{array}{r}
\text { HNF-SD-WM-CN-091 } \\
\text { Rev. } 0 \\
\text { Page } 7 \text { of } 64
\end{array}
$$

The total uranium content of the three tankers is $4.0 \times 10^{-2} \mathrm{~g}$ for the organic tanker, $3.9 \times 10^{2} \mathrm{~g}$ for the old UNH tanker, and $4.2 \times 10^{2} \mathrm{~g}$. Note that these are worst case estimates. This is especially true for the UNH tanks. The survey sheets indicate that no readings were obtained and the readings are recorded as less than the lower limit of detection.

\section{REFERENCES}

1) ISOSHLD- A Computer Code for General Purpose Isotope Shielding Analysis, BNWL-236, R. L. Enge1, J. Greenborg, M. M. Hendrickson, dated June 1966.

2) ISOSHLD-II: Code Revision to Include Calculation of Dose Rate from Shielded Bremsstrahlung Sources, G. L. Simmons, J. J. Regimbal, J. Greenborg, E. L. Kelly, Jr., H. H. van Tuyl, dated March 1967.

3) CCC-636, ISO-PC Version 2.1, WHC-SD-WM-UM-030, P. D. Rittmann, Radiation Safety Information Computational Center, 0ak Ridge, Tennessee, 1996.

4) Neutron and Gamma-ray Fluence-to-dose Factors, ANSI/ANS-6.1.1-1977.

5) Basis and Values for Specific Activity and Decay Heat Generation Rates for Selected Radionuclides, SD-RE-TI-131, T. D. Kirkpatric and R. C. Brown, Rockwe 11 Hanford Operations, Richland, Washington, 1984 


\section{HNF-SD-WM-CN-091 \\ Rev. 0 \\ Page 8 of 64}

Run started at $11: 54: 57 \quad 12 / 20 / 96$

\section{Computer output}

ISO-PC Version 2.1 February 1996

originally ISOSHLD-II; RIBD was removed

Please send questions or comments to:

Pau1 D. Rittmann, PhD CHP 509-376-8715

Westinghouse Hanford Company H6-06

P0 Box $1970 \quad$ Richland, WA 99352

eMail: Paul_D_Rittmannorl.gov

Title Line from Library File (ISO-PC.LIB):

Attenuation \& Buildup for 30 Groups; Photon \& Beta Production 2/14/96 PDR

Run Title: PUREX Tank Trailers

Organic Tank (HO-64-4278) Line Source - Middle

Table of Source Activity:

Scale Factor $=1.000 E+03$

\begin{tabular}{|c|c|c|c|}
\hline \multicolumn{2}{|c|}{ I sotope } & Initial & Final \\
\hline 472 & $\mathrm{CO}-60$ & $1.450 \mathrm{E}-01$ & $1.450 E+02$ \\
\hline 117 & ZR- 95 & $2.080 \mathrm{E}-02$ & $2.080 E+01$ \\
\hline 119 & NB- 95 & $1.840 \mathrm{E}-02$ & $1.840 E+01$ \\
\hline 155 & RU-103 & $1.460 \mathrm{E}-02$ & $1.460 \mathrm{E}+01$ \\
\hline 170 & RU-106 & $6.820 E+00$ & $6.820 \mathrm{E}+03$ \\
\hline 172 & RH-106 & $6.820 E+00$ & $6.820 E+03$ \\
\hline 269 & $S B-125$ & $1.200 \mathrm{E}-01$ & $1.200 \mathrm{E}+02$ \\
\hline 319 & CS-134 & $1.930 \mathrm{E}-02$ & $1.930 \mathrm{E}+01$ \\
\hline 335 & CS-137 & $8.450 \mathrm{E}-01$ & $8.450 \mathrm{E}+02$ \\
\hline 336 & $B A-137 M$ & $7.990 \mathrm{E}-01$ & $7.990 \mathrm{E}+02$ \\
\hline 376 & CE-144 & $7.120 \mathrm{E}-01$ & $7.120 \mathrm{E}+02$ \\
\hline 377 & PR-144 & $7.120 \mathrm{E}-01$ & $7.120 \mathrm{E}+02$ \\
\hline 418 & EU-155 & $8.440 \mathrm{E}-02$ & $8.440 \mathrm{E}+01$ \\
\hline 520 & $U-234$ & $1.720 \mathrm{E}-09$ & $1.720 \mathrm{E}-06$ \\
\hline 476 & $U-235$ & $1.390 \mathrm{E}-08$ & $1.390 \mathrm{E}-05$ \\
\hline 398 & $U-236$ & $4.970 \mathrm{E}-08$ & $4.970 \mathrm{E}-05$ \\
\hline 526 & $U-238$ & $8.310 E-06$ & $8.310 \mathrm{E}-03$ \\
\hline 496 & $A M-241$ & $2.150 \mathrm{E}-02$ & $2.150 \mathrm{E}+01$ \\
\hline
\end{tabular}


HNF-SD-WM-CN-091

Rev. 0

Page 9 of 64

Organic Tank (H0-64-4278) Line Source - Middle

Photon Production Rate for Each Radionuclide:

\begin{tabular}{|c|c|c|c|c|c|c|}
\hline $\begin{array}{l}\gg>C O \\
\text { Group } \\
\text { No. }\end{array}$ & $\begin{array}{l}60 \\
\text { Photon } \\
\text { Mean }\end{array}$ & $\begin{array}{c}Z=27) \\
\text { Energy } \\
\text { Low }\end{array}$ & $\begin{array}{l}\text {, MeV } \\
\mathrm{High}\end{array}$ & $\begin{array}{l}\text { eight ( } 472) \\
\text { Photon Pro } \\
\text { Gamma\&Xray }\end{array}$ & $\begin{array}{c}1.450 E+02 \\
\text { uction Rate } \\
\text { Bremss. }\end{array}$ & $\begin{array}{l}\text { Total } \\
\text { photon/s }\end{array}$ \\
\hline 1 & 0.0144 & 0.01 & 0.02 & $.000 \mathrm{E}+00$ & $1.701 E+04$ & $1.701 E+04$ \\
\hline 2 & 0.0241 & 0.02 & 0.03 & $.000 E+00$ & $5.881 E+03$ & $5.881 E+03$ \\
\hline 3 & 0.0345 & 0.03 & 0.04 & $0.000 E+00$ & $3.187 E+03$ & $3.187 \bar{E}+03$ \\
\hline 4 & 0.0447 & 0.04 & 0.05 & $0.000 E+00$ & $1.877 \mathrm{E}+03$ & $1.877 \mathrm{E}+03$ \\
\hline 5 & 0.0546 & 0.05 & 0.06 & $0.000 E+00$ & $1.211 E+03$ & $1.211 E+03$ \\
\hline 6 & 0.0647 & 0.06 & 0.07 & $0.000 \mathrm{E}+00$ & $8.433 E+02$ & $8.433 \mathrm{E}+02$ \\
\hline 7 & 0.0747 & 0.07 & 0.08 & $0.000 \mathrm{E}+00$ & $5.828 E+02$ & $5.828 \mathrm{E}+02$ \\
\hline 8 & 0.0847 & 0.08 & 0.09 & $0.000 E+00$ & $4.164 E+02$ & $4.164 E+02$ \\
\hline 9 & 0.0947 & 0.09 & 0.10 & $0.000 \mathrm{E}+00$ & $3.022 E+02$ & $3.022 E+02$ \\
\hline 10 & 0.1239 & 0.10 & 0.20 & $0.000 \mathrm{E}+00$ & $7.872 \mathrm{E}+02$ & $7.872 E+02$ \\
\hline 11 & 0.2141 & 0.20 & 0.30 & $0.000 \mathrm{E}+00$ & $2.098 E+01$ & 2.09 \\
\hline 12 & 0.3000 & 0.30 & 0.40 & $0.000 \mathrm{E}+00$ & $8.216 E-03$ & $8.216 \mathrm{E}-03$ \\
\hline 14 & 0.6938 & 0.55 & 0.75 & $8.584 E+02$ & $0.000 E+00$ & $8.584 E+02$ \\
\hline 17 & 1.2530 & 1.10 & 1.35 & $1.073 E+07$ & $0.000 E+00$ & $1.073 \mathrm{E}+07$ \\
\hline
\end{tabular}

$\gg$ PR- $95 \quad(Z=40) \quad$ Weight $(117)=2.080 \mathrm{E}+01 \mu \mathrm{Ci}$

Group Photon Energy, MeV Photon Production Rate Total

No. Mean Low High Gamma\&Xray Bremss. photon/s

\begin{tabular}{rllllll}
\hline 1 & 0.0145 & 0.01 & 0.02 & $0.000 \mathrm{E}+00$ & $2.867 \mathrm{E}+03$ & $2.867 \mathrm{E}+03$ \\
2 & 0.0242 & 0.02 & 0.03 & $0.000 \mathrm{E}+00$ & $1.338 \mathrm{E}+03$ & $1.338 \mathrm{E}+03$ \\
3 & 0.0347 & 0.03 & 0.04 & $0.000 \mathrm{E}+00$ & $6.166 \mathrm{E}+02$ & $6.166 \mathrm{E}+02$ \\
4 & 0.0446 & 0.04 & 0.05 & $0.000 \mathrm{E}+00$ & $3.882 \mathrm{E}+02$ & $3.882 \mathrm{E}+02$ \\
5 & 0.0547 & 0.05 & 0.06 & $0.000 \mathrm{E}+00$ & $2.596 \mathrm{E}+02$ & $2.596 \mathrm{E}+02$ \\
6 & 0.0647 & 0.06 & 0.07 & $0.000 \mathrm{E}+00$ & $1.787 \mathrm{E}+02$ & $1.787 \mathrm{E}+02$ \\
7 & 0.0748 & 0.07 & 0.08 & $0.000 \mathrm{E}+00$ & $1.298 \mathrm{E}+02$ & $1.298 \mathrm{E}+02$ \\
8 & 0.0847 & 0.08 & 0.09 & $0.000 \mathrm{E}+00$ & $9.554 \mathrm{E}+01$ & $9.554 \mathrm{E}+01$ \\
9 & 0.0948 & 0.09 & 0.10 & $0.000 \mathrm{E}+00$ & $7.200 \mathrm{E}+01$ & $7.200 \mathrm{E}+01$ \\
10 & 0.1288 & 0.10 & 0.20 & $0.000 \mathrm{E}+00$ & $2.248 \mathrm{E}+02$ & $2.248 \mathrm{E}+02$ \\
11 & 0.2290 & 0.20 & 0.30 & $0.000 \mathrm{E}+00$ & $1.791 \mathrm{E}+01$ & $1.791 \mathrm{E}+01$ \\
12 & 0.3347 & 0.30 & 0.40 & $0.000 \mathrm{E}+00$ & $2.054 \mathrm{E}+00$ & $2.054 \mathrm{E}+00$ \\
13 & 0.4551 & 0.40 & 0.55 & $0.000 \mathrm{E}+00$ & $7.270 \mathrm{E}-01$ & $7.270 \mathrm{E}-01$ \\
14 & 0.7242 & 0.55 & 0.75 & $3.363 \mathrm{E}+05$ & $1.455 \mathrm{E}-01$ & $3.363 \mathrm{E}+05$ \\
115 & 0.7567 & 0.75 & 0.90 & $4.256 \mathrm{E}+05$ & $8.727 \mathrm{E}-03$ & $4.256 \mathrm{E}+05$ \\
16 & 0.9332 & 0.90 & 1.10 & $0.000 \mathrm{E}+00$ & $7.211 \mathrm{E}-04$ & $7.211 \mathrm{E}-04$ \\
17 & 1.1000 & 1.10 & 1.35 & $0.000 \mathrm{E}+00$ & $7.298 \mathrm{E}-08$ & $7.298 \mathrm{E}-08$ \\
\hline
\end{tabular}

Total Photons/sec: $7.619 \mathrm{E}+05 \quad 6.190 \mathrm{E}+03 \quad 7.681 \mathrm{E}+05$ 
HNF-SD-WM-CN-091

Rev. 0

Page 10 of 64

\begin{tabular}{|c|c|c|c|c|c|c|}
\hline $\begin{array}{l}\gg \text { NB- } \\
\text { Group } \\
\text { No. }\end{array}$ & $\begin{array}{l}95 \\
\text { Photon } \\
\text { Mean }\end{array}$ & $\begin{array}{c}Z=41 \text { ) } \\
\text { Energy, } \\
\text { Low }\end{array}$ & $\begin{array}{l}\text { MeV } \\
\text { High }\end{array}$ & $\begin{array}{l}\text { eight (119) } \\
\text { Photon Proc } \\
\text { Gamma\&Xray }\end{array}$ & $\begin{array}{l}1.840 E+0 \\
\text { tion Ra } \\
\text { Bremss. }\end{array}$ & $\begin{array}{c}\text { Total } \\
\text { photon/s }\end{array}$ \\
\hline $\begin{array}{r}1 \\
2 \\
3 \\
4 \\
5 \\
6 \\
7 \\
8 \\
9 \\
10 \\
11 \\
12 \\
15\end{array}$ & $\begin{array}{l}0.0136 \\
0.0244 \\
0.0344 \\
0.0444 \\
0.0545 \\
0.0645 \\
0.0745 \\
0.0844 \\
0.0944 \\
0.1003 \\
0.2144 \\
0.3000 \\
0.7658\end{array}$ & $\begin{array}{l}0.01 \\
0.02 \\
0.03 \\
0.04 \\
0.05 \\
0.06 \\
0.07 \\
0.08 \\
0.09 \\
0.10 \\
0.20 \\
0.30 \\
0.75\end{array}$ & $\begin{array}{l}0.02 \\
0.03 \\
0.04 \\
0.05 \\
0.06 \\
0.07 \\
0.08 \\
0.09 \\
0.10 \\
0.20 \\
0.30 \\
0.40 \\
0.90\end{array}$ & $\begin{array}{l}0.000 E+00 \\
0.000 E+00 \\
0.000 E+00 \\
0.000 E+00 \\
0.000 E+00 \\
0.000 E+00 \\
0.000 E+00 \\
0.000 E+00 \\
0.000 E+00 \\
0.000 E+00 \\
0.000 E+00 \\
0.000 E+00 \\
6.795 E+05\end{array}$ & $\begin{array}{l}6.098 \mathrm{E}+02 \\
1.963 \mathrm{E}+02 \\
8.983 \mathrm{E}+01 \\
4.550 \mathrm{E}+01 \\
2.414 \mathrm{E}+01 \\
1.285 \mathrm{E}+01 \\
6.746 \mathrm{E}+00 \\
3.465 \mathrm{E}+00 \\
1.707 \mathrm{E}+00 \\
2.772 \mathrm{E}+00 \\
8.258 \mathrm{E}-04 \\
4.805 \mathrm{E}-07 \\
0.000 \mathrm{E}+00\end{array}$ & $\begin{array}{l}6.098 \mathrm{E}+02 \\
1.963 \mathrm{E}+02 \\
8.983 \mathrm{E}+01 \\
4.550 \mathrm{E}+01 \\
2.414 \mathrm{E}+01 \\
1.285 \mathrm{E}+01 \\
6.746 \mathrm{E}+00 \\
3.465 \mathrm{E}+00 \\
1.707 \mathrm{E}+00 \\
2.772 \mathrm{E}+00 \\
8.258 \mathrm{E}-04 \\
4.805 \mathrm{E}-07 \\
6.795 \mathrm{E}+05\end{array}$ \\
\hline
\end{tabular}

Total Photons/sec: $\quad 6.795 E+05 \quad 9.931 E+02 \quad 6.805 E+05$

\begin{tabular}{|c|c|c|c|c|c|c|}
\hline $\begin{array}{c}\gg \mathrm{RU} \\
\text { Group } \\
\text { No. }\end{array}$ & $\begin{array}{l}103 \\
\text { Photon } \\
\text { Mean }\end{array}$ & $\begin{array}{l}=44 \text { ) } \\
\text { inergy } \\
\text { Low }\end{array}$ & $\begin{array}{c}\text { MeV } \\
\text { High }\end{array}$ & $\begin{array}{l}\text { Photon Proo } \\
\text { Gamma\&Xray }\end{array}$ & $\begin{array}{l}\text { ction Rat } \\
\text { Bremss. }\end{array}$ & $\begin{array}{c}\text { Total } \\
\text { photon/s }\end{array}$ \\
\hline $\begin{array}{r}1 \\
2 \\
3 \\
4 \\
5 \\
6 \\
7 \\
8 \\
9 \\
10 \\
11 \\
12 \\
13 \\
14\end{array}$ & $\begin{array}{l}0 . \\
0 . \\
0 . \\
0 . \\
0 . \\
0 . \\
0 . \\
0.2 \\
0 .\end{array}$ & $\begin{array}{l}0.01 \\
0.02 \\
0.03 \\
0.04 \\
0.05 \\
0.06 \\
0.07 \\
0.08 \\
0.09 \\
0.10 \\
0.20 \\
0.30 \\
0.40 \\
0.55\end{array}$ & $\begin{array}{l}0.04 \\
0.05 \\
0.06 \\
0.07 \\
0.08 \\
0.09 \\
0.10 \\
0.20 \\
0.30 \\
0.40 \\
0.55 \\
0.75\end{array}$ & $\begin{array}{l}0.000 E+00 \\
2.539 E+03 \\
0.000 E+00 \\
0.000 E+00 \\
1.945 E+03 \\
0.000 E+00 \\
0.000 E+00 \\
0.000 E+00 \\
0.000 E+00 \\
0.000 E+00 \\
1.459 E+03 \\
0.000 E+00 \\
4.773 E+05 \\
3.911 E+04\end{array}$ & $\begin{array}{l}1.551 \mathrm{E}+02 \\
8.891 \mathrm{E}+01 \\
5.052 \mathrm{E}+01 \\
3.336 \mathrm{E}+01 \\
2.256 \mathrm{E}+01 \\
1.501 \mathrm{E}+01 \\
1.055 \mathrm{E}+01 \\
3.251 \mathrm{E}+01 \\
5.263 \mathrm{E}+00 \\
1.251 \mathrm{E}+00 \\
2.963 \mathrm{E}-01 \\
1.611 \mathrm{E}-02\end{array}$ & $\begin{array}{l}7.374 \mathrm{E}+02 \\
2.825 \mathrm{E}+03 \\
1.551 \mathrm{E}+02 \\
8.891 \mathrm{E}+01 \\
1.995 \mathrm{E}+03 \\
3.336 \mathrm{E}+01 \\
2.256 \mathrm{E}+01 \\
1.501 \mathrm{E}+01 \\
1.055 \mathrm{E}+01 \\
3.251 \mathrm{E}+01 \\
1.464 \mathrm{E}+03 \\
1.251 \mathrm{E}+00 \\
4.773 \mathrm{E}+05 \\
3.911 \mathrm{E}+04\end{array}$ \\
\hline
\end{tabular}

Total Photons/sec: $5.223 \mathrm{E}+05 \quad 1.439 \mathrm{E}+03 \quad 5.238 \mathrm{E}+05$

$\gg$ RU-106 (Z $=44) \quad$ Weight $(170)=6.820 \mathrm{E}+03 \mu \mathrm{Ci}$

Group Photon Energy, MeV Photon Production Rate Total

No. Mean Low High Gamma\&Xray Bremss. photon/s

\begin{tabular}{rllllll}
1 & 0.0129 & 0.01 & 0.02 & $0.000 \mathrm{E}+00$ & $8.662 \mathrm{E}+03$ & $8.662 \mathrm{E}+03$ \\
2 & 0.0225 & 0.02 & 0.03 & $0.000 \mathrm{E}+00$ & $5.734 \mathrm{E}+02$ & $5.734 \mathrm{E}+02$ \\
3 & 0.0304 & 0.03 & 0.04 & $0.000 \mathrm{E}+00$ & $1.579 \mathrm{E}+01$ & $1.579 \mathrm{E}+01$ \\
\hline
\end{tabular}

Total Photons/sec: $\quad 0.000 \mathrm{E}+00 \quad 9.252 \mathrm{E}+03 \quad 9.252 \mathrm{E}+03$ 
HNF-SD-WM-CN-091

Rev. 0

Page 11 of 64

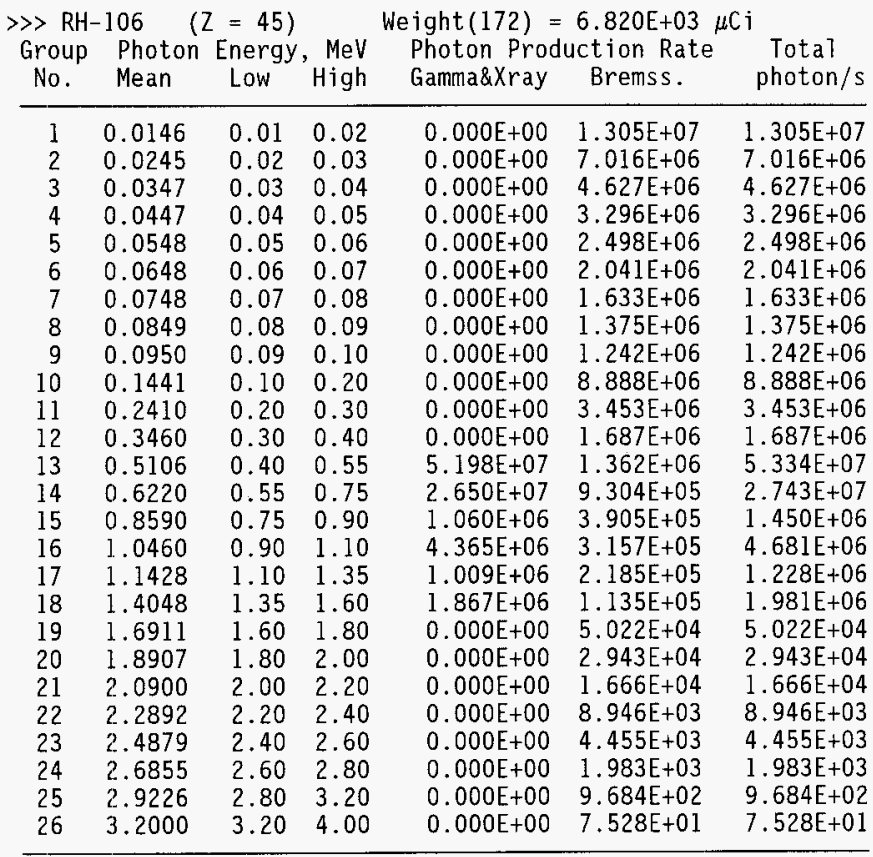

Total Photons/sec: $\quad 8.678 \mathrm{E}+07 \quad 5.425 \mathrm{E}+07 \quad 1.410 \mathrm{E}+08$ 
HNF-SD-WM-CN-091

Rev. 0

Page 12 of 64

\begin{tabular}{|c|c|c|c|c|c|c|}
\hline $\begin{array}{l}>\text { SB-1 } \\
\text { Group } \\
\text { No. }\end{array}$ & $\begin{array}{l}125 \quad(Z \\
\text { Photon } \\
\text { Mean }\end{array}$ & $\begin{array}{l}=51) \\
\text { Energy, } \\
\text { Low }\end{array}$ & $\begin{array}{r}\text { MeV } \\
\text { High }\end{array}$ & $\begin{array}{l}\text { ight }(269)= \\
\text { Photon Pro } \\
\text { Gamma\&Xray }\end{array}$ & $\begin{array}{l}\text { tion Rate } \\
\text { Bremss. }\end{array}$ & $\begin{array}{l}\text { Total } \\
\text { photon/s }\end{array}$ \\
\hline $\begin{array}{r}1 \\
2 \\
3 \\
4 \\
5 \\
6 \\
7 \\
8 \\
9 \\
10 \\
11 \\
12 \\
13 \\
14\end{array}$ & $\begin{array}{l}0.0287 \\
0.0345 \\
0.0445 \\
0.0547 \\
0.0647 \\
0.0747 \\
0.0848 \\
0.0948 \\
0.1740 \\
0.2101 \\
0.3675 \\
0.4371 \\
0.6161\end{array}$ & $\begin{array}{l}0.02 \\
0.03 \\
0.04 \\
0.05 \\
0.06 \\
0.07 \\
0.08 \\
0.09 \\
0.10 \\
0.20 \\
0.30 \\
0.40 \\
0.55\end{array}$ & $\begin{array}{l}0.02 \\
0.03 \\
0.04 \\
0.05 \\
0.06 \\
0.07 \\
0.08 \\
0.09 \\
0.10 \\
0.20 \\
0.30 \\
0.40 \\
0.55 \\
0.75\end{array}$ & $\begin{array}{l}0.000 E+00 \\
2.180 E+06 \\
0.000 E+00 \\
0.000 E+00 \\
0.000 E+00 \\
0.000 E+00 \\
0.000 E+00 \\
0.000 E+00 \\
0.000 E+00 \\
3.255 E+05 \\
3.108 E+04 \\
8.480 E+04 \\
1.784 E+06 \\
1.595 E+06\end{array}$ & $\begin{array}{l}1.097 \mathrm{E}+04 \\
4.966 \mathrm{E}+03 \\
2.783 \mathrm{E}+03 \\
1.540 \mathrm{E}+03 \\
9.858 \mathrm{E}+02 \\
7.180 \mathrm{E}+02 \\
5.053 \mathrm{E}+02 \\
3.754 \mathrm{E}+02 \\
2.921 \mathrm{E}+02 \\
1.001 \mathrm{E}+03 \\
1.377 \mathrm{E}+02 \\
2.437 \mathrm{E}+01 \\
3.736 \mathrm{E}+00 \\
3.994 \mathrm{E}-02\end{array}$ & $\begin{array}{l}1.097 \mathrm{E}+04 \\
2.185 \mathrm{E}+06 \\
2.783 \mathrm{E}+03 \\
1.540 \mathrm{E}+03 \\
9.858 \mathrm{E}+02 \\
7.180 \mathrm{E}+02 \\
5.053 \mathrm{E}+02 \\
3.754 \mathrm{E}+02 \\
2.921 \mathrm{E}+02 \\
3.265 \mathrm{E}+05 \\
3.122 \mathrm{E}+04 \\
8.483 \mathrm{E}+04 \\
1.784 \mathrm{E}+06 \\
1.595 \mathrm{E}+06\end{array}$ \\
\hline
\end{tabular}

Total Photons/sec: $\quad 6.000 \mathrm{E}+06 \quad 2.430 \mathrm{E}+04 \quad 6.025 \mathrm{E}+06$

\begin{tabular}{|c|c|c|c|c|c|c|}
\hline $\begin{array}{l}\gg \text { CS } \\
\text { Group } \\
\text { No. }\end{array}$ & $\begin{array}{l}134 \\
\text { Photon } \\
\text { Mean }\end{array}$ & $\begin{array}{l}=55) \\
\text { Energy, } \\
\text { Low }\end{array}$ & $\begin{array}{l}\mathrm{MeV} \\
\mathrm{High}\end{array}$ & $\begin{array}{l}\text { eight }(319)= \\
\text { Photon Prod } \\
\text { Gamma\&Xray }\end{array}$ & $\begin{array}{c}1.930 E+01 \\
\text { iction Rate } \\
\text { Bremss. }\end{array}$ & $\begin{array}{c}\text { Total } \\
\text { photon/s }\end{array}$ \\
\hline 1 & 0.0142 & 0.01 & 0.02 & $0.000 \mathrm{E}+00$ & $3.383 E+03$ & $3.383 E+03$ \\
\hline 2 & 0.0286 & 0.02 & 0.03 & $5.998 E+03$ & $1.948 E+03$ & $7.946 E+03$ \\
\hline 3 & 0.0346 & 0.03 & 0.04 & $0.000 \mathrm{E}+00$ & $1.333 E+03$ & 1. $333 E+03$ \\
\hline 4 & 0.0444 & 0.04 & 0.05 & $0.000 E+00$ & $7.481 E+02$ & $7.481 E+02$ \\
\hline 5 & 0.0548 & 0.05 & 0.06 & $0.000 \mathrm{E}+00$ & $4.366 \mathrm{E}+02$ & $4.366 \mathrm{E}+02$ \\
\hline 6 & 0.0648 & 0.06 & 0.07 & $0.000 E+00$ & $3.477 E+02$ & $3.477 E+02$ \\
\hline 7 & 0.0747 & 0.07 & 0.08 & $0.000 \mathrm{E}+00$ & $2.600 \mathrm{E}+02$ & $2.600 \mathrm{E}+02$ \\
\hline 8 & 0.0848 & 0.08 & 0.09 & $0.000 E+00$ & $1.910 \mathrm{E}+02$ & $1.910 \mathrm{E}+02$ \\
\hline 9 & 0.0948 & 0.09 & 0.10 & $0.000 E+00$ & $1.585 \mathrm{E}+02$ & $1.585 E+02$ \\
\hline 10 & 0.1343 & 0.10 & 0.20 & $0.000 E+00$ & $6.324 E+02$ & +02 \\
\hline 11 & 0.2372 & 0.20 & 0.30 & $0.000 \mathrm{E}+00$ & 1. $232 \mathrm{E}+02$ & $2 \mathrm{E}+02$ \\
\hline 12 & 0.3369 & 0.30 & 0.40 & $0.000 E+00$ & $2.700 \mathrm{E}+01$ & $2.700 E+01$ \\
\hline 13 & 0.4754 & 0.40 & 0.55 & $1.043 E+04$ & $5.312 \mathrm{E}+00$ & $1.043 E+04$ \\
\hline 14 & 0.5973 & 0.55 & 0.75 & $8.670 E+05$ & $1.797 \mathrm{E}-01$ & $8.670 E+05$ \\
\hline 15 & 0.7964 & 0.75 & 0.90 & $6.722 E+05$ & $4.976 \mathrm{E}-04$ & $6.722 E+05$ \\
\hline 1.6 & 0.9000 & 0.90 & 1.10 & $0.000 E+00$ & $7.385 E-06$ & 7. $385 \mathrm{E}-06$ \\
\hline 1.7 & 1.1220 & 1.10 & 1.35 & $1.999 \mathrm{E}+04$ & $0.000 \mathrm{E}+00$ & $1.999 \mathrm{E}+04$ \\
\hline 1.8 & 1.3650 & 1.35 & 1.60 & $2.171 E+04$ & $0.000 \mathrm{E}+00$ & $2.171 E+04$ \\
\hline
\end{tabular}

Total Photons/sec: $\quad 1.597 \mathrm{E}+06 \quad 9.594 \mathrm{E}+03 \quad 1.607 \mathrm{E}+06$ 
HNF-SD-WM-CN-091

Rev. 0

Page 13 of 64

\begin{tabular}{ccccccc}
$\gg>C S-137$ & $(Z=55)$ & \multicolumn{4}{c}{ Weight $(335)=8.450 \mathrm{E}+02 \mu C \mathrm{C}$} \\
$\begin{array}{c}\text { Group } \\
\text { No. }\end{array}$ & $\begin{array}{c}\text { Photon } \\
\text { Mean }\end{array}$ & $\begin{array}{c}\text { Energy, } \\
\text { Low }\end{array}$ & $\begin{array}{c}\text { MeV } \\
\text { High }\end{array}$ & $\begin{array}{c}\text { Photon Production Rate } \\
\text { Gamma\&Xray }\end{array}$ & $\begin{array}{c}\text { Total } \\
\text { Bremss. }\end{array}$ & photon $/ \mathrm{s}$ \\
\hline 1 & 0.0143 & 0.01 & 0.02 & $0.000 \mathrm{E}+00$ & $1.706 \mathrm{E}+05$ & $1.706 \mathrm{E}+05$ \\
2 & 0.0246 & 0.02 & 0.03 & $0.000 \mathrm{E}+00$ & $9.901 \mathrm{E}+04$ & $9.901 \mathrm{E}+04$ \\
3 & 0.0344 & 0.03 & 0.04 & $0.000 \mathrm{E}+00$ & $5.260 \mathrm{E}+04$ & $5.260 \mathrm{E}+04$ \\
4 & 0.0448 & 0.04 & 0.05 & $0.000 \mathrm{E}+00$ & $3.119 \mathrm{E}+04$ & $3.119 \mathrm{E}+04$ \\
5 & 0.0547 & 0.05 & 0.06 & $0.000 \mathrm{E}+00$ & $2.351 \mathrm{E}+04$ & $2.351 \mathrm{E}+04$ \\
6 & 0.0647 & 0.06 & 0.07 & $0.000 \mathrm{E}+00$ & $1.643 \mathrm{E}+04$ & $1.643 \mathrm{E}+04$ \\
7 & 0.0748 & 0.07 & 0.08 & $0.000 \mathrm{E}+00$ & $1.261 \mathrm{E}+04$ & $1.261 \mathrm{E}+04$ \\
8 & 0.0847 & 0.08 & 0.09 & $0.000 \mathrm{E}+00$ & $9.109 \mathrm{E}+03$ & $9.109 \mathrm{E}+03$ \\
9 & 0.0949 & 0.09 & 0.10 & $0.000 \mathrm{E}+00$ & $6.934 \mathrm{E}+03$ & $6.934 \mathrm{E}+03$ \\
10 & 0.1340 & 0.10 & 0.20 & $0.000 \mathrm{E}+00$ & $2.776 \mathrm{E}+04$ & $2.776 \mathrm{E}+04$ \\
11 & 0.2379 & 0.20 & 0.30 & $0.000 \mathrm{E}+00$ & $5.305 \mathrm{E}+03$ & $5.305 \mathrm{E}+03$ \\
12 & 0.3400 & 0.30 & 0.40 & $0.000 \mathrm{E}+00$ & $1.393 \mathrm{E}+03$ & $1.393 \mathrm{E}+03$ \\
13 & 0.4580 & 0.40 & 0.55 & $0.000 \mathrm{E}+00$ & $6.258 \mathrm{E}+02$ & $6.258 \mathrm{E}+02$ \\
14 & 0.6219 & 0.55 & 0.75 & $0.000 \mathrm{E}+00$ & $2.013 \mathrm{E}+02$ & $2.013 \mathrm{E}+02$ \\
15 & 0.8048 & 0.75 & 0.90 & $0.000 \mathrm{E}+00$ & $2.736 \mathrm{E}+01$ & $2.736 \mathrm{E}+01$ \\
16 & 0.9459 & 0.90 & 1.10 & $0.000 \mathrm{E}+00$ & $3.982 \mathrm{E}+00$ & $3.982 \mathrm{E}+00$ \\
17 & 1.1000 & 1.10 & 1.35 & $0.000 \mathrm{E}+00$ & $2.896 \mathrm{E}-02$ & $2.896 \mathrm{E}-02$ \\
\hline
\end{tabular}

\begin{tabular}{|c|c|c|c|c|c|c|}
\hline $\begin{array}{c}\gg \text { BA } \\
\text { Group } \\
\text { No. }\end{array}$ & $\begin{array}{l}137 \mathrm{M} \\
\text { Photon } \\
\text { Mean }\end{array}$ & $\begin{array}{l}=56) \\
\text { Energy, } \\
\text { Low }\end{array}$ & $\begin{array}{l}\mathrm{MeV} \\
\mathrm{High}\end{array}$ & $\begin{array}{c}\text { Weight }(336) \\
\text { Photon Proc } \\
\text { Gamma\&Xray }\end{array}$ & $\begin{array}{c}7.990 E+02 \\
\text { uction Rate } \\
\text { Bremss. }\end{array}$ & $\begin{array}{l}\text { Total } \\
\text { photon/s }\end{array}$ \\
\hline $\begin{array}{r}3 \\
14\end{array}$ & $\begin{array}{l}0.0329 \\
0.6616\end{array}$ & $\begin{array}{l}0.03 \\
0.55\end{array}$ & $\begin{array}{l}0.04 \\
0.75\end{array}$ & $\begin{array}{l}2.152 E+06 \\
2.660 E+07\end{array}$ & $\begin{array}{l}0.000 E+00 \\
0.000 E+00\end{array}$ & $\begin{array}{l}2.152 E+06 \\
2.660 E+07\end{array}$ \\
\hline
\end{tabular}

Total Photons/sec: $2.875 \mathrm{E}+07 \quad 0.000 \mathrm{E}+00 \quad 2.875 \mathrm{E}+07$ 
HNF-SD-WM-CN-091

Rev. 0

Page 14 of 64

\begin{tabular}{|c|c|c|c|c|c|c|}
\hline CE- & 144 & 58) & & ight (376) & $120 E+02$ & \\
\hline $\begin{array}{l}\text { Group } \\
\text { No. }\end{array}$ & $\begin{array}{l}\text { Photón } \\
\text { Mean }\end{array}$ & $\begin{array}{l}\text { Energy } \\
\text { Low }\end{array}$ & $\begin{array}{l}\text { MeV } \\
\text { High }\end{array}$ & $\begin{array}{l}\text { Photon Pro } \\
\text { Gamma\&Xray }\end{array}$ & $\begin{array}{c}\text { uction Rate } \\
\text { Bremss. }\end{array}$ & $\begin{array}{l}\text { Total } \\
\text { photon } / \mathrm{s}\end{array}$ \\
\hline 1 & 0.0143 & 0.01 & 0.02 & $0.000 E+00$ & $6.951 \mathrm{E}+04$ & $6.951 E+04$ \\
\hline 2 & 0.0241 & 0.02 & 0.03 & $0.000 E+00$ & $2.382 \mathrm{E}+04$ & $2.382 \mathrm{E}+04$ \\
\hline 3 & 0.0358 & 0.03 & 0.04 & $1.899 E+06$ & $1.267 \mathrm{E}+04$ & $1.912 \mathrm{E}+06$ \\
\hline 4 & 0.0409 & 0.04 & 0.05 & $5.427 E+05$ & $7.357 \mathrm{E}+03$ & $5.500 E+05$ \\
\hline 5 & 0.0546 & 0.05 & 0.06 & $0.000 E+00$ & $4.675 \mathrm{E}+03$ & $4.675 \mathrm{E}+03$ \\
\hline 6 & 0.0647 & 0.06 & 0.07 & $0.000 E+00$ & $3.197 \mathrm{E}+03$ & $3.197 E+03$ \\
\hline 7 & 0.0747 & 0.07 & 0.08 & $0.000 E+00$ & $2.177 \mathrm{E}+03$ & $2.177 E+03$ \\
\hline 8 & 0.0801 & 0.08 & 0.09 & $4.215 E+05$ & $1.533 E+03$ & $4.230 E+05$ \\
\hline 9 & 0.0947 & 0.09 & 0.10 & $0.000 E+00$ & $1.097 \mathrm{E}+03$ & $1.097 E+03$ \\
\hline $1: 0$ & 0.1335 & 0.10 & 0.20 & $2.845 E+06$ & $2.792 E+03$ & $2.848 E+06$ \\
\hline ]: 1 & 0.21 & 0.20 & 0.30 & $000 E+00$ & $6.976 E+01$ & $6.976 E+01$ \\
\hline$\llbracket 2$ & 0.3 & 0.30 & 0.40 & $0.000 E+00$ & $2.692 \mathrm{E}-02$ & $2.692 \mathrm{E}-02$ \\
\hline
\end{tabular}

Total Photons/sec: $\quad 5.709 E+06 \quad 1.289 E+05 \quad 5.838 E+06$

$\gg$ PR-144 (Z = 59) Weight $(377)=7.120 \mathrm{E}+02 \mu \mathrm{Ci}$

Group Photon Energy, MeV Photon Production Rate Total

No. Mean Low High Gamma\&Xray Bremss. photon/s

\begin{tabular}{rllllll}
\hline 1 & 0.0145 & 0.01 & 0.02 & $0.000 \mathrm{E}+00$ & $1.216 \mathrm{E}+06$ & $1.216 \mathrm{E}+06$ \\
2 & 0.0246 & 0.02 & 0.03 & $0.000 \mathrm{E}+00$ & $6.079 \mathrm{E}+05$ & $6.079 \mathrm{E}+05$ \\
3 & 0.0346 & 0.03 & 0.04 & $0.000 \mathrm{E}+00$ & $4.095 \mathrm{E}+05$ & $4.095 \mathrm{E}+05$ \\
4 & 0.0448 & 0.04 & 0.05 & $0.000 \mathrm{E}+00$ & $2.830 \mathrm{E}+05$ & $2.830 \mathrm{E}+05$ \\
5 & 0.0548 & 0.05 & 0.06 & $0.000 \mathrm{E}+00$ & $2.228 \mathrm{E}+05$ & $2.228 \mathrm{E}+05$ \\
6 & 0.0648 & 0.06 & 0.07 & $0.000 \mathrm{E}+00$ & $1.697 \mathrm{E}+05$ & $1.697 \mathrm{E}+05$ \\
7 & 0.0749 & 0.07 & 0.08 & $0.000 \mathrm{E}+00$ & $1.359 \mathrm{E}+05$ & $1.359 \mathrm{E}+05$ \\
8 & 0.0850 & 0.08 & 0.09 & $0.000 \mathrm{E}+00$ & $1.264 \mathrm{E}+05$ & $1.264 \mathrm{E}+05$ \\
9 & 0.0949 & 0.09 & 0.10 & $0.000 \mathrm{E}+00$ & $1.191 \mathrm{E}+05$ & $1.191 \mathrm{E}+05$ \\
110 & 0.1424 & 0.10 & 0.20 & $0.000 \mathrm{E}+00$ & $7.941 \mathrm{E}+05$ & $7.941 \mathrm{E}+05$ \\
11 & 0.2418 & 0.20 & 0.30 & $0.000 \mathrm{E}+00$ & $2.581 \mathrm{E}+05$ & $2.581 \mathrm{E}+05$ \\
112 & 0.3447 & 0.30 & 0.40 & $0.000 \mathrm{E}+00$ & $1.329 \mathrm{E}+05$ & $1.329 \mathrm{E}+05$ \\
11 & 0.4668 & 0.40 & 0.55 & $0.000 \mathrm{E}+00$ & $1.066 \mathrm{E}+05$ & $1.066 \mathrm{E}+05$ \\
114 & 0.6876 & 0.55 & 0.75 & $3.899 \mathrm{E}+05$ & $6.955 \mathrm{E}+04$ & $4.594 \mathrm{E}+05$ \\
15 & 0.8186 & 0.75 & 0.90 & $0.000 \mathrm{E}+00$ & $2.772 \mathrm{E}+04$ & $2.772 \mathrm{E}+04$ \\
16 & 0.9894 & 0.90 & 1.10 & $0.000 \mathrm{E}+00$ & $2.153 \mathrm{E}+04$ & $2.153 \mathrm{E}+04$ \\
17 & 1.2095 & 1.10 & 1.35 & $0.000 \mathrm{E}+00$ & $1.377 \mathrm{E}+04$ & $1.377 \mathrm{E}+04$ \\
18 & 1.4867 & 1.35 & 1.60 & $7.903 \mathrm{E}+04$ & $6.443 \mathrm{E}+03$ & $8.547 \mathrm{E}+04$ \\
19 & 1.6891 & 1.60 & 1.80 & $0.000 \mathrm{E}+00$ & $2.472 \mathrm{E}+03$ & $2.472 \mathrm{E}+03$ \\
20 & 1.8874 & 1.80 & 2.00 & $0.000 \mathrm{E}+00$ & $1.227 \mathrm{E}+03$ & $1.227 \mathrm{E}+03$ \\
21 & 2.1857 & 2.00 & 2.20 & $2.028 \mathrm{E}+05$ & $5.577 \mathrm{E}+02$ & $2.034 \mathrm{E}+05$ \\
22 & 2.2828 & 2.20 & 2.40 & $0.000 \mathrm{E}+00$ & $2.135 \mathrm{E}+02$ & $2.135 \mathrm{E}+02$ \\
23 & 2.4759 & 2.40 & 2.60 & $0.000 \mathrm{E}+00$ & $6.414 \mathrm{E}+01$ & $6.414 \mathrm{E}+01$ \\
24 & 2.6672 & 2.60 & 2.80 & $0.000 \mathrm{E}+00$ & $1.240 \mathrm{E}+01$ & $1.240 \mathrm{E}+01$ \\
25 & 2.8000 & 2.80 & 3.20 & $0.000 \mathrm{E}+00$ & $1.268 \mathrm{E}+00$ & $1.268 \mathrm{E}+00$ \\
\hline 1
\end{tabular}

Total Photons/sec: $\quad 6.718 \mathrm{E}+05 \quad 4.726 \mathrm{E}+06 \quad 5.398 \mathrm{E}+06$ 
HNF-SD-WM-CN-091

Rev. 0

Page 15 of 64

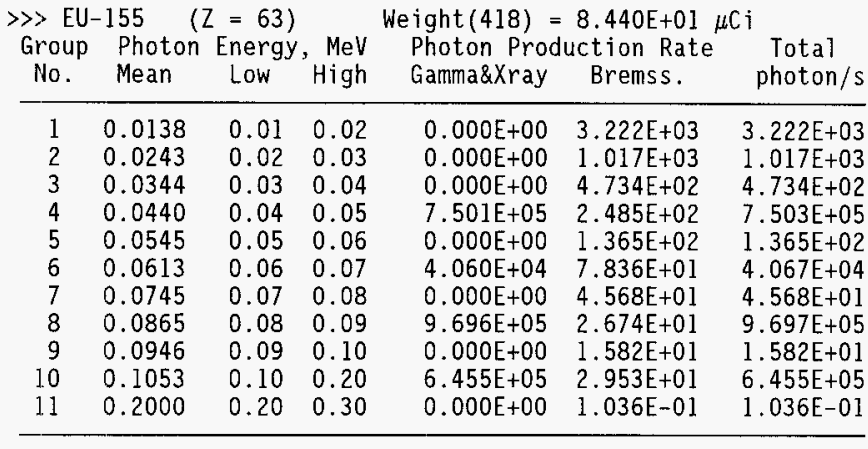

Total Photons/sec: $\quad 2,406 \mathrm{E}+06 \quad 5.294 \mathrm{E}+03 \quad 2.411 \mathrm{E}+06$

\begin{tabular}{|c|c|c|c|c|c|c|}
\hline $\begin{array}{l}\text { Qroup } \\
\text { Group } \\
\text { No. }\end{array}$ & $\begin{array}{c}-234 \\
\text { Photon } \\
\text { Mean }\end{array}$ & $\begin{array}{l}=92) \\
\text { inergy, } \\
\text { Low }\end{array}$ & $\begin{array}{l}\text { MeV } \\
\text { High }\end{array}$ & $\begin{array}{l}\text { ight }(520)= \\
\text { Photon Proc } \\
\text { Gamma\&Xray }\end{array}$ & $\begin{array}{l}.720 \mathrm{E}-06 \\
\text { ction Rate } \\
\text { Bremss. }\end{array}$ & $\begin{array}{c}\text { Total } \\
\text { photon/s }\end{array}$ \\
\hline $\begin{array}{r}1 \\
2 \\
3 \\
4 \\
5 \\
6 \\
7 \\
8 \\
9 \\
10 \\
13 \\
1.4\end{array}$ & $\begin{array}{l}0.0130 \\
0.0231 \\
0.0334 \\
0.0439 \\
0.0532 \\
0.0639 \\
0.0736 \\
0.0830 \\
0.0916 \\
0.1210 \\
0.4900 \\
0.5800\end{array}$ & $\begin{array}{l}0.01 \\
0.02 \\
0.03 \\
0.04 \\
0.05 \\
0.06 \\
0.07 \\
0.08 \\
0.09 \\
0.10 \\
0.40 \\
0.55\end{array}$ & $\begin{array}{l}0.02 \\
0.03 \\
0.04 \\
0.05 \\
0.06 \\
0.07 \\
0.08 \\
0.09 \\
0.10 \\
0.20 \\
0.55 \\
0.75\end{array}$ & $\begin{array}{l}6.682 \mathrm{E}-03 \\
0.000 \mathrm{E}+00 \\
0.000 \mathrm{E}+00 \\
0.000 \mathrm{E}+00 \\
7.573 \mathrm{E}-05 \\
0.000 \mathrm{E}+00 \\
0.000 \mathrm{E}+00 \\
0.000 \mathrm{E}+00 \\
0.000 \mathrm{E}+00 \\
2.546 \mathrm{E}-05 \\
2.546 \mathrm{E}-06 \\
7.637 \mathrm{E}-09\end{array}$ & $\begin{array}{l}5.785 \mathrm{E}-07 \\
5.414 \mathrm{E}-08 \\
6.507 \mathrm{E}-09 \\
1.251 \mathrm{E}-09 \\
3.967 \mathrm{E}-10 \\
1.191 \mathrm{E}-10 \\
2.770 \mathrm{E}-11 \\
3.921 \mathrm{E}-12 \\
1.806 \mathrm{E}-13 \\
6.653 \mathrm{E}-18 \\
0.000 \mathrm{E}+00 \\
0.000 \mathrm{E}+00\end{array}$ & $\begin{array}{l}6.683 \mathrm{E}-03 \\
5.414 \mathrm{E}-08 \\
6.507 \mathrm{E}-09 \\
1.251 \mathrm{E}-09 \\
7.573 \mathrm{E}-05 \\
1.191 \mathrm{E}-10 \\
2.770 \mathrm{E}-11 \\
3.921 \mathrm{E}-12 \\
1.806 \mathrm{E}-13 \\
2.546 \mathrm{E}-05 \\
2.546 \mathrm{E}-06 \\
7.637 \mathrm{E}-09\end{array}$ \\
\hline
\end{tabular}

Total Photons/sec: $6.786 \mathrm{E}-03 \quad 6.409 \mathrm{E}-07 \quad 6.787 \mathrm{E}-03$ 
HNF-SD-WM-CN-091

Rev. 0

Page 16 of 64

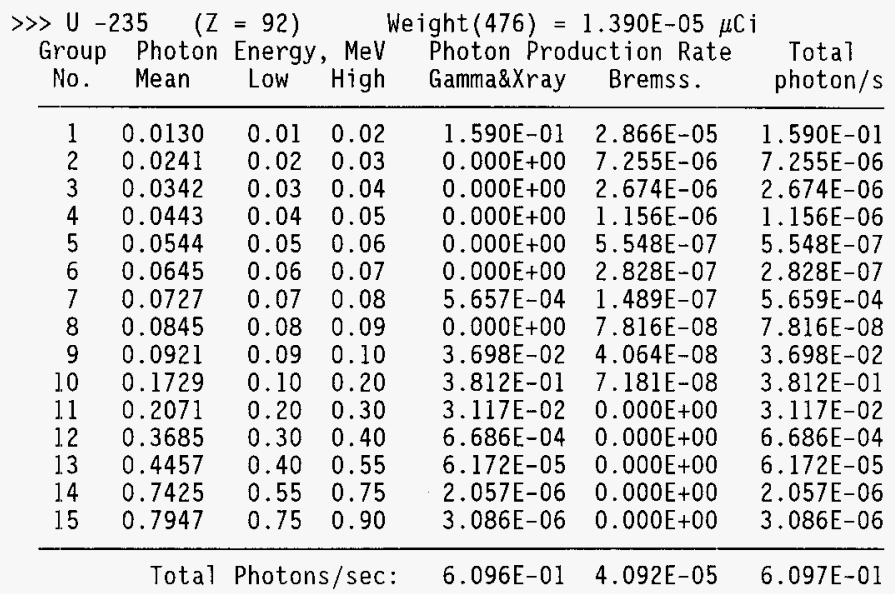

\begin{tabular}{|c|c|c|c|c|c|c|}
\hline $\begin{array}{l}\gg \gg U \\
\text { Group } \\
\text { No. }\end{array}$ & $\begin{array}{c}-236 \\
\text { Photon } \\
\text { Mean }\end{array}$ & $\begin{array}{l}=92 \text { ) } \\
\text { Energy } \\
\text { Low }\end{array}$ & $\begin{array}{r}\text { MeV } \\
\text { High }\end{array}$ & $\begin{array}{l}\text { eight ( } 398) \\
\text { Photon Proo } \\
\text { Gamma\&Xray }\end{array}$ & $\begin{array}{c}4.970 \mathrm{E}-05 \\
\text { iction Rate } \\
\text { Bremss. }\end{array}$ & $\begin{array}{c}\text { Tota } 7 \\
\text { photon/s }\end{array}$ \\
\hline $\begin{array}{l}1 \\
2 \\
3 \\
4 \\
5 \\
6 \\
7 \\
8 \\
9\end{array}$ & $\begin{array}{l}0.0128 \\
0.0230 \\
0.0335 \\
0.0440 \\
0.0539 \\
0.0682 \\
0.0731 \\
0.0817 \\
0.0900\end{array}$ & $\begin{array}{l}0.01 \\
0.02 \\
0.03 \\
0.04 \\
0.05 \\
0.06 \\
0.07 \\
0.08 \\
0.09\end{array}$ & $\begin{array}{l}0.02 \\
0.03 \\
0.04 \\
0.05 \\
0.06 \\
0.07 \\
0.08 \\
0.09 \\
0.10\end{array}$ & $\begin{array}{l}0.000 E+00 \\
0.000 E+00 \\
0.000 E+00 \\
0.000 E+00 \\
0.000 E+00 \\
2.023 E-03 \\
0.000 E+00 \\
0.000 E+00 \\
0.000 E+00\end{array}$ & $\begin{array}{l}1.075 \mathrm{E}-05 \\
8.918 \mathrm{E}-07 \\
1.046 \mathrm{E}-07 \\
2.557 \mathrm{E}-08 \\
7.627 \mathrm{E}-09 \\
1.828 \mathrm{E}-09 \\
2.799 \mathrm{E}-10 \\
1.487 \mathrm{E}-11 \\
7.756 \mathrm{E}-15\end{array}$ & $\begin{array}{l}1.075 \mathrm{E}-05 \\
8.918 \mathrm{E}-07 \\
1.046 \mathrm{E}-07 \\
2.557 \mathrm{E}-08 \\
7.627 \mathrm{E}-09 \\
2.023 \mathrm{E}-03 \\
2.799 \mathrm{E}-10 \\
1.487 \mathrm{E}-11 \\
7.756 \mathrm{E}-15\end{array}$ \\
\hline
\end{tabular}

Total Photons/sec: $2.023 \mathrm{E}-03 \quad 1.179 \mathrm{E}-05 \quad 2.035 \mathrm{E}-03$ 
HNF-SD-WM-CN-091

Rev. 0

Page 17 of 64

\begin{tabular}{|c|c|c|c|c|c|c|}
\hline $\begin{array}{l}>>\text { U - } \\
\text { Group } \\
\text { No. }\end{array}$ & $\begin{array}{l}238 \quad(Z \\
\text { Photon } \\
\text { Mean }\end{array}$ & $\begin{array}{l}=92 \text { ) } \\
\text { Energy, } \\
\text { Low }\end{array}$ & , MeV & $\begin{array}{l}\text { ight }(526)= \\
\text { Photon Pro } \\
\text { Gamma\&Xray }\end{array}$ & $\begin{array}{c}\text { 8. } 310 \mathrm{E}-03 \mu \mathrm{C} \\
\text { uction Rate } \\
\text { Bremss. }\end{array}$ & $\begin{array}{l}\text { Total } \\
\text { photon/s }\end{array}$ \\
\hline $\begin{array}{l}1 \\
2 \\
3 \\
4 \\
5 \\
6 \\
7 \\
8\end{array}$ & $\begin{array}{l}0.0130 \\
0.0230 \\
0.0335 \\
0.0440 \\
0.0538 \\
0.0664 \\
0.0729 \\
0.0808\end{array}$ & $\begin{array}{l}0.01 \\
0.02 \\
0.03 \\
0.04 \\
0.05 \\
0.06 \\
0.07 \\
0.08\end{array}$ & $\begin{array}{l}0.02 \\
0.03 \\
0.04 \\
0.05 \\
0.06 \\
0.07 \\
0.08 \\
0.09\end{array}$ & $\begin{array}{l}2.715 \mathrm{E}+01 \\
0.000 \mathrm{E}+00 \\
0.000 \mathrm{E}+00 \\
0.000 \mathrm{E}+00 \\
0.000 \mathrm{E}+00 \\
3.075 \mathrm{E}-01 \\
0.000 \mathrm{E}+00 \\
0.000 \mathrm{E}+00\end{array}$ & $\begin{array}{l}1.617 \mathrm{E}-03 \\
1.339 \mathrm{E}-04 \\
1.523 \mathrm{E}-05 \\
3.472 \mathrm{E}-06 \\
9.529 \mathrm{E}-07 \\
1.968 \mathrm{E}-07 \\
2.496 \mathrm{E}-08 \\
1.207 \mathrm{E}-09\end{array}$ & $\begin{array}{l}2.715 \mathrm{E}+01 \\
1.339 \mathrm{E}-04 \\
1.523 \mathrm{E}-05 \\
3.472 \mathrm{E}-06 \\
9.529 \mathrm{E}-07 \\
3.075 \mathrm{E}-01 \\
2.496 \mathrm{E}-08 \\
1.207 \mathrm{E}-09\end{array}$ \\
\hline
\end{tabular}

\begin{tabular}{|c|c|c|c|c|c|c|}
\hline $\begin{array}{l}\gg A M \\
\text { Group } \\
\text { No. }\end{array}$ & $\begin{array}{l}241 \\
\text { Photon } \\
\text { Mean }\end{array}$ & $\begin{array}{l}=95) \\
\text { Energy } \\
\text { Low }\end{array}$ & $\begin{array}{l}\text { MeV } \\
\mathrm{High}\end{array}$ & $\begin{array}{l}\text { eight }(496)= \\
\text { Photon Proo } \\
\text { Gamma\&Xray }\end{array}$ & $\begin{array}{l}\text { Bion Rat } \\
\text { Bremss. }\end{array}$ & $\begin{array}{c}\text { Total } \\
\text { photon/s }\end{array}$ \\
\hline $\begin{array}{r}1 \\
2 \\
3 \\
4 \\
5 \\
6 \\
7 \\
8 \\
9 \\
10 \\
11 \\
12 \\
13 \\
14 \\
15\end{array}$ & $\begin{array}{l}32 \\
30 \\
95 \\
99 \\
32 \\
21 \\
89 \\
87 \\
32 \\
41 \\
48 \\
58\end{array}$ & $\begin{array}{l}0.03 \\
0.04 \\
0.05 \\
0.06 \\
0.07 \\
0.08 \\
0.09 \\
0.10 \\
0.20 \\
0.30 \\
0.40 \\
0.55 \\
0.75\end{array}$ & $\begin{array}{l}0.02 \\
0.03 \\
0.04 \\
0.05 \\
0.06 \\
0.07 \\
0.08 \\
0.09 \\
0.10 \\
0.20 \\
0.30 \\
0.40 \\
0.55 \\
0.75 \\
0.90\end{array}$ & $\begin{array}{l}8.7 \\
7.1 \\
2.8 \\
1.5 \\
0.0 \\
0.0 \\
1.6 \\
2.2 \\
6.8 \\
1.0 \\
6.2 \\
5.4 \\
1.7\end{array}$ & $\begin{array}{l}2.050 \mathrm{E}-01 \\
3.238 \mathrm{E}-02 \\
6.396 \mathrm{E}-03 \\
9.423 \mathrm{E}-04 \\
6.034 \mathrm{E}-05 \\
4.201 \mathrm{E}-06 \\
0.000 \mathrm{E}+00 \\
0.000 \mathrm{E}+00 \\
0.000 \mathrm{E}+00 \\
0.000 \mathrm{E}+00 \\
0.000 \mathrm{E}+00 \\
0.000 \mathrm{E}+00\end{array}$ & $\begin{array}{l}+05 \\
+04 \\
+02 \\
+02 \\
+05 \\
+01 \\
-05 \\
-06 \\
+02 \\
+02 \\
+00 \\
+01 \\
-01 \\
+00 \\
-01\end{array}$ \\
\hline
\end{tabular}

Total Photons/sec: $6.472 \mathrm{E}+05 \quad 1.856 \mathrm{E}+01 \quad 6.472 \mathrm{E}+05$ 
HNF-SD-WM-CN-091

Rev. 0

Page 18 of 64

Organic Tank (H0-64-4278) Line Source - Middle

Shield Composition, g/cc

Shield 1 Shield 2 Shield 3

\begin{tabular}{llll}
\hline AIR & $0.000 \mathrm{E}+00$ & $0.000 \mathrm{E}+00$ & $1.290 \mathrm{E}-03$ \\
ALUMINUM & $8.122 \mathrm{E}-01$ & $0.000 \mathrm{E}+00$ & $0.000 \mathrm{E}+00$ \\
IRON & $0.000 \mathrm{E}+00$ & $7.800 \mathrm{E}+00$ & $0.000 \mathrm{E}+00$ \\
\hline Totals: & $8.122 \mathrm{E}-01$ & $7.800 \mathrm{E}+00$ & $1.290 \mathrm{E}-03$ \\
\hline
\end{tabular}

E, MeV Linear Attenuation, per $\mathrm{cm}$ (last region is air)

\begin{tabular}{llll}
\hline 0.0145 & $7.175 \mathrm{E}+00$ & $5.015 \mathrm{E}+02$ & $2.205 \mathrm{E}-03$ \\
0.0254 & $1.430 \mathrm{E}+00$ & $1.038 \mathrm{E}+02$ & $5.985 \mathrm{E}-04$ \\
0.0345 & $6.386 \mathrm{E}-01$ & $4.319 \mathrm{E}+01$ & $3.741 \mathrm{E}-04$ \\
0.0442 & $3.703 \mathrm{E}-01$ & $2.131 \mathrm{E}+01$ & $2.891 \mathrm{E}-04$ \\
0.0553 & $2.476 \mathrm{E}-01$ & $1.155 \mathrm{E}+01$ & $2.486 \mathrm{E}-04$ \\
0.0648 & $1.992 \mathrm{E}-01$ & $7.729 \mathrm{E}+00$ & $2.318 \mathrm{E}-04$ \\
0.0748 & $1.714 \mathrm{E}-01$ & $5.439 \mathrm{E}+00$ & $2.186 \mathrm{E}-04$ \\
0.0847 & $1.543 \mathrm{E}-01$ & $4.095 \mathrm{E}+00$ & $2.092 \mathrm{E}-04$ \\
0.0950 & $1.419 \mathrm{E}-01$ & $3.215 \mathrm{E}+00$ & $2.019 \mathrm{E}-04$ \\
0.1406 & $1.159 \mathrm{E}-01$ & $1.698 \mathrm{E}+00$ & $1.793 \mathrm{E}-04$ \\
0.2408 & $9.207 \mathrm{E}-02$ & $9.921 \mathrm{E}-01$ & $1.496 \mathrm{E}-04$ \\
0.3468 & $7.972 \mathrm{E}-02$ & $7.904 \mathrm{E}-01$ & $1.302 \mathrm{E}-04$ \\
0.5081 & $6.816 \mathrm{E}-02$ & $6.501 \mathrm{E}-01$ & $1.113 \mathrm{E}-04$ \\
0.6409 & $6.148 \mathrm{E}-02$ & $5.808 \mathrm{E}-01$ & $1.007 \mathrm{E}-04$ \\
0.8129 & $5.512 \mathrm{E}-02$ & $5.170 \mathrm{E}-01$ & $9.044 \mathrm{E}-05$ \\
1.0457 & $4.876 \mathrm{E}-02$ & $4.559 \mathrm{E}-01$ & $8.012 \mathrm{E}-05$ \\
1.2414 & $4.473 \mathrm{E}-02$ & $4.178 \mathrm{E}-01$ & $7.347 \mathrm{E}-05$ \\
1.4077 & $4.195 \mathrm{E}-02$ & $3.922 \mathrm{E}-01$ & $6.883 \mathrm{E}-05$ \\
1.6910 & $3.820 \mathrm{E}-02$ & $3.584 \mathrm{E}-01$ & $6.266 \mathrm{E}-05$ \\
1.8906 & $3.610 \mathrm{E}-02$ & $3.402 \mathrm{E}-01$ & $5.905 \mathrm{E}-05$ \\
2.1785 & $3.362 \mathrm{E}-02$ & $3.192 \mathrm{E}-01$ & $5.441 \mathrm{E}-05$ \\
2.2890 & $3.281 \mathrm{E}-02$ & $3.123 \mathrm{E}-01$ & $5.279 \mathrm{E}-05$ \\
2.4878 & $3.149 \mathrm{E}-02$ & $3.016 \mathrm{E}-01$ & $5.018 \mathrm{E}-05$ \\
2.6854 & $3.034 \mathrm{E}-02$ & $2.926 \mathrm{E}-01$ & $4.794 \mathrm{E}-05$ \\
2.9224 & $2.912 \mathrm{E}-02$ & $2.835 \mathrm{E}-01$ & $4.576 \mathrm{E}-05$ \\
3.2000 & $2.789 \mathrm{E}-02$ & $2.751 \mathrm{E}-01$ & $4.383 \mathrm{E}-05$ \\
\hline
\end{tabular}


HNF-SD-WM-CN-091

Rev. 0

Page 19 of 64

Organic Tank (HO-64-4278) Line Source - Middle

Source Shields Distance to Detector, $X=2.500 \mathrm{E}+00 \mathrm{~cm}$

Cylindrical Cylindrical Source Volume $=3.255 \mathrm{E}+03 \mathrm{cc}$

Source Mass $=2.643 \mathrm{E}+03$ grams

Source Length $=1.036 \mathrm{E}+03 \mathrm{~cm} \quad$ Distance Along Cylinder, $Y=5.180 \mathrm{E}+02 \mathrm{~cm}$

Integration Specs: NTHETA $=11$ NPSI $=11$ DELR computed internally

Total Intervals: $7.623 \mathrm{E}+04$ (photon source is the lst region)

Shield Thickness: $1.000 \mathrm{E}+00,1.270 \mathrm{E}+00,2.300 \mathrm{E}-01 \mathrm{~cm}$

Distances from Dose Point to the Outside of

(1) Source Region: $1.500 \mathrm{E}+00 \mathrm{~cm}$ (2) Next Layer: $2.300 \mathrm{E}-01 \mathrm{~cm}$

Dose Buildup Data for Shield 2 with Effective Atomic Number 26.00

Buildup Material is Iron

Source values are interpreted as $\mu \mathrm{C} i$

Source Scale Factor was $1.000 E+03$

Fluence-to-Dose Conversion: Photons in Air

\begin{tabular}{rcccc}
$\begin{array}{c}\text { Average } \\
\text { E, MeV }\end{array}$ & $\begin{array}{c}\text { Source Total } \\
\text { photons } / \mathrm{sec}\end{array}$ & $\begin{array}{c}\text { Fluence to } \\
\text { Dose Factor }\end{array}$ & $\begin{array}{c}\text { Energy Fluence } \\
\mathrm{MeV} / \mathrm{cm}^{2} / \mathrm{s}\end{array}$ & $\begin{array}{c}\text { Dose Rate } \\
\text { R/hr }\end{array}$ \\
\hline 0.0145 & $1.490 \mathrm{E}+07$ & $9.036 \mathrm{E}-05$ & $0.000 \mathrm{E}+00$ & $0.000 \mathrm{E}+00$ \\
0.0254 & $9.971 \mathrm{E}+06$ & $1.577 \mathrm{E}-05$ & $0.000 \mathrm{E}+00$ & $0.000 \mathrm{E}+00$ \\
0.0345 & $9.163 \mathrm{E}+06$ & $6.335 \mathrm{E}-06$ & $1.498 \mathrm{E}-24$ & $9.487 \mathrm{E}-30$ \\
0.0442 & $4.916 \mathrm{E}+06$ & $3.359 \mathrm{E}-06$ & $2.873 \mathrm{E}-12$ & $9.652 \mathrm{E}-18$ \\
0.0553 & $3.038 \mathrm{E}+06$ & $2.204 \mathrm{E}-06$ & $1.178 \mathrm{E}-06$ & $2.596 \mathrm{E}-12$ \\
0.0648 & $2.273 \mathrm{E}+06$ & $1.835 \mathrm{E}-06$ & $2.140 \mathrm{E}-04$ & $3.927 \mathrm{E}-10$ \\
0.0748 & $1.785 \mathrm{E}+06$ & $1.666 \mathrm{E}-06$ & $4.922 \mathrm{E}-03$ & $8.202 \mathrm{E}-09$ \\
0.0847 & $2.904 \mathrm{E}+06$ & $1.605 \mathrm{E}-06$ & $6.225 \mathrm{E}-02$ & $9.988 \mathrm{E}-08$ \\
0.0950 & $1.370 \mathrm{E}-06$ & $1.592 \mathrm{E}-06$ & $1.191 \mathrm{E}-01$ & $1.896 \mathrm{E}-07$ \\
0.1406 & $1.353 \mathrm{E}+07$ & $1.708 \mathrm{E}-06$ & $3.207 \mathrm{E}+01$ & $5.479 \mathrm{E}-05$ \\
0.2408 & $3.749 \mathrm{E}+06$ & $1.919 \mathrm{E}-06$ & $3.309 \mathrm{E}+01$ & $6.350 \mathrm{E}-05$ \\
0.3468 & $1.906 \mathrm{E}+06$ & $2.017 \mathrm{E}-06$ & $2.997 \mathrm{E}+01$ & $6.044 \mathrm{E}-05$ \\
0.5081 & $5.572 \mathrm{E}+07$ & $2.040 \mathrm{E}-06$ & $1.474 \mathrm{E}+03$ & $3.008 \mathrm{E}-03$ \\
0.6409 & $5.733 \mathrm{E}+07$ & $2.030 \mathrm{E}-06$ & $2.040 \mathrm{E}+03$ & $4.141 \mathrm{E}-03$ \\
0.8129 & $3.255 \mathrm{E}+06$ & $1.986 \mathrm{E}-06$ & $1.548 \mathrm{E}+02$ & $3.075 \mathrm{E}-04$ \\
1.0457 & $4.703 \mathrm{E}+06$ & $1.907 \mathrm{E}-06$ & $2.996 \mathrm{E}+02$ & $5.713 \mathrm{E}-04$ \\
1.2414 & $1.199 \mathrm{E}+07$ & $1.845 \mathrm{E}-06$ & $9.245 \mathrm{E}+02$ & $1.705 \mathrm{E}-03$ \\
1.4077 & $2.088 \mathrm{E}+06$ & $1.794 \mathrm{E}-06$ & $1.844 \mathrm{E}+02$ & $3.307 \mathrm{E}-04$ \\
1.6910 & $5.269 \mathrm{E}+04$ & $1.714 \mathrm{E}-06$ & $5.635 \mathrm{E}+00$ & $9.660 \mathrm{E}-06$ \\
1.8906 & $3.066 \mathrm{E}+04$ & $1.662 \mathrm{E}-06$ & $3.677 \mathrm{E}+00$ & $6.112 \mathrm{E}-06$ \\
2.1785 & $2.201 \mathrm{E}+05$ & $1.604 \mathrm{E}-06$ & $3.055 \mathrm{E}+01$ & $4.905 \mathrm{E}-05$ \\
2.2890 & $9.159 \mathrm{E}+03$ & $1.593 \mathrm{E}-06$ & $1.339 \mathrm{E}+00$ & $2.133 \mathrm{E}-06$ \\
2.4878 & $4.520 \mathrm{E}+03$ & $1.537 \mathrm{E}-06$ & $7.184 \mathrm{E}-01$ & $1.104 \mathrm{E}-06$ \\
2.6854 & $1.996 \mathrm{E}+03$ & $1.501 \mathrm{E}-06$ & $3.416 \mathrm{E}-01$ & $5.129 \mathrm{E}-07$ \\
2.9224 & $9.697 \mathrm{E}+02$ & $1.464 \mathrm{E}-06$ & $1.798 \mathrm{E}-01$ & $2.634 \mathrm{E}-07$ \\
3.2000 & $7.528 \mathrm{E}+01$ & $1.425 \mathrm{E}-06$ & $1.522 \mathrm{E}-02$ & $2.169 \mathrm{E}-08$ \\
\hline Totals: & $2.049 \mathrm{E}+08$ photons $/ \mathrm{sec}$ & $5.216 \mathrm{E}+03$ & $1.031 \mathrm{E}-02 \mathrm{R} / \mathrm{hr}$
\end{tabular}


HNF-SD-WM-CN-091

Rev. 0

Page 20 of 64

Organic Tank (H0-64-4278) Annular Source - Middle

Shield Composition, $g / c c$

Shield 1 Shield 2 Shield 3 Shield 4

\begin{tabular}{lllll}
\hline AIR: & $1.290 \mathrm{E}-03$ & $0.000 \mathrm{E}+00$ & $0.000 \mathrm{E}+00$ & $1.290 \mathrm{E}-03$ \\
ALUMINUM & $0.000 \mathrm{E}+00$ & $8.122 \mathrm{E}-01$ & $0.000 \mathrm{E}+00$ & $0.000 \mathrm{E}+00$ \\
IRON & $0.000 \mathrm{E}+00$ & $0.000 \mathrm{E}+00$ & $7.800 \mathrm{E}+00$ & $0.000 \mathrm{E}+00$ \\
\hline Tota1s: & $1.290 \mathrm{E}-03$ & $8.122 \mathrm{E}-01$ & $7.800 \mathrm{E}+00$ & $1.290 \mathrm{E}-03$ \\
\hline E, MeV & Linear Attenuation, per cm (1ast & region is air) \\
\hline 0.0145 & $2.205 \mathrm{E}-03$ & $7.175 \mathrm{E}+00$ & $5.015 \mathrm{E}+02$ & $2.205 \mathrm{E}-03$ \\
0.0254 & $5.985 \mathrm{E}-04$ & $1.430 \mathrm{E}+00$ & $1.038 \mathrm{E}+02$ & $5.985 \mathrm{E}-04$ \\
0.0345 & $3.741 \mathrm{E}-04$ & $6.386 \mathrm{E}-01$ & $4.319 \mathrm{E}+01$ & $3.741 \mathrm{E}-04$ \\
0.0442 & $2.891 \mathrm{E}-04$ & $3.703 \mathrm{E}-01$ & $2.131 \mathrm{E}+01$ & $2.891 \mathrm{E}-04$ \\
0.0553 & $2.486 \mathrm{E}-04$ & $2.476 \mathrm{E}-01$ & $1.155 \mathrm{E}+01$ & $2.486 \mathrm{E}-04$ \\
0.0648 & $2.318 \mathrm{E}-04$ & $1.992 \mathrm{E}-01$ & $7.729 \mathrm{E}+00$ & $2.318 \mathrm{E}-04$ \\
0.0748 & $2.186 \mathrm{E}-04$ & $1.714 \mathrm{E}-01$ & $5.439 \mathrm{E}+00$ & $2.186 \mathrm{E}-04$ \\
0.0847 & $2.092 \mathrm{E}-04$ & $1.543 \mathrm{E}-01$ & $4.095 \mathrm{E}+00$ & $2.092 \mathrm{E}-04$ \\
0.0950 & $2.019 \mathrm{E}-04$ & $1.419 \mathrm{E}-01$ & $3.215 \mathrm{E}+00$ & $2.019 \mathrm{E}-04$ \\
0.1406 & $1.793 \mathrm{E}-04$ & $1.159 \mathrm{E}-01$ & $1.698 \mathrm{E}+00$ & $1.793 \mathrm{E}-04$ \\
0.2408 & $1.496 \mathrm{E}-04$ & $9.207 \mathrm{E}-02$ & $9.921 \mathrm{E}-01$ & $1.496 \mathrm{E}-04$ \\
0.3468 & $1.302 \mathrm{E}-04$ & $7.972 \mathrm{E}-02$ & $7.904 \mathrm{E}-01$ & $1.302 \mathrm{E}-04$ \\
0.5081 & $1.113 \mathrm{E}-04$ & $6.816 \mathrm{E}-02$ & $6.501 \mathrm{E}-01$ & $1.113 \mathrm{E}-04$ \\
0.6409 & $1.007 \mathrm{E}-04$ & $6.148 \mathrm{E}-02$ & $5.808 \mathrm{E}-01$ & $1.007 \mathrm{E}-04$ \\
0.8129 & $9.044 \mathrm{E}-05$ & $5.512 \mathrm{E}-02$ & $5.170 \mathrm{E}-01$ & $9.044 \mathrm{E}-05$ \\
1.0457 & $8.012 \mathrm{E}-05$ & $4.876 \mathrm{E}-02$ & $4.559 \mathrm{E}-01$ & $8.012 \mathrm{E}-05$ \\
1.2414 & $7.347 \mathrm{E}-05$ & $4.473 \mathrm{E}-02$ & $4.178 \mathrm{E}-01$ & $7.347 \mathrm{E}-05$ \\
1.4077 & $6.883 \mathrm{E}-05$ & $4.195 \mathrm{E}-02$ & $3.922 \mathrm{E}-01$ & $6.883 \mathrm{E}-05$ \\
1.6910 & $6.266 \mathrm{E}-05$ & $3.820 \mathrm{E}-02$ & $3.584 \mathrm{E}-01$ & $6.266 \mathrm{E}-05$ \\
1.8906 & $5.905 \mathrm{E}-05$ & $3.610 \mathrm{E}-02$ & $3.402 \mathrm{E}-01$ & $5.905 \mathrm{E}-05$ \\
2.1785 & $5.441 \mathrm{E}-05$ & $3.362 \mathrm{E}-02$ & $3.192 \mathrm{E}-01$ & $5.441 \mathrm{E}-05$ \\
2.2890 & $5.279 \mathrm{E}-05$ & $3.281 \mathrm{E}-02$ & $3.123 \mathrm{E}-01$ & $5.279 \mathrm{E}-05$ \\
2.4878 & $5.018 \mathrm{E}-05$ & $3.149 \mathrm{E}-02$ & $3.016 \mathrm{E}-01$ & $5.018 \mathrm{E}-05$ \\
2.6854 & $4.794 \mathrm{E}-05$ & $3.034 \mathrm{E}-02$ & $2.926 \mathrm{E}-01$ & $4.794 \mathrm{E}-05$ \\
2.9224 & $4.576 \mathrm{E}-05$ & $2.912 \mathrm{E}-02$ & $2.835 \mathrm{E}-01$ & $4.576 \mathrm{E}-05$ \\
3.2000 & $4.383 \mathrm{E}-05$ & $2.789 \mathrm{E}-02$ & $2.751 \mathrm{E}-01$ & $4.383 \mathrm{E}-05$ \\
\hline & & & &
\end{tabular}


HNF-SD-WM-CN-091
Rev. 0
Page 21 of 64

Organic Tank (H0-64-4278) Annular Source - Middle

Source Shields Distance to Detector, $X=7.800 \mathrm{E}+01 \mathrm{~cm}$

Annular $13 \quad$ Cyl. \& S1ab Source Volume $=4.963 \mathrm{E}+04 \mathrm{cc}$

Source Mass $=4.031 E+04$ grams

Source Length $=1.036 \mathrm{E}+03 \mathrm{~cm}$ Distance Along Cyl inder, $Y=5.180 \mathrm{E}+02 \mathrm{~cm}$

Integration Specs: $\quad$ NTHETA $=11$ NPSI $=11$ DELR computed internally

Total Intervals: $1.731 \mathrm{E}+02$ (photon source is the 2nd region)

Shield Thickness: $7.620 \mathrm{E}+01,1.000 \mathrm{E}-01,1.270 \mathrm{E}+00,4.300 \mathrm{E}-01 \mathrm{~cm}$

Distances from Dose Point to the Outside of

(1) Source Region: $1.700 \mathrm{E}+00 \mathrm{~cm}$ (2) Next Layer: $4.300 \mathrm{E}-01 \mathrm{~cm}$

Dose Buildup Data for Shield 3 with Effective Atomic Number 26.00

Buildup Material is Iron

Source values are interpreted as $\mu \mathrm{Ci}$

Source Scale Factor was $1.000 E+03$

Fluence-to-Dose Conversion: Photons in Air

\begin{tabular}{|c|c|c|c|c|}
\hline $\begin{array}{l}\text { Average } \\
\text { E, MeV }\end{array}$ & $\begin{array}{r}\text { Source Total } \\
\text { photons/sec }\end{array}$ & $\begin{array}{l}\text { Fluence to } \\
\text { Dose Factor }\end{array}$ & $\begin{array}{l}\text { Energy } \mathrm{Fl} \text { luence } \\
\mathrm{MeV} / \mathrm{cm}^{2} / \mathrm{s}\end{array}$ & $\begin{array}{c}\text { Dose Rate } \\
\mathrm{R} / \mathrm{hr}\end{array}$ \\
\hline $\begin{array}{l}0.0145 \\
0.0254 \\
0.0345 \\
0.0442 \\
0.0553 \\
0.0648 \\
0.0748 \\
0.0847 \\
0.0950 \\
0.1406 \\
0.2408 \\
0.3468 \\
0.5081 \\
0.6409 \\
0.8129 \\
1.0457 \\
1.2414 \\
1.4077 \\
1.6910 \\
1.8906 \\
2.1785 \\
2.2890 \\
2.4878 \\
2.6854 \\
2.9224 \\
3.2000\end{array}$ & $\begin{array}{l}1.490 \mathrm{E}+07 \\
9.971 \mathrm{E}+06 \\
9.163 \mathrm{E}+06 \\
4.916 \mathrm{E}+06 \\
3.038 \mathrm{E}+06 \\
2.273 \mathrm{E}+06 \\
1.785 \mathrm{E}+06 \\
2.904 \mathrm{E}+06 \\
1.370 \mathrm{E}+06 \\
1.353 \mathrm{E}+07 \\
3.749 \mathrm{E}+06 \\
1.906 \mathrm{E}+06 \\
5.572 \mathrm{E}+07 \\
5.733 \mathrm{E}+07 \\
3.255 \mathrm{E}+06 \\
4.703 \mathrm{E}+06 \\
1.199 \mathrm{E}+07 \\
2.088 \mathrm{E}+06 \\
5.269 \mathrm{E}+04 \\
3.066 \mathrm{E}+04 \\
2.201 \mathrm{E}+05 \\
9.159 \mathrm{E}+03 \\
4.520 \mathrm{E}+03 \\
1.996 \mathrm{E}+03 \\
9.697 \mathrm{E}+02 \\
7.528 \mathrm{E}+01\end{array}$ & $\begin{array}{l}9.036 \mathrm{E}-05 \\
1.577 \mathrm{E}-05 \\
6.335 \mathrm{E}-06 \\
3.359 \mathrm{E}-06 \\
2.204 \mathrm{E}-06 \\
1.835 \mathrm{E}-06 \\
1.666 \mathrm{E}-06 \\
1.605 \mathrm{E}-06 \\
1.592 \mathrm{E}-06 \\
1.708 \mathrm{E}-06 \\
1.919 \mathrm{E}-06 \\
2.017 \mathrm{E}-06 \\
2.040 \mathrm{E}-06 \\
2.030 \mathrm{E}-06 \\
1.986 \mathrm{E}-06 \\
1.907 \mathrm{E}-06 \\
1.845 \mathrm{E}-06 \\
1.794 \mathrm{E}-06 \\
1.714 \mathrm{E}-06 \\
1.662 \mathrm{E}-06 \\
1.604 \mathrm{E}-06 \\
1.593 \mathrm{E}-06 \\
1.537 \mathrm{E}-06 \\
1.501 \mathrm{E}-06 \\
1.464 \mathrm{E}-06 \\
1.425 \mathrm{E}-06\end{array}$ & $\begin{array}{l}0.000 \mathrm{E}+00 \\
0.000 \mathrm{E}+00 \\
2.671 \mathrm{E}-26 \\
4.401 \mathrm{E}-14 \\
1.735 \mathrm{E}-08 \\
3.203 \mathrm{E}-06 \\
7.680 \mathrm{E}-05 \\
1.021 \mathrm{E}-03 \\
2.058 \mathrm{E}-03 \\
6.858 \mathrm{E}-01 \\
8.498 \mathrm{E}-01 \\
8.303 \mathrm{E}-01 \\
4.343 \mathrm{E}+01 \\
6.210 \mathrm{E}+01 \\
4.864 \mathrm{E}+00 \\
9.712 \mathrm{E}+00 \\
3.055 \mathrm{E}+01 \\
6.171 \mathrm{E}+00 \\
1.917 \mathrm{E}-01 \\
1.262 \mathrm{E}-01 \\
1.060 \mathrm{E}+00 \\
4.657 \mathrm{E}-02 \\
2.511 \mathrm{E}-02 \\
1.198 \mathrm{E}-02 \\
6.324 \mathrm{E}-03 \\
5.366 \mathrm{E}-04\end{array}$ & $\begin{array}{l}0.000 \mathrm{E}+00 \\
0.000 \mathrm{E}+00 \\
1.692 \mathrm{E}-31 \\
1.478 \mathrm{E}-19 \\
3.822 \mathrm{E}-14 \\
5.878 \mathrm{E}-12 \\
1.280 \mathrm{E}-10 \\
1.638 \mathrm{E}-09 \\
3.277 \mathrm{E}-09 \\
1.172 \mathrm{E}-06 \\
1.631 \mathrm{E}-06 \\
1.675 \mathrm{E}-06 \\
8.859 \mathrm{E}-05 \\
1.260 \mathrm{E}-04 \\
9.660 \mathrm{E}-06 \\
1.852 \mathrm{E}-05 \\
5.635 \mathrm{E}-05 \\
1.107 \mathrm{E}-05 \\
3.287 \mathrm{E}-07 \\
2.098 \mathrm{E}-07 \\
1.701 \mathrm{E}-06 \\
7.420 \mathrm{E}-08 \\
3.861 \mathrm{E}-08 \\
1.799 \mathrm{E}-08 \\
9.261 \mathrm{E}-09 \\
7.646 \mathrm{E}-10\end{array}$ \\
\hline Totals & $2.049 \mathrm{E}+08$ & ons / sec & $1.607 E+02$ & $3.171 \mathrm{E}-04$ \\
\hline
\end{tabular}


HNF-SD-WM-CN-091

Rev. 0

Page 22 of 64

Organic Tank (HO-64-4278) Line Source - End

Shield Composition, g/cc

Shield 1 Shield 2 Shield 3

\begin{tabular}{lccc}
\hline AIR & $0.000 \mathrm{E}+00$ & $0.000 \mathrm{E}+00$ & $1.290 \mathrm{E}-03$ \\
ALUMINUM & $8.122 \mathrm{E}-01$ & $0.000 \mathrm{E}+00$ & $0.000 \mathrm{E}+00$ \\
IRON & $0.000 \mathrm{E}+00$ & $7.800 \mathrm{E}+00$ & $0.000 \mathrm{E}+00$ \\
\hline Totals: & $8.122 \mathrm{E}-01$ & $7.800 \mathrm{E}+00$ & $1.290 \mathrm{E}-03$ \\
\hline E, MeV & Linear Attenuation, per cm (1ast \\
\hline 0.0145 & $7.175 \mathrm{E}+00$ & $5.015 \mathrm{E}+02$ & $2.205 \mathrm{E}-03$ \\
0.0254 & $1.430 \mathrm{E}+00$ & $1.038 \mathrm{E}+02$ & $5.985 \mathrm{E}-04$ \\
0.0345 & $6.386 \mathrm{E}-01$ & $4.319 \mathrm{E}+01$ & $3.741 \mathrm{E}-04$ \\
0.0442 & $3.703 \mathrm{E}-01$ & $2.131 \mathrm{E}+01$ & $2.891 \mathrm{E}-04$ \\
0.0553 & $2.476 \mathrm{E}-01$ & $1.155 \mathrm{E}+01$ & $2.486 \mathrm{E}-04$ \\
0.0648 & $1.992 \mathrm{E}-01$ & $7.729 \mathrm{E}+00$ & $2.318 \mathrm{E}-04$ \\
0.0748 & $1.714 \mathrm{E}-01$ & $5.439 \mathrm{E}+00$ & $2.186 \mathrm{E}-04$ \\
0.0847 & $1.543 \mathrm{E}-01$ & $4.095 \mathrm{E}+00$ & $2.092 \mathrm{E}-04$ \\
0.0950 & $1.419 \mathrm{E}-01$ & $3.215 \mathrm{E}+00$ & $2.019 \mathrm{E}-04$ \\
0.1406 & $1.159 \mathrm{E}-01$ & $1.698 \mathrm{E}+00$ & $1.793 \mathrm{E}-04$ \\
0.2408 & $9.207 \mathrm{E}-02$ & $9.921 \mathrm{E}-01$ & $1.496 \mathrm{E}-04$ \\
0.3468 & $7.972 \mathrm{E}-02$ & $7.904 \mathrm{E}-01$ & $1.302 \mathrm{E}-04$ \\
0.5081 & $6.816 \mathrm{E}-02$ & $6.501 \mathrm{E}-01$ & $1.113 \mathrm{E}-04$ \\
0.6409 & $6.148 \mathrm{E}-02$ & $5.808 \mathrm{E}-01$ & $1.007 \mathrm{E}-04$ \\
0.8129 & $5.512 \mathrm{E}-02$ & $5.170 \mathrm{E}-01$ & $9.044 \mathrm{E}-05$ \\
1.0457 & $4.876 \mathrm{E}-02$ & $4.559 \mathrm{E}-01$ & $8.012 \mathrm{E}-05$ \\
1.2414 & $4.473 \mathrm{E}-02$ & $4.178 \mathrm{E}-01$ & $7.347 \mathrm{E}-05$ \\
1.4077 & $4.195 \mathrm{E}-02$ & $3.922 \mathrm{E}-01$ & $6.883 \mathrm{E}-05$ \\
1.6910 & $3.820 \mathrm{E}-02$ & $3.584 \mathrm{E}-01$ & $6.266 \mathrm{E}-05$ \\
1.8906 & $3.610 \mathrm{E}-02$ & $3.402 \mathrm{E}-01$ & $5.905 \mathrm{E}-05$ \\
2.1785 & $3.362 \mathrm{E}-02$ & $3.192 \mathrm{E}-01$ & $5.441 \mathrm{E}-05$ \\
2.2890 & $3.281 \mathrm{E}-02$ & $3.123 \mathrm{E}-01$ & $5.279 \mathrm{E}-05$ \\
2.4878 & $3.149 \mathrm{E}-02$ & $3.016 \mathrm{E}-01$ & $5.018 \mathrm{E}-05$ \\
2.6854 & $3.034 \mathrm{E}-02$ & $2.926 \mathrm{E}-01$ & $4.794 \mathrm{E}-05$ \\
2.9224 & $2.912 \mathrm{E}-02$ & $2.835 \mathrm{E}-01$ & $4.576 \mathrm{E}-05$ \\
3.2000 & $2.789 \mathrm{E}-02$ & $2.751 \mathrm{E}-01$ & $4.383 \mathrm{E}-05$ \\
\hline & & & \\
\hline & & &
\end{tabular}




$$
\begin{array}{r}
\text { HNF-SD-WM-CN-091 } \\
\text { Rev. } 0 \\
\text { Page 23 of } 64
\end{array}
$$

Organic Tank (H0-64-4278) Line Source - End

Source Shields Distance to Detector, $X=2.500 \mathrm{E}+00 \mathrm{~cm}$

Cylindrical Cylindrical Source Volume $=3.255 \mathrm{E}+03 \mathrm{cc}$

Source Mass $=2.643 \mathrm{E}+03$ grams

Source Length $=1.036 \mathrm{E}+03 \mathrm{~cm} \quad$ Distance Along $\mathrm{Cyl}$ inder, $\mathrm{Y}=1.035 \mathrm{E}+03 \mathrm{~cm}$

Iritegration Specs: $\quad$ NTHETA $=11$ NPSI $=11$ DELR computed internally

Total Intervals: $7.623 E+04$ (photon source is the lst region)

Shield Thickness: $1.000 E+00,1.270 E+00,2.300 E-01 \mathrm{~cm}$

Distances from Dose Point to the Outside of

$\begin{array}{ll}\text { (1) Source Region: } 1.500 \mathrm{E}+00 \mathrm{~cm} & \text { (2) Next Layer: } 2.300 \mathrm{E}-01 \mathrm{~cm}\end{array}$

Dose Buildup Data for Shield 2 with Effective Atomic Number 26.00

Buildup Material is Iron

Source values are interpreted as $\mu \mathrm{C} i$

Source Scale Factor was 1.000E+03

Fluence-to-Dose Conversion: Photons in Air

\begin{tabular}{ccccc}
$\begin{array}{c}\text { Average } \\
\text { E, MeV }\end{array}$ & $\begin{array}{c}\text { Source Total } \\
\text { photons } / \mathrm{sec}\end{array}$ & $\begin{array}{c}\text { Fluence to } \\
\text { Dose Factor }\end{array}$ & $\begin{array}{c}\text { Energy Fluence } \\
\text { MeV } / \mathrm{cm} 2 / \mathrm{s}\end{array}$ & $\begin{array}{c}\text { Dose Rate } \\
\text { R/hr }\end{array}$ \\
\hline 0.0145 & $1.490 \mathrm{E}+07$ & $9.036 \mathrm{E}-05$ & $0.000 \mathrm{E}+00$ & $0.000 \mathrm{E}+00$ \\
0.0254 & $9.971 \mathrm{E}+06$ & $1.577 \mathrm{E}-05$ & $0.000 \mathrm{E}+00$ & $0.000 \mathrm{E}+00$ \\
0.0345 & $9.163 \mathrm{E}+06$ & $6.335 \mathrm{E}-06$ & $1.495 \mathrm{E}-24$ & $9.472 \mathrm{E}-30$ \\
0.0442 & $4.916 \mathrm{E}+06$ & $3.359 \mathrm{E}-06$ & $2.830 \mathrm{E}-12$ & $9.507 \mathrm{E}-18$ \\
0.0553 & $3.038 \mathrm{E}+06$ & $2.204 \mathrm{E}-06$ & $1.119 \mathrm{E}-06$ & $2.467 \mathrm{E}-12$ \\
0.0648 & $2.273 \mathrm{E}+06$ & $1.835 \mathrm{E}-06$ & $1.962 \mathrm{E}-04$ & $3.601 \mathrm{E}-10$ \\
0.0748 & $1.785 \mathrm{E}+06$ & $1.666 \mathrm{E}-06$ & $4.351 \mathrm{E}-03$ & $7.251 \mathrm{E}-09$ \\
0.0847 & $2.904 \mathrm{E}+06$ & $1.605 \mathrm{E}-06$ & $5.331 \mathrm{E}-02$ & $8.553 \mathrm{E}-08$ \\
0.0950 & $1.370 \mathrm{E}+06$ & $1.592 \mathrm{E}-06$ & $9.925 \mathrm{E}-02$ & $1.580 \mathrm{E}-07$ \\
0.1406 & $1.353 \mathrm{E}+07$ & $1.708 \mathrm{E}-06$ & $2.451 \mathrm{E}+01$ & $4.187 \mathrm{E}-05$ \\
0.2408 & $3.749 \mathrm{E}+06$ & $1.919 \mathrm{E}-06$ & $2.406 \mathrm{E}+01$ & $4.616 \mathrm{E}-05$ \\
0.3468 & $1.906 \mathrm{E}+06$ & $2.017 \mathrm{E}-06$ & $2.140 \mathrm{E}+01$ & $4.316 \mathrm{E}-05$ \\
0.5081 & $5.572 \mathrm{E}+07$ & $2.040 \mathrm{E}-06$ & $1.039 \mathrm{E}+03$ & $2.119 \mathrm{E}-03$ \\
0.6409 & $5.733 \mathrm{E}+07$ & $2.030 \mathrm{E}-06$ & $1.427 \mathrm{E}+03$ & $2.896 \mathrm{E}-03$ \\
0.8129 & $3.255 \mathrm{E}+06$ & $1.986 \mathrm{E}-06$ & $1.076 \mathrm{E}+02$ & $2.136 \mathrm{E}-04$ \\
1.0457 & $4.703 \mathrm{E}+06$ & $1.907 \mathrm{E}-06$ & $2.069 \mathrm{E}+02$ & $3.944 \mathrm{E}-04$ \\
1.2414 & $1.199 \mathrm{E}+07$ & $1.845 \mathrm{E}-06$ & $6.359 \mathrm{E}+02$ & $1.173 \mathrm{E}-03$ \\
1.4077 & $2.088 \mathrm{E}+06$ & $1.794 \mathrm{E}-06$ & $1.265 \mathrm{E}+02$ & $2.269 \mathrm{E}-04$ \\
1.6910 & $5.269 \mathrm{E}+04$ & $1.714 \mathrm{E}-06$ & $3.855 \mathrm{E}+00$ & $6.609 \mathrm{E}-06$ \\
1.8906 & $3.066 \mathrm{E}+04$ & $1.662 \mathrm{E}-06$ & $2.512 \mathrm{E}+00$ & $4.176 \mathrm{E}-06$ \\
2.1785 & $2.201 \mathrm{E}+05$ & $1.604 \mathrm{E}-06$ & $2.085 \mathrm{E}+01$ & $3.345 \mathrm{E}-05$ \\
2.2890 & $9.159 \mathrm{E}+03$ & $1.593 \mathrm{E}-06$ & $9.126 \mathrm{E}-01$ & $1.454 \mathrm{E}-06$ \\
2.4878 & $4.520 \mathrm{E}+03$ & $1.537 \mathrm{E}-06$ & $4.894 \mathrm{E}-01$ & $7.523 \mathrm{E}-07$ \\
2.6854 & $1.996 \mathrm{E}+03$ & $1.501 \mathrm{E}-06$ & $2.326 \mathrm{E}-01$ & $3.492 \mathrm{E}-07$ \\
2.9224 & $9.697 \mathrm{E}+02$ & $1.464 \mathrm{E}-06$ & $1.224 \mathrm{E}-01$ & $1.792 \mathrm{E}-07$ \\
3.2000 & $7.528 \mathrm{E}+01$ & $1.425 \mathrm{E}-06$ & $1.036 \mathrm{E}-02$ & $1.476 \mathrm{E}-08$ \\
\hline Tota15: & $2.049 \mathrm{E}+08$ & $\mathrm{phot} 0 \mathrm{5} / \mathrm{sec}$ & $3.641 \mathrm{E}+03$ & $7.201 \mathrm{E}-03 \mathrm{R} / \mathrm{hr}$
\end{tabular}


HNF-SD-WM-CN-091

Rev. 0

Page 24 of 64

Organic Tank (HO-64-4278) Annular Source - End

Shield Composition, g/cc

Shield 1 Shield 2 Shield 3 Shield 4

\begin{tabular}{lcccc}
\hline AIR & $1.290 \mathrm{E}-03$ & $0.000 \mathrm{E}+00$ & $0.000 \mathrm{E}+00$ & $1.290 \mathrm{E}-03$ \\
ALUMINUM & $0.000 \mathrm{E}+00$ & $8.122 \mathrm{E}-01$ & $0.000 \mathrm{E}+00$ & $0.000 \mathrm{E}+00$ \\
IRON & $0.000 \mathrm{E}+00$ & $0.000 \mathrm{E}+00$ & $7.800 \mathrm{E}+00$ & $0.000 \mathrm{E}+00$ \\
\hline Totals: & $1.290 \mathrm{E}-03$ & $8.122 \mathrm{E}-01$ & $7.800 \mathrm{E}+00$ & $1.290 \mathrm{E}-03$ \\
\hline E, MeV & Linear Attenuation, per cm (7ast & region is a \\
\hline 0.0145 & $2.205 \mathrm{E}-03$ & $7.175 \mathrm{E}+00$ & $5.015 \mathrm{E}+02$ & $2.205 \mathrm{E}-03$ \\
0.0254 & $5.985 \mathrm{E}-04$ & $1.430 \mathrm{E}+00$ & $1.038 \mathrm{E}+02$ & $5.985 \mathrm{E}-04$ \\
0.0345 & $3.741 \mathrm{E}-04$ & $6.386 \mathrm{E}-01$ & $4.319 \mathrm{E}+01$ & $3.741 \mathrm{E}-04$ \\
0.0442 & $2.891 \mathrm{E}-04$ & $3.703 \mathrm{E}-01$ & $2.131 \mathrm{E}+01$ & $2.891 \mathrm{E}-04$ \\
0.0553 & $2.486 \mathrm{E}-04$ & $2.476 \mathrm{E}-01$ & $1.155 \mathrm{E}+01$ & $2.486 \mathrm{E}-04$ \\
0.0648 & $2.318 \mathrm{E}-04$ & $1.992 \mathrm{E}-01$ & $7.729 \mathrm{E}+00$ & $2.318 \mathrm{E}-04$ \\
0.0748 & $2.186 \mathrm{E}-04$ & $1.714 \mathrm{E}-01$ & $5.439 \mathrm{E}+00$ & $2.186 \mathrm{E}-04$ \\
0.0847 & $2.092 \mathrm{E}-04$ & $1.543 \mathrm{E}-01$ & $4.095 \mathrm{E}+00$ & $2.092 \mathrm{E}-04$ \\
0.0950 & $2.019 \mathrm{E}-04$ & $1.419 \mathrm{E}-01$ & $3.215 \mathrm{E}+00$ & $2.019 \mathrm{E}-04$ \\
0.1406 & $1.793 \mathrm{E}-04$ & $1.159 \mathrm{E}-01$ & $1.698 \mathrm{E}+00$ & $1.793 \mathrm{E}-04$ \\
0.2408 & $1.496 \mathrm{E}-04$ & $9.207 \mathrm{E}-02$ & $9.921 \mathrm{E}-01$ & $1.496 \mathrm{E}-04$ \\
0.3468 & $1.302 \mathrm{E}-04$ & $7.972 \mathrm{E}-02$ & $7.904 \mathrm{E}-01$ & $1.302 \mathrm{E}-04$ \\
0.5081 & $1.113 \mathrm{E}-04$ & $6.816 \mathrm{E}-02$ & $6.501 \mathrm{E}-01$ & $1.113 \mathrm{E}-04$ \\
0.6409 & $1.007 \mathrm{E}-04$ & $6.148 \mathrm{E}-02$ & $5.808 \mathrm{E}-01$ & $1.007 \mathrm{E}-04$ \\
0.8129 & $9.044 \mathrm{E}-05$ & $5.512 \mathrm{E}-02$ & $5.170 \mathrm{E}-01$ & $9.044 \mathrm{E}-05$ \\
1.0457 & $8.012 \mathrm{E}-05$ & $4.876 \mathrm{E}-02$ & $4.559 \mathrm{E}-01$ & $8.012 \mathrm{E}-05$ \\
1.2414 & $7.347 \mathrm{E}-05$ & $4.473 \mathrm{E}-02$ & $4.178 \mathrm{E}-01$ & $7.347 \mathrm{E}-05$ \\
1.4077 & $6.883 \mathrm{E}-05$ & $4.195 \mathrm{E}-02$ & $3.922 \mathrm{E}-01$ & $6.883 \mathrm{E}-05$ \\
1.6910 & $6.266 \mathrm{E}-05$ & $3.820 \mathrm{E}-02$ & $3.584 \mathrm{E}-01$ & $6.266 \mathrm{E}-05$ \\
1.8906 & $5.905 \mathrm{E}-05$ & $3.610 \mathrm{E}-02$ & $3.402 \mathrm{E}-01$ & $5.905 \mathrm{E}-05$ \\
2.1785 & $5.441 \mathrm{E}-05$ & $3.362 \mathrm{E}-02$ & $3.192 \mathrm{E}-01$ & $5.441 \mathrm{E}-05$ \\
2.2890 & $5.279 \mathrm{E}-05$ & $3.281 \mathrm{E}-02$ & $3.123 \mathrm{E}-01$ & $5.279 \mathrm{E}-05$ \\
2.4878 & $5.018 \mathrm{E}-05$ & $3.149 \mathrm{E}-02$ & $3.016 \mathrm{E}-01$ & $5.018 \mathrm{E}-05$ \\
2.6854 & $4.794 \mathrm{E}-05$ & $3.034 \mathrm{E}-02$ & $2.926 \mathrm{E}-01$ & $4.794 \mathrm{E}-05$ \\
2.9224 & $4.576 \mathrm{E}-05$ & $2.912 \mathrm{E}-02$ & $2.835 \mathrm{E}-01$ & $4.576 \mathrm{E}-05$ \\
3.2000 & $4.383 \mathrm{E}-05$ & $2.789 \mathrm{E}-02$ & $2.751 \mathrm{E}-01$ & $4.383 \mathrm{E}-05$ \\
\hline & & & &
\end{tabular}


HNF-SD-WM-CN-091

Rev. 0

Page 25 of 64

Organic Tank (H0-64-4278) Annular Source - End

Source Shields Distance to Detector, $X=7.800 \mathrm{E}+01 \mathrm{~cm}$

Annular $13 \quad$ Cyl. \& Slab Source Volume $=4.963 \mathrm{E}+04 \mathrm{cc}$

Source Mass $=4.031 \mathrm{E}+04$ grams

Source Length $=1.036 E+03 \mathrm{~cm} \quad$ Distance Along Cylinder, $Y=1.035 E+03 \mathrm{~cm}$

Integration Specs: $\quad$ NTHETA $=11 \quad$ NPSI $=11$ DELR computed internaliy

Total Intervals: $1.731 E+02$ (photon source is the 2 nd region)

Shield Thickness: $7.620 \mathrm{E}+01,1.000 \mathrm{E}-01,1.270 \mathrm{E}+00,4.300 \mathrm{E}-01 \mathrm{~cm}$

Distances from Dose Point to the Outside of

(1) Source Region: $1.700 \mathrm{E}+00 \mathrm{~cm}$ (2) Next Layer: $4.300 \mathrm{E}-01 \mathrm{~cm}$

Dose Buildup Data for Shield 3 with Effective Atomic Number 26.00

Buildup Material is Iron

Source values are interpreted as $\mu \mathrm{Ci}$

Source Scale Factor was $1.000 \mathrm{E}+03$

Fluence-to-Dose Conversion: Photons in Air

\begin{tabular}{ccccc}
$\begin{array}{r}\text { Average } \\
\text { E, MeV }\end{array}$ & $\begin{array}{c}\text { Source Total } \\
\text { photons } / \mathrm{sec}\end{array}$ & $\begin{array}{c}\text { Fluence to } \\
\text { Dose Factor }\end{array}$ & $\begin{array}{c}\text { Energy Fluence } \\
\text { MeV } / \mathrm{cm}^{2} / \mathrm{s}\end{array}$ & $\begin{array}{c}\text { Dose Rate } \\
\text { R/hr }\end{array}$ \\
\hline 0.0145 & $1.490 \mathrm{E}+07$ & $9.036 \mathrm{E}-05$ & $0.000 \mathrm{E}+00$ & $0.000 \mathrm{E}+00$ \\
0.0254 & $9.971 \mathrm{E}+06$ & $1.577 \mathrm{E}-05$ & $0.000 \mathrm{E}+00$ & $0.000 \mathrm{E}+00$ \\
0.0345 & $9.163 \mathrm{E}+06$ & $6.335 \mathrm{E}-06$ & $2.263 \mathrm{E}-26$ & $1.433 \mathrm{E}-31$ \\
0.0442 & $4.916 \mathrm{E}+06$ & $3.359 \mathrm{E}-06$ & $3.621 \mathrm{E}-14$ & $1.216 \mathrm{E}-19$ \\
0.0553 & $3.038 \mathrm{E}+06$ & $2.204 \mathrm{E}-06$ & $1.396 \mathrm{E}-08$ & $3.075 \mathrm{E}-14$ \\
0.0648 & $2.273 \mathrm{E}+06$ & $1.835 \mathrm{E}-06$ & $2.526 \mathrm{E}-06$ & $4.636 \mathrm{E}-12$ \\
0.0748 & $1.785 \mathrm{E}+06$ & $1.666 \mathrm{E}-06$ & $5.914 \mathrm{E}-05$ & $9.855 \mathrm{E}-11$ \\
0.0847 & $2.904 \mathrm{E}+06$ & $1.605 \mathrm{E}-06$ & $7.680 \mathrm{E}-04$ & $1.232 \mathrm{E}-09$ \\
0.0950 & $1.370 \mathrm{E}+06$ & $1.592 \mathrm{E}-06$ & $1.516 \mathrm{E}-03$ & $2.413 \mathrm{E}-09$ \\
0.1406 & $1.353 \mathrm{E}+07$ & $1.708 \mathrm{E}-06$ & $4.702 \mathrm{E}-01$ & $8.031 \mathrm{E}-07$ \\
0.2408 & $3.749 \mathrm{E}+06$ & $1.919 \mathrm{E}-06$ & $5.583 \mathrm{E}-01$ & $1.071 \mathrm{E}-06$ \\
0.3468 & $1.906 \mathrm{E}+06$ & $2.017 \mathrm{E}-06$ & $5.376 \mathrm{E}-01$ & $1.084 \mathrm{E}-06$ \\
0.5081 & $5.572 \mathrm{E}+07$ & $2.040 \mathrm{E}-06$ & $2.783 \mathrm{E}+01$ & $5.676 \mathrm{E}-05$ \\
0.6409 & $5.733 \mathrm{E}+07$ & $2.030 \mathrm{E}-06$ & $3.959 \mathrm{E}+01$ & $8.035 \mathrm{E}-05$ \\
0.8129 & $3.255 \mathrm{E}+06$ & $1.986 \mathrm{E}-06$ & $3.086 \mathrm{E}+00$ & $6.129 \mathrm{E}-06$ \\
1.0457 & $4.703 \mathrm{E}+06$ & $1.907 \mathrm{E}-06$ & $6.136 \mathrm{E}+00$ & $1.170 \mathrm{E}-05$ \\
1.2414 & $1.199 \mathrm{E}+07$ & $1.845 \mathrm{E}-06$ & $1.925 \mathrm{E}+01$ & $3.551 \mathrm{E}-05$ \\
1.4077 & $2.088 \mathrm{E}+06$ & $1.794 \mathrm{E}-06$ & $3.883 \mathrm{E}+00$ & $6.965 \mathrm{E}-06$ \\
1.6910 & $5.269 \mathrm{E}+04$ & $1.714 \mathrm{E}-06$ & $1.204 \mathrm{E}-01$ & $2.064 \mathrm{E}-07$ \\
1.8906 & $3.066 \mathrm{E}+04$ & $1.662 \mathrm{E}-06$ & $7.917 \mathrm{E}-02$ & $1.316 \mathrm{E}-07$ \\
2.1785 & $2.201 \mathrm{E}+05$ & $1.604 \mathrm{E}-06$ & $6.642 \mathrm{E}-01$ & $1.066 \mathrm{E}-06$ \\
2.2890 & $9.159 \mathrm{E}+03$ & $1.593 \mathrm{E}-06$ & $2.917 \mathrm{E}-02$ & $4.648 \mathrm{E}-08$ \\
2.4878 & $4.520 \mathrm{E}+03$ & $1.537 \mathrm{E}-06$ & $1.572 \mathrm{E}-02$ & $2.417 \mathrm{E}-08$ \\
2.6854 & $1.996 \mathrm{E}+03$ & $1.501 \mathrm{E}-06$ & $7.499 \mathrm{E}-03$ & $1.126 \mathrm{E}-08$ \\
2.9224 & $9.697 \mathrm{E}+02$ & $1.464 \mathrm{E}-06$ & $3.957 \mathrm{E}-03$ & $5.794 \mathrm{E}-09$ \\
3.2000 & $7.528 \mathrm{E}+01$ & $1.425 \mathrm{E}-06$ & $3.356 \mathrm{E}-04$ & $4.782 \mathrm{E}-10$ \\
\hline
\end{tabular}

Totals: $2.049 \mathrm{E}+08$ photons $/ \mathrm{sec} \quad 1.023 \mathrm{E}+02 \quad 2.019 \mathrm{E}-04 \mathrm{R} / \mathrm{hr}$ 
HNF-SD-WM-CN-091

Rev. 0

Page 26 of 64

0ld UNH Tank (H0-64-5920) Line Source - Middle

Table of Source Activity:

Scale Factor $=1.000 E+03$

\begin{tabular}{|c|c|c|c|}
\hline \multicolumn{2}{|c|}{$\begin{array}{l}\text { I sotope } \\
\text { Wt. Name }\end{array}$} & $\begin{array}{l}\text { Initial } \\
\text { Varues }\end{array}$ & $\begin{array}{l}\text { Final } \\
\mu \mathrm{Ci}\end{array}$ \\
\hline 117 & $Z R-95$ & $7.180 \mathrm{E}-02$ & $7.180 E+01$ \\
\hline 119 & NB- 95 & $4.670 \mathrm{E}-01$ & $4.670 \mathrm{E}+02$ \\
\hline 155 & RU-103 & $3.960 \mathrm{E}-02$ & $3.960 \mathrm{E}+01$ \\
\hline 170 & RU-106 & $5.330 E-01$ & $5.330 E+02$ \\
\hline 172 & RH- 106 & $5.330 E-01$ & $5.330 \mathrm{E}+02$ \\
\hline 319 & CS-134 & $4.270 E-02$ & $4.270 E+01$ \\
\hline 335 & $\mathrm{CS}-137$ & $3.600 \mathrm{E}-02$ & $3.600 E+01$ \\
\hline 336 & $\mathrm{BA}-137 \mathrm{M}$ & $3.410 \mathrm{E}-02$ & $3.410 E+01$ \\
\hline 376 & $C E-144$ & $5.810 E-01$ & $5.810 E+02$ \\
\hline 377 & PR-144 & $5.810 \mathrm{E}-01$ & $5.810 \mathrm{E}+02$ \\
\hline 520 & $U-234$ & $2.690 \mathrm{E}-03$ & $2.690 \mathrm{E}+00$ \\
\hline 476 & $U-235$ & $9.800 E-02$ & $9.800 \mathrm{E}+01$ \\
\hline 398 & $U-236$ & 2. $170 \mathrm{E}-02$ & $2.170 \mathrm{E}+01$ \\
\hline 526 & $U-238$ & $1.590 \mathrm{E}+01$ & $1.590 \mathrm{E}+04$ \\
\hline
\end{tabular}


HNF-SD-WM-CN-091

Rev. 0

Page 27 of 64

01d UNH Tank (H0-64-5920) Line Source - Middle

Photon Production Rate for Each Radionuclide:

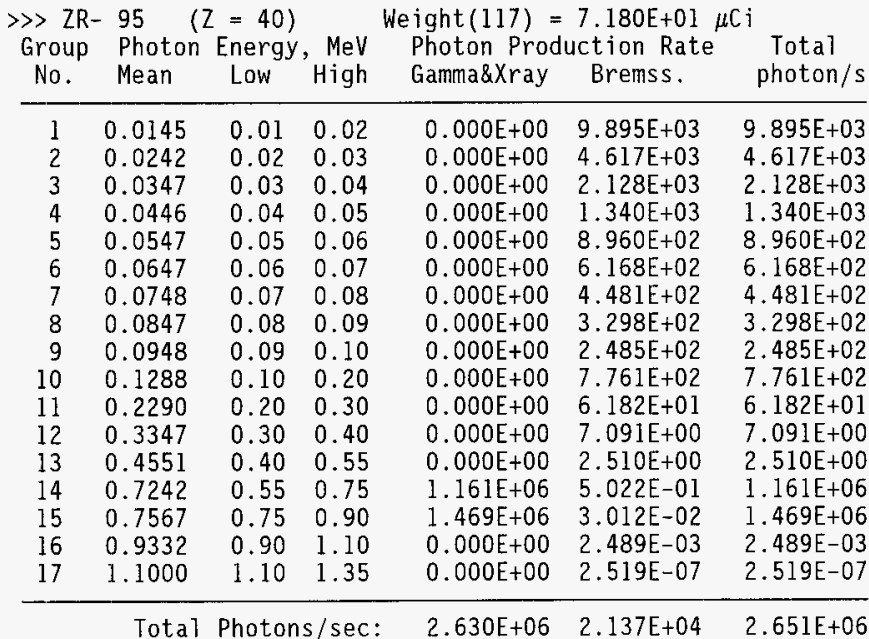

$\gg$ NB- $95 \quad(Z=41) \quad$ Weight $(119)=4.670 \mathrm{E}+02 \mu \mathrm{Ci}$

Group Photon Energy, MeV Photon Production Rate Total

No. Mean Low High Gamma\&Xray Bremss. photon/s

\begin{tabular}{rllllll}
\hline 1 & 0.0136 & 0.01 & 0.02 & $0.000 \mathrm{E}+00$ & $1.548 \mathrm{E}+04$ & $1.548 \mathrm{E}+04$ \\
2 & 0.0244 & 0.02 & 0.03 & $0.000 \mathrm{E}+00$ & $4.982 \mathrm{E}+03$ & $4.982 \mathrm{E}+03$ \\
3 & 0.0344 & 0.03 & 0.04 & $0.000 \mathrm{E}+00$ & $2.280 \mathrm{E}+03$ & $2.280 \mathrm{E}+03$ \\
4 & 0.0444 & 0.04 & 0.05 & $0.000 \mathrm{E}+00$ & $1.155 \mathrm{E}+03$ & $1.155 \mathrm{E}+03$ \\
5 & 0.0545 & 0.05 & 0.06 & $0.000 \mathrm{E}+00$ & $6.127 \mathrm{E}+02$ & $6.127 \mathrm{E}+02$ \\
6 & 0.0645 & 0.06 & 0.07 & $0.000 \mathrm{E}+00$ & $3.260 \mathrm{E}+02$ & $3.260 \mathrm{E}+02$ \\
7 & 0.0745 & 0.07 & 0.08 & $0.000 \mathrm{E}+00$ & $1.712 \mathrm{E}+02$ & $1.712 \mathrm{E}+02$ \\
8 & 0.0844 & 0.08 & 0.09 & $0.000 \mathrm{E}+00$ & $8.793 \mathrm{E}+01$ & $8.793 \mathrm{E}+01$ \\
9 & 0.0944 & 0.09 & 0.10 & $0.000 \mathrm{E}+00$ & $4.334 \mathrm{E}+01$ & $4.334 \mathrm{E}+01$ \\
10 & 0.1003 & 0.10 & 0.20 & $0.000 \mathrm{E}+00$ & $7.036 \mathrm{E}+01$ & $7.036 \mathrm{E}+01$ \\
1.1 & 0.2144 & 0.20 & 0.30 & $0.000 \mathrm{E}+00$ & $2.096 \mathrm{E}-02$ & $2.096 \mathrm{E}-02$ \\
12 & 0.3000 & 0.30 & 0.40 & $0.000 \mathrm{E}+00$ & $1.220 \mathrm{E}-05$ & $1.220 \mathrm{E}-05$ \\
1.5 & 0.7658 & 0.75 & 0.90 & $1.725 \mathrm{E}+07$ & $0.000 \mathrm{E}+00$ & $1.725 \mathrm{E}+07$ \\
\hline
\end{tabular}

Total Photons/sec: $1.725 \mathrm{E}+07 \quad 2.521 \mathrm{E}+04 \quad 1.727 \mathrm{E}+07$ 
HNF-SD-WM-CN-091

Rev. 0

Page 28 of 64

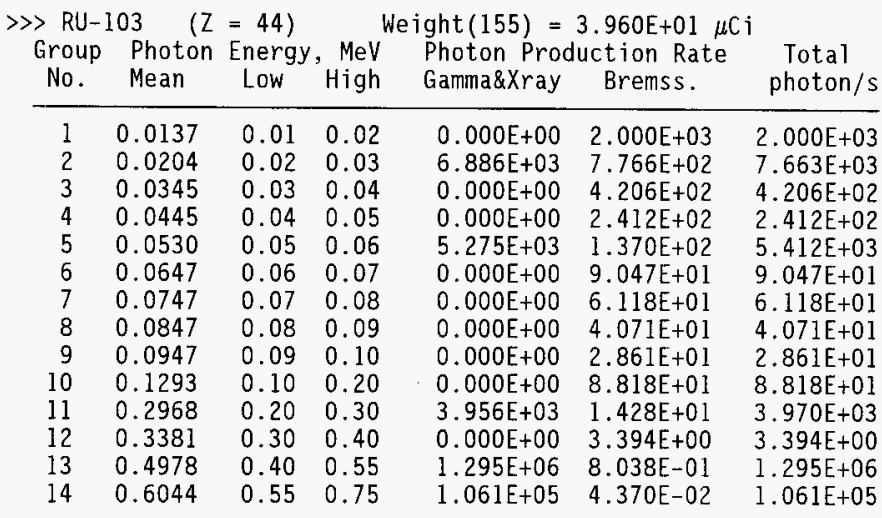

Total Photons/sec: $\quad 1.417 \mathrm{E}+06 \quad 3.903 \mathrm{E}+03 \quad 1.421 \mathrm{E}+06$

\begin{tabular}{|c|c|c|c|c|c|c|}
\hline $\begin{array}{l}\gg \mathrm{RU} \\
\text { Group } \\
\text { No. }\end{array}$ & $\begin{array}{l}\text { Photon } \\
\text { Mean }\end{array}$ & $\begin{array}{c}=44) \\
\text { Energy } \\
\text { Low }\end{array}$ & $\begin{array}{l}\text {, MeV } \\
\mathrm{High}\end{array}$ & $\begin{array}{l}\text { eight }(170)= \\
\text { Photon Prod } \\
\text { Gamma\&Xray }\end{array}$ & $\begin{array}{c}5.330 \mathrm{E}+02 \\
\text { uction Rate } \\
\text { Bremss. }\end{array}$ & $\begin{array}{c}\text { Total } \\
\text { photon/s }\end{array}$ \\
\hline $\begin{array}{l}1 \\
2 \\
3\end{array}$ & 0.0 & $\begin{array}{l}0.02 \\
0.03\end{array}$ & $\begin{array}{l}0.03 \\
0.04\end{array}$ & & 4. & \\
\hline
\end{tabular}

Total Photons/sec: $\quad 0.000 E+00 \quad 7.230 E+02 \quad 7.230 E+02$ 
HNF-SD-WM-CN-09]

Rev. 0

Page 29 of 64

\begin{tabular}{|c|c|c|c|c|c|c|}
\hline $\begin{array}{l}\text { GH- } \\
\text { Group } \\
\text { No. }\end{array}$ & $\begin{array}{l}96 \quad \text { (Z } \\
\text { Photon } \\
\text { Mean }\end{array}$ & $\begin{array}{l}=45) \\
\text { Energy } \\
\text { Low }\end{array}$ & $\begin{array}{l}\mathrm{MeV} \\
\mathrm{High}\end{array}$ & $\begin{array}{l}\text { ght }(172)= \\
\text { Photon Proo } \\
\text { Gamma\&Xray }\end{array}$ & $\begin{array}{c}.330 E+02 \mu \\
\text { ction Rate } \\
\text { Bremss. }\end{array}$ & $\begin{array}{l}\text { Total } \\
\text { photon/s }\end{array}$ \\
\hline 1 & 0 & & 0.02 & +00 & $1.020 E+06$ & .020 \\
\hline 2 & & & 0.03 & & J.7ust & \\
\hline 3 & 347 & 0.03 & 0.04 & $00 E+00$ & $3.616 \mathrm{E}+$ & $E+05$ \\
\hline 4 & 0. & 0.04 & 0.05 & $00 E+00$ & $76 \mathrm{E}+$ & +05 \\
\hline 5 & 0. & 0.05 & 0.06 & $E+00$ & $1.952 E+05$ & +05 \\
\hline 6 & & 0.06 & 0.07 & +00 & 1. & \\
\hline 7 & 0.0748 & 0.07 & 0.08 & $E+00$ & 1. $276 \mathrm{E}+$ & +05 \\
\hline 8 & 0. & 0.08 & 0.09 & $E+00$ & $74 \mathrm{E}$ & \\
\hline 9 & 0. & 0.09 & 0.10 & $E+00$ & $09 \mathrm{E}+$ & \\
\hline 10 & & 0.10 & 0.20 & $E+00$ & $6.946 \mathrm{E}+$ & \\
\hline 11 & 0. & 0.20 & 0.30 & $E+00$ & $98 \mathrm{E}+$ & \\
\hline 12 & 0.3 & 0.30 & 0.40 & $E+00$ & 1 . & \\
\hline 13 & & 0.40 & 0.55 & $E+06$ & $4 E+05$ & \\
\hline 14 & & 0.55 & 0.75 & $71 \mathrm{E}+06$ & $7.271 \mathrm{E}+$ & 2. \\
\hline 15 & 0.8 & 0.75 & 0.90 & $3 E+04$ & $3.052 \mathrm{E}+04$ & 1 . \\
\hline 16 & & 0.90 & 1.10 & $2 E+05$ & $2.467 \mathrm{E}+$ & \\
\hline 17 & 1.1428 & 1.10 & 1.35 & $7.888 \mathrm{E}+04$ & $1.708 \mathrm{E}+$ & 9. \\
\hline 18 & 1.4048 & 1.35 & 1.60 & $1.459 \mathrm{E}+05$ & $8.871 \mathrm{E}$ & \\
\hline 19 & 1.6911 & 1.60 & 1.80 & $0 E+00$ & 3. & 3. \\
\hline 20 & 1.8907 & 1.80 & 2.00 & $00 \mathrm{E}+00$ & $2.300 \mathrm{E}+03$ & 2. \\
\hline 21 & 2.0900 & 2.00 & 2.20 & $00 E+00$ & $1.302 \mathrm{E}+$ & 1.3 \\
\hline 22 & 2.2892 & 2.20 & 2.40 & $0.000 \mathrm{E}+00$ & $6.991 \mathrm{E}+02$ & $E+0$ \\
\hline 23 & 2.4879 & 2.40 & 2.60 & $00 \mathrm{E}+00$ & $3.482 \mathrm{E}+02$ & +0 \\
\hline 24 & 2.6855 & 2.60 & 2.80 & $00 \mathrm{E}+00$ & $50 \mathrm{E}+0 ?$ & $50 \mathrm{E}+02$ \\
\hline 25 & & 2.80 & 3.20 & $00 \mathrm{E}+00$ & & $=01$ \\
\hline 26 & 3.2000 & 3.20 & 4.00 & $0.000 \mathrm{E}+00$ & $5.883 \mathrm{E}+00$ & $5.883 \mathrm{E}+00$ \\
\hline
\end{tabular}

Total Photons/sec:

$6.782 \mathrm{E}+06 \quad 4.240 \mathrm{E}+06$

1. $102 \mathrm{E}+07$ 
HNF-SD-WM-CN-091

Rev. 0

Page 30 of 64

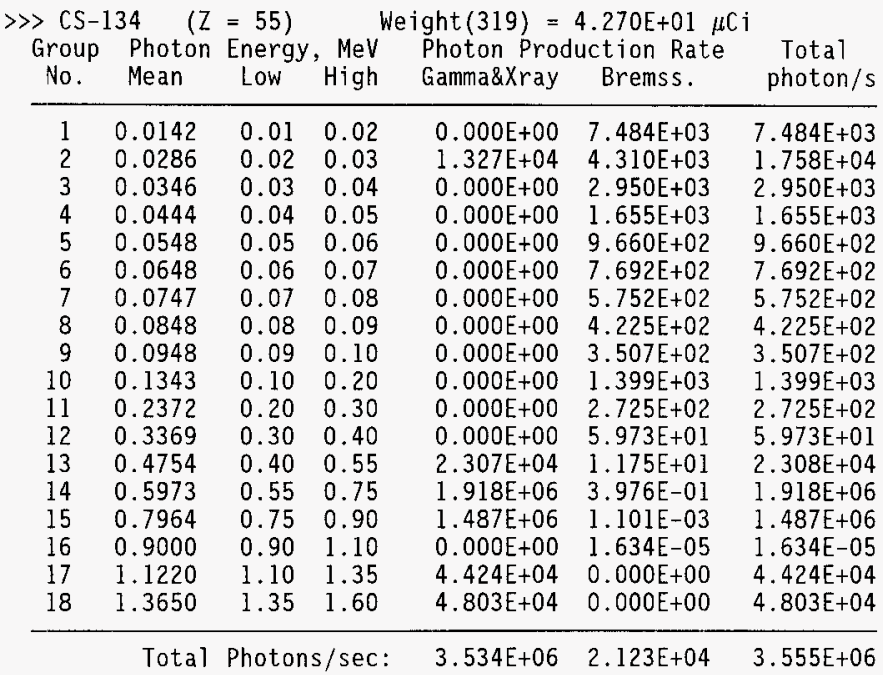

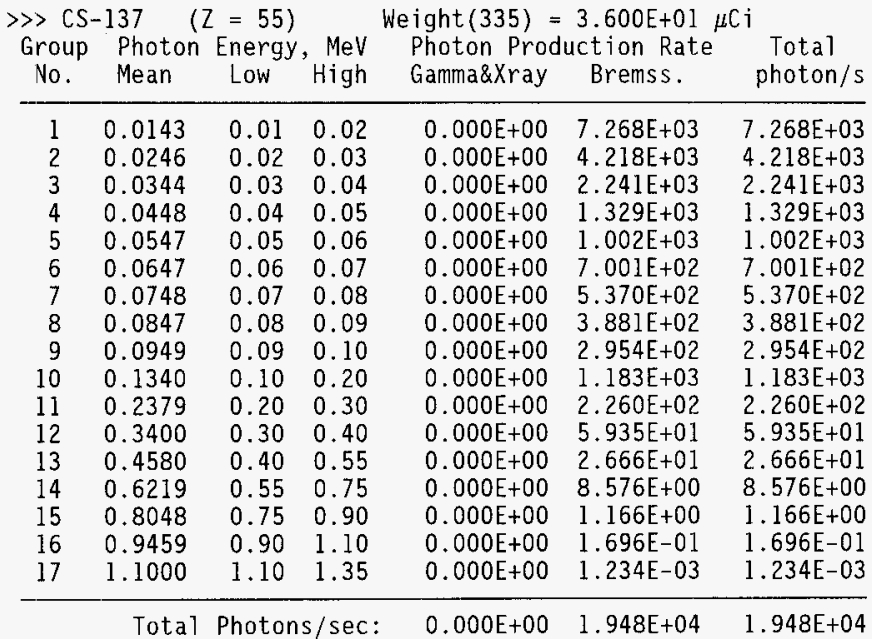


HNF-SD-WM-CN-091

Rev. 0

Page 31 of 64

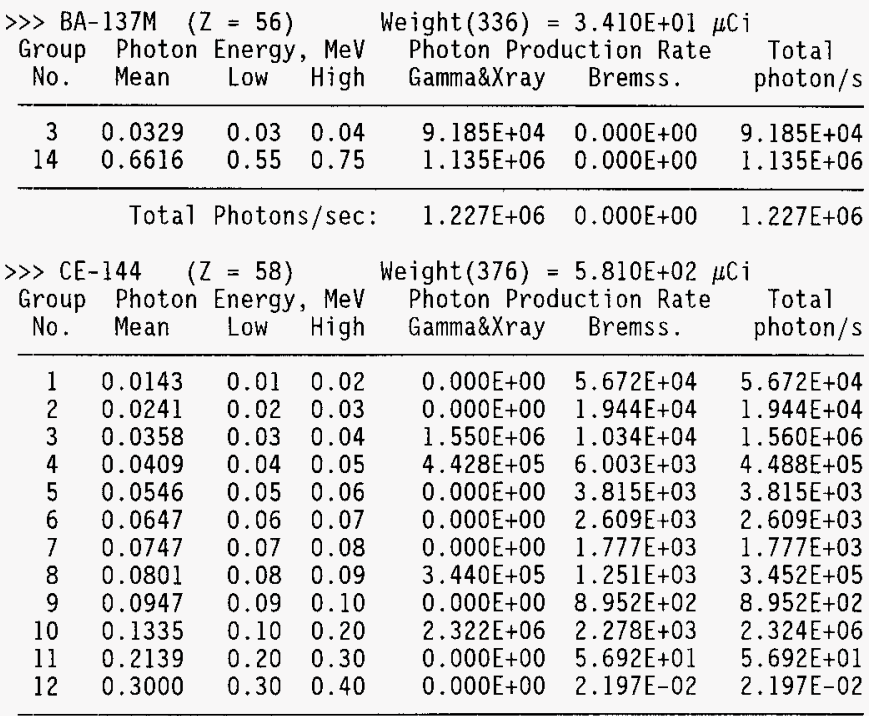

Total Photons/sec: $\quad 4.658 \mathrm{E}+06 \quad 1.052 \mathrm{E}+05 \quad 4.764 \mathrm{E}+06$ 
HNF-SD-WM-CN-091

Rev. 0

Page 32 of 64

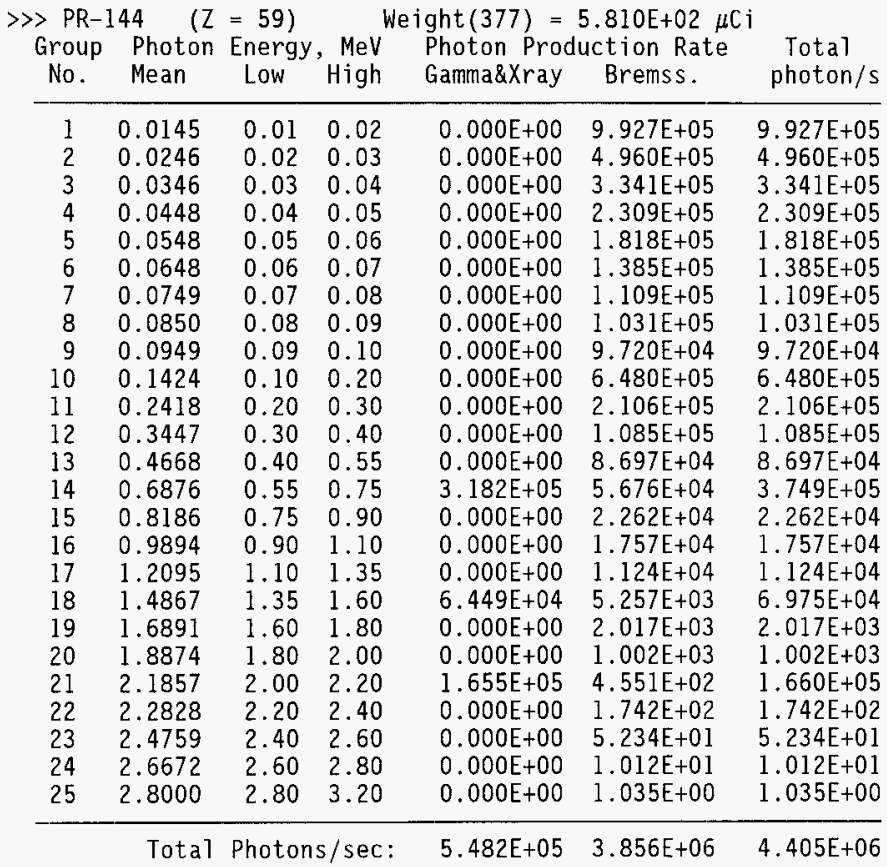

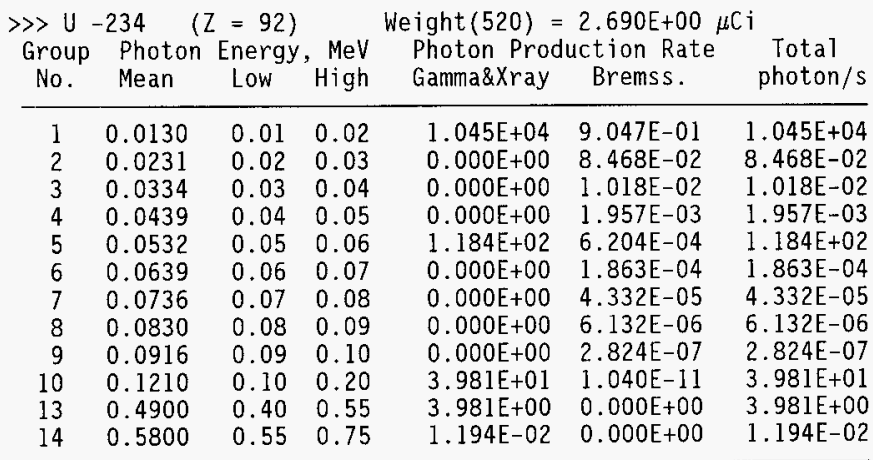

Total Photons/sec: $\quad 1.061 \mathrm{E}+04 \quad 1.002 \mathrm{E}+00 \quad 1.061 \mathrm{E}+04$ 
HNF-SD-WM-CN-091

Rev. 0

Page 33 of 64

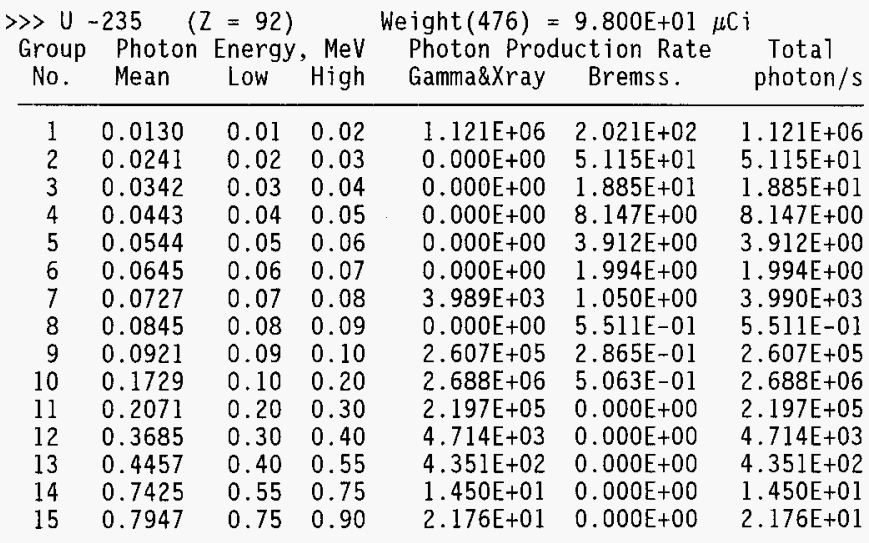

Total Photons/sec: $\quad 4.298 E+06 \quad 2.885 E+02 \quad 4.298 E+06$

\begin{tabular}{ccccccc}
$\begin{array}{c}\text { >> U } \\
\text { Group } \\
\text { No. }\end{array}$ & $\begin{array}{c}\text { Photon Energy, MeV } \\
\text { Mean }\end{array}$ & Low & High & $\begin{array}{c}\text { Weight }(398)=2.170 \mathrm{E}+01 \mu \mathrm{C} i \\
\text { Photon Production Rate } \\
\text { Gamma\&Xray }\end{array}$ & $\begin{array}{c}\text { Botal } \\
\text { Bremss. }\end{array}$ & photon/s \\
\hline 1 & 0.0128 & 0.01 & 0.02 & $0.000 \mathrm{E}+00$ & $4.695 \mathrm{E}+00$ & $4.695 \mathrm{E}+00$ \\
2 & 0.0230 & 0.02 & 0.03 & $0.000 \mathrm{E}+00$ & $3.894 \mathrm{E}-01$ & $3.894 \mathrm{E}-01$ \\
3 & 0.0335 & 0.03 & 0.04 & $0.000 \mathrm{E}+00$ & $4.566 \mathrm{E}-02$ & $4.566 \mathrm{E}-02$ \\
4 & 0.0440 & 0.04 & 0.05 & $0.000 \mathrm{E}+00$ & $1.116 \mathrm{E}-02$ & $1.116 \mathrm{E}-02$ \\
5 & 0.0539 & 0.05 & 0.06 & $0.000 \mathrm{E}+00$ & $3.330 \mathrm{E}-03$ & $3.330 \mathrm{E}-03$ \\
6 & 0.0682 & 0.06 & 0.07 & $8.832 \mathrm{E}+02$ & $7.981 \mathrm{E}-04$ & $8.832 \mathrm{E}+02$ \\
7 & 0.0731 & 0.07 & 0.08 & $0.000 \mathrm{E}+00$ & $1.222 \mathrm{E}-04$ & $1.222 \mathrm{E}-04$ \\
8 & 0.0817 & 0.08 & 0.09 & $0.000 \mathrm{E}+00$ & $6.494 \mathrm{E}-06$ & $6.494 \mathrm{E}-06$ \\
9 & 0.0900 & 0.09 & 0.10 & $0.000 \mathrm{E}+00$ & $3.386 \mathrm{E}-09$ & $3.386 \mathrm{E}-09$ \\
\hline
\end{tabular}

Total Photons/sec: $8.832 E+02 \quad 5.146 E+00 \quad 8.883 E+02$ 
HNF-SD-WM-CN-091

Rev. 0

Page 34 of 64

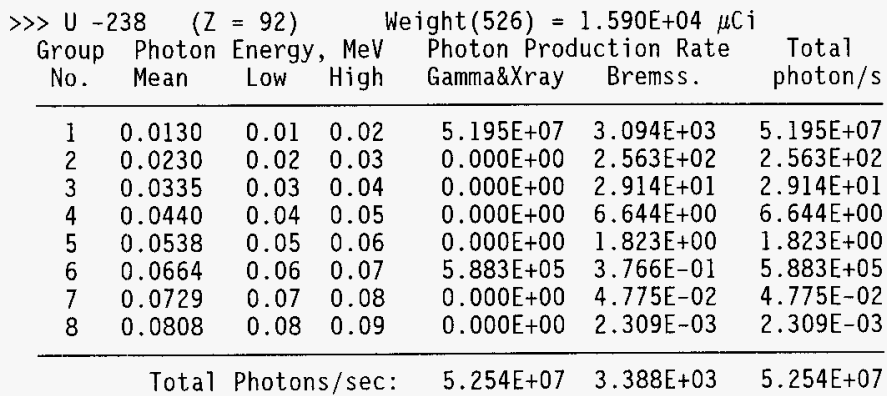


HNF-SD-WM-CN-091

Rev. 0

Page 35 of 64

0ld UNH Tank (HO-64-5920) Line Source - Middle

Shield Composition, $g / c c$

Shield 1 Shield 2 Shield 3

\begin{tabular}{|c|c|c|c|}
\hline $\begin{array}{l}\text { AIR } \\
\text { ALUMINUM } \\
\text { IRON }\end{array}$ & $\begin{array}{l}0.000 \mathrm{E}+00 \\
1.665 \mathrm{E}+00 \\
0.000 \mathrm{E}+00\end{array}$ & $\begin{array}{l}0.000 E+00 \\
0.000 E+00 \\
7.800 E+00\end{array}$ & $\begin{array}{l}1.290 \mathrm{E}-03 \\
0.000 \mathrm{E}+00 \\
0.000 \mathrm{E}+00\end{array}$ \\
\hline Totals: & $1.665 \mathrm{E}+00$ & $7.800 E+00$ & $1.290 \mathrm{E}-03$ \\
\hline $\mathrm{E}, \mathrm{MeV}$ & \multicolumn{3}{|c|}{ Linear Attenuation, per $\mathrm{cm}$ (last } \\
\hline $\begin{array}{l}0.0131 \\
0.0245 \\
0.0354 \\
0.0429 \\
0.0548 \\
0.0659 \\
0.0748 \\
0.0819 \\
0.0933 \\
0.1522 \\
0.2310 \\
0.3458 \\
0.5068 \\
0.6423 \\
0.7680 \\
1.0434 \\
1.1417 \\
1.4187 \\
1.6904 \\
1.8897 \\
2.1850 \\
2.2879 \\
2.4864 \\
2.6844 \\
2.9209 \\
3.2000\end{array}$ & $\begin{array}{l}2.004 \mathrm{E}+01 \\
3.229 \mathrm{E}+00 \\
1.236 \mathrm{E}+00 \\
8.096 \mathrm{E}-01 \\
5.145 \mathrm{E}-01 \\
4.008 \mathrm{E}-01 \\
3.516 \mathrm{E}-01 \\
3.254 \mathrm{E}-01 \\
2.947 \mathrm{E}-01 \\
2.284 \mathrm{E}-01 \\
1.920 \mathrm{E}-01 \\
1.636 \mathrm{E}-01 \\
1.399 \mathrm{E}-01 \\
1.259 \mathrm{E}-01 \\
1.160 \mathrm{E}-01 \\
1.001 \mathrm{E}-01 \\
9.565 \mathrm{E}-02 \\
8.565 \mathrm{E}-02 \\
7.831 \mathrm{E}-02 \\
7.401 \mathrm{E}-02 \\
6.883 \mathrm{E}-02 \\
6.728 \mathrm{E}-02 \\
6.458 \mathrm{E}-02 \\
6.221 \mathrm{E}-02 \\
5.971 \mathrm{E}-02 \\
5.717 \mathrm{E}-02\end{array}$ & $\begin{array}{l}6.779 \mathrm{E}+02 \\
1.146 \mathrm{E}+02 \\
4.037 \mathrm{E}+01 \\
2.318 \mathrm{E}+01 \\
1.182 \mathrm{E}+01 \\
7.416 \mathrm{E}+00 \\
5.442 \mathrm{E}+00 \\
4.420 \mathrm{E}+00 \\
3.335 \mathrm{E}+00 \\
1.508 \mathrm{E}+00 \\
1.031 \mathrm{E}+00 \\
7.918 \mathrm{E}-01 \\
6.509 \mathrm{E}-01 \\
5.802 \mathrm{E}-01 \\
5.316 \mathrm{E}-01 \\
4.565 \mathrm{E}-01 \\
4.360 \mathrm{E}-01 \\
3.906 \mathrm{E}-01 \\
3.585 \mathrm{E}-01 \\
3.402 \mathrm{E}-01 \\
3.188 \mathrm{E}-01 \\
3.124 \mathrm{E}-01 \\
3.017 \mathrm{E}-01 \\
2.926 \mathrm{E}-01 \\
2.836 \mathrm{E}-01 \\
2.751 \mathrm{E}-01\end{array}$ & $\begin{array}{l}2.838 \mathrm{E}-03 \\
6.409 \mathrm{E}-04 \\
3.627 \mathrm{E}-04 \\
2.979 \mathrm{E}-04 \\
2.497 \mathrm{E}-04 \\
2.302 \mathrm{E}-04 \\
2.186 \mathrm{E}-04 \\
2.117 \mathrm{E}-04 \\
2.030 \mathrm{E}-04 \\
1.749 \mathrm{E}-04 \\
1.518 \mathrm{E}-04 \\
1.304 \mathrm{E}-04 \\
1.114 \mathrm{E}-04 \\
1.006 \mathrm{E}-04 \\
9.281 \mathrm{E}-05 \\
8.021 \mathrm{E}-05 \\
7.665 \mathrm{E}-05 \\
6.855 \mathrm{E}-05 \\
6.267 \mathrm{E}-05 \\
5.906 \mathrm{E}-05 \\
5.431 \mathrm{E}-05 \\
5.280 \mathrm{E}-05 \\
5.019 \mathrm{E}-05 \\
4.795 \mathrm{E}-05 \\
4.577 \mathrm{E}-05 \\
4.383 \mathrm{E}-05\end{array}$ \\
\hline
\end{tabular}


HNF-SD-WM-CN-091

Rev. 0

Page 36 of 64

0ld UNH Tank (H0-64-5920) Line Source - Middle

Source Shields Distance to Detector, $X=2.000 \mathrm{E}+00 \mathrm{~cm}$

Cylindrical Cylindrical Source Volume $=2.202 \mathrm{E}+03 \mathrm{cC}$

Source Mass $=3.667 \mathrm{E}+03$ grams

Source Length $=7.010 \mathrm{E}+02 \mathrm{~cm} \quad$ Distance Along Cylinder, $Y=3.505 \mathrm{E}+02 \mathrm{~cm}$

Integration Specs: NTHETA $=11 \quad$ NPSI $=11$ DELR computed internally

Total Intervals: $1.038 \mathrm{E}+05$ (photon source is the lst region)

Shield Thickness: $1.000 \mathrm{E}+00,6.350 \mathrm{E}-01,3.650 \mathrm{E}-01 \mathrm{~cm}$

Distances from Dose Point to the Outside of

(1) Source Region: $1.000 \mathrm{E}+00 \mathrm{~cm}$ (2) Next Layer: $3.650 \mathrm{E}-01 \mathrm{~cm}$

Dose Buildup Data for Shield 2 with Effective Atomic Number 26.00

Buildup Material is Iron

Source values are interpreted as $\mu \mathrm{Ci}$

Source Scale Factor was $1.000 \mathrm{E}+03$

Fluence-to-Dose Conversion: Photons in Air

\begin{tabular}{ccccc}
$\begin{array}{c}\text { Average } \\
\text { E, MeV }\end{array}$ & $\begin{array}{c}\text { Source Total } \\
\text { photons/sec }\end{array}$ & $\begin{array}{c}\text { Fluence to } \\
\text { Dose Factor }\end{array}$ & $\begin{array}{c}\text { Energy Fluence } \\
M e V / \mathrm{cm}^{2} / \mathrm{s}\end{array}$ & $\begin{array}{c}\text { Dose Rate } \\
\text { R/hr }\end{array}$ \\
\hline 0.0131 & $5.519 \mathrm{E}+07$ & $1.263 \mathrm{E}-04$ & $0.000 \mathrm{E}+00$ & $0.000 \mathrm{E}+00$ \\
0.0245 & $1.103 \mathrm{E}+06$ & $1.754 \mathrm{E}-05$ & $0.000 \mathrm{E}+00$ & $0.000 \mathrm{E}+00$ \\
0.0354 & $2.358 \mathrm{E}+06$ & $5.931 \mathrm{E}-06$ & $3.467 \mathrm{E}-12$ & $2.056 \mathrm{E}-17$ \\
0.0429 & $9.431 \mathrm{E}+05$ & $3.621 \mathrm{E}-06$ & $2.045 \mathrm{E}-07$ & $7.406 \mathrm{E}-13$ \\
0.0548 & $3.898 \mathrm{E}+05$ & $2.232 \mathrm{E}-06$ & $3.504 \mathrm{E}-04$ & $7.821 \mathrm{E}-10$ \\
0.0659 & $8.923 \mathrm{E}+05$ & $1.811 \mathrm{E}-06$ & $2.639 \mathrm{E}-02$ & $4.781 \mathrm{E}-08$ \\
0.0748 & $2.460 \mathrm{E}+05$ & $1.667 \mathrm{E}-06$ & $3.775 \mathrm{E}-02$ & $6.292 \mathrm{E}-08$ \\
0.0819 & $5.570 \mathrm{E}+05$ & $1.621 \mathrm{E}-06$ & $2.087 \mathrm{E}-01$ & $3.382 \mathrm{E}-07$ \\
0.0933 & $4.569 \mathrm{E}+05$ & $1.594 \mathrm{E}-06$ & $4.651 \mathrm{E}-01$ & $7.413 \mathrm{E}-07$ \\
0.1522 & $6.358 \mathrm{E}+06$ & $1.734 \mathrm{E}-06$ & $7.600 \mathrm{E}+01$ & $1.318 \mathrm{E}-04$ \\
0.2310 & $7.047 \mathrm{E}+05$ & $1.901 \mathrm{E}-06$ & $1.680 \mathrm{E}+01$ & $3.195 \mathrm{E}-05$ \\
0.3458 & $2.452 \mathrm{E}+05$ & $2.016 \mathrm{E}-06$ & $1.003 \mathrm{E}+01$ & $2.021 \mathrm{E}-05$ \\
0.5068 & $5.574 \mathrm{E}+06$ & $2.040 \mathrm{E}-06$ & $3.606 \mathrm{E}+02$ & $7.356 \mathrm{E}-04$ \\
0.6423 & $6.839 \mathrm{E}+06$ & $2.030 \mathrm{E}-06$ & $5.810 \mathrm{E}+02$ & $1.179 \mathrm{E}-03$ \\
0.7680 & $2.034 \mathrm{E}+07$ & $1.997 \mathrm{E}-06$ & $2.110 \mathrm{E}+03$ & $4.213 \mathrm{E}-03$ \\
1.0434 & $3.834 \mathrm{E}+05$ & $1.907 \mathrm{E}-06$ & $5.536 \mathrm{E}+01$ & $1.056 \mathrm{E}-04$ \\
1.1417 & $1.514 \mathrm{E}+05$ & $1.875 \mathrm{E}-06$ & $2.402 \mathrm{E}+01$ & $4.505 \mathrm{E}-05$ \\
1.4187 & $2.726 \mathrm{E}+05$ & $1.790 \mathrm{E}-06$ & $5.403 \mathrm{E}+01$ & $9.674 \mathrm{E}-05$ \\
1.6904 & $5.942 \mathrm{E}+03$ & $1.714 \mathrm{E}-06$ & $1.403 \mathrm{E}+00$ & $2.406 \mathrm{E}-06$ \\
1.8897 & $3.301 \mathrm{E}+03$ & $1.663 \mathrm{E}-06$ & $8.708 \mathrm{E}-01$ & $1.448 \mathrm{E}-06$ \\
2.1850 & $1.673 \mathrm{E}+05$ & $1.604 \mathrm{E}-06$ & $5.103 \mathrm{E}+01$ & $8.184 \mathrm{E}-05$ \\
2.2879 & $8.734 \mathrm{E}+02$ & $1.593 \mathrm{E}-06$ & $2.790 \mathrm{E}-01$ & $4.445 \mathrm{E}-07$ \\
2.4864 & $4.005 \mathrm{E}+02$ & $1.538 \mathrm{E}-06$ & $1.389 \mathrm{E}-01$ & $2.136 \mathrm{E}-07$ \\
2.6844 & $1.651 \mathrm{E}+02$ & $1.502 \mathrm{E}-06$ & $6.163 \mathrm{E}-02$ & $9.254 \mathrm{E}-08$ \\
2.9209 & $7.672 \mathrm{E}+01$ & $1.465 \mathrm{E}-06$ & $3.102 \mathrm{E}-02$ & $4.543 \mathrm{E}-08$ \\
3.2000 & $5.883 \mathrm{E}+00$ & $1.425 \mathrm{E}-06$ & $2.596 \mathrm{E}-03$ & $3.699 \mathrm{E}-09$ \\
\hline Totals: & $1.032 \mathrm{E}+08$ photons $/ \mathrm{sec}$ & $3.342 \mathrm{E}+03$ & $6.646 \mathrm{E}-03 \mathrm{R} / \mathrm{hr}$
\end{tabular}


HNF-SD-WM-CN-091

Rev. 0

Page 37 of 64

0ld UNH Tank (H0-64-5920) Annular Source - Middle

Shield Composition, g/cc

\begin{tabular}{lllll} 
& Shield 1 & Shield 2 & Shield 3 & Shield 4 \\
\hline AIR & $1.290 \mathrm{E}-03$ & $0.000 \mathrm{E}+00$ & $0.000 \mathrm{E}+00$ & $1.290 \mathrm{E}-03$ \\
ALUMINUM & $0.000 \mathrm{E}+00$ & $1.665 \mathrm{E}+00$ & $0.000 \mathrm{E}+00$ & $0.000 \mathrm{E}+00$ \\
IRON & $0.000 \mathrm{E}+00$ & $0.000 \mathrm{E}+00$ & $7.800 \mathrm{E}+00$ & $0.000 \mathrm{E}+00$ \\
\hline Totals: & $1.290 \mathrm{E}-03$ & $1.665 \mathrm{E}+00$ & $7.800 \mathrm{E}+00$ & $1.290 \mathrm{E}-03$ \\
\hline E, MeV & Linear Attenuation, per cm (1 ast & region is a i $)$ \\
\hline 0.0131 & $2.838 \mathrm{E}-03$ & $2.004 \mathrm{E}+01$ & $6.779 \mathrm{E}+02$ & $2.838 \mathrm{E}-03$ \\
0.0245 & $6.409 \mathrm{E}-04$ & $3.229 \mathrm{E}+00$ & $1.146 \mathrm{E}+02$ & $6.409 \mathrm{E}-04$ \\
0.0354 & $3.627 \mathrm{E}-04$ & $1.236 \mathrm{E}+00$ & $4.037 \mathrm{E}+01$ & $3.627 \mathrm{E}-04$ \\
0.0429 & $2.979 \mathrm{E}-04$ & $8.096 \mathrm{E}-01$ & $2.318 \mathrm{E}+01$ & $2.979 \mathrm{E}-04$ \\
0.0548 & $2.497 \mathrm{E}-04$ & $5.145 \mathrm{E}-01$ & $1.182 \mathrm{E}+01$ & $2.497 \mathrm{E}-04$ \\
0.0659 & $2.302 \mathrm{E}-04$ & $4.008 \mathrm{E}-01$ & $7.416 \mathrm{E}+00$ & $2.302 \mathrm{E}-04$ \\
0.0748 & $2.186 \mathrm{E}-04$ & $3.516 \mathrm{E}-01$ & $5.442 \mathrm{E}+00$ & $2.186 \mathrm{E}-04$ \\
0.0819 & $2.117 \mathrm{E}-04$ & $3.254 \mathrm{E}-01$ & $4.420 \mathrm{E}+00$ & $2.117 \mathrm{E}-04$ \\
0.0933 & $2.030 \mathrm{E}-04$ & $2.947 \mathrm{E}-01$ & $3.335 \mathrm{E}+00$ & $2.030 \mathrm{E}-04$ \\
0.1522 & $1.749 \mathrm{E}-04$ & $2.284 \mathrm{E}-01$ & $1.508 \mathrm{E}+00$ & $1.749 \mathrm{E}-04$ \\
0.2310 & $1.518 \mathrm{E}-04$ & $1.920 \mathrm{E}-01$ & $1.031 \mathrm{E}+00$ & $1.518 \mathrm{E}-04$ \\
0.3458 & $1.304 \mathrm{E}-04$ & $1.636 \mathrm{E}-01$ & $7.918 \mathrm{E}-01$ & $1.304 \mathrm{E}-04$ \\
0.5068 & $1.114 \mathrm{E}-04$ & $1.399 \mathrm{E}-01$ & $6.509 \mathrm{E}-01$ & $1.114 \mathrm{E}-04$ \\
0.6423 & $1.006 \mathrm{E}-04$ & $1.259 \mathrm{E}-01$ & $5.802 \mathrm{E}-01$ & $1.006 \mathrm{E}-04$ \\
0.7680 & $9.281 \mathrm{E}-05$ & $1.160 \mathrm{E}-01$ & $5.316 \mathrm{E}-01$ & $9.281 \mathrm{E}-05$ \\
1.0434 & $8.021 \mathrm{E}-05$ & $1.001 \mathrm{E}-01$ & $4.565 \mathrm{E}-01$ & $8.021 \mathrm{E}-05$ \\
1.1417 & $7.665 \mathrm{E}-05$ & $9.565 \mathrm{E}-02$ & $4.360 \mathrm{E}-01$ & $7.665 \mathrm{E}-05$ \\
1.4187 & $6.855 \mathrm{E}-05$ & $8.565 \mathrm{E}-02$ & $3.906 \mathrm{E}-01$ & $6.855 \mathrm{E}-05$ \\
1.6904 & $6.267 \mathrm{E}-05$ & $7.831 \mathrm{E}-02$ & $3.585 \mathrm{E}-01$ & $6.267 \mathrm{E}-05$ \\
1.8897 & $5.906 \mathrm{E}-05$ & $7.401 \mathrm{E}-02$ & $3.402 \mathrm{E}-01$ & $5.906 \mathrm{E}-05$ \\
2.1850 & $5.431 \mathrm{E}-05$ & $6.883 \mathrm{E}-02$ & $3.188 \mathrm{E}-01$ & $5.431 \mathrm{E}-05$ \\
2.2879 & $5.280 \mathrm{E}-05$ & $6.728 \mathrm{E}-02$ & $3.124 \mathrm{E}-01$ & $5.280 \mathrm{E}-05$ \\
2.4864 & $5.019 \mathrm{E}-05$ & $6.458 \mathrm{E}-02$ & $3.017 \mathrm{E}-01$ & $5.019 \mathrm{E}-05$ \\
2.6844 & $4.795 \mathrm{E}-05$ & $6.221 \mathrm{E}-02$ & $2.926 \mathrm{E}-01$ & $4.795 \mathrm{E}-05$ \\
2.9209 & $4.577 \mathrm{E}-05$ & $5.971 \mathrm{E}-02$ & $2.836 \mathrm{E}-01$ & $4.577 \mathrm{E}-05$ \\
3.2000 & $4.383 \mathrm{E}-05$ & $5.717 \mathrm{E}-02$ & $2.751 \mathrm{E}-01$ & $4.383 \mathrm{E}-05$ \\
\hline & & & &
\end{tabular}


0ld UNH Tank (H0-64-5920) Annular Source - Middle

Source Shields Distance to Detector, $X=7.350 E+01 \mathrm{~cm}$

Annular $13 \quad$ Cyl. \& Slab Source Volume $=3.191 \mathrm{E}+04 \mathrm{cc}$

Source Mass $=5.313 \mathrm{E}+04$ grams

Source Length $=7.010 E+02 \mathrm{~cm} \quad$ Distance Along $C y T$ inder, $Y=3.505 E+02 \mathrm{~cm}$

Integration Specs: $\quad$ NTHETA $=11 \quad$ NPSI $=11 \quad$ DELR computed interna11y

Total Intervals: $1.861 \mathrm{E}+02$ (photon source is the 2 nd region)

Shield Thickness: $7.240 \mathrm{E}+01,1.000 \mathrm{E}-01,6.350 \mathrm{E}-01,3.650 \mathrm{E}-01 \mathrm{~cm}$

Distances from Dose Point to the Outside of

(1) Source Region: $1.000 \mathrm{E}+00 \mathrm{~cm}$ (2) Next Layer: $3.650 \mathrm{E}-01 \mathrm{~cm}$

Dose Buildup Data for Shield 3 with Effective Atomic Number 26.00

Buildup Material is Iron

Source values are interpreted as $\mu \mathrm{C} i$

Source Scale Factor was $1.000 \mathrm{E}+03$

Fluence-to-Dose Conversion: Photons in Air

\begin{tabular}{|c|c|c|c|c|}
\hline $\begin{array}{l}\text { Average } \\
\text { E, MeV }\end{array}$ & $\begin{array}{r}\text { Source Total } \\
\text { photons/sec }\end{array}$ & $\begin{array}{l}\text { Fluence to } \\
\text { Dose Factor }\end{array}$ & $\begin{array}{c}\text { Energy Fluence } \\
\mathrm{MeV} / \mathrm{cm}^{2} / \mathrm{s}\end{array}$ & $\begin{array}{c}\text { Dose Rate } \\
\text { R/hr }\end{array}$ \\
\hline $\begin{array}{l}0.0131 \\
0.0245 \\
0.0354 \\
0.0429 \\
0.0548 \\
0.0659 \\
0.0748 \\
0.0819 \\
0.0933 \\
0.1522 \\
0.2310 \\
0.3458 \\
0.5068 \\
0.6423 \\
0.7680 \\
1.0434 \\
1.1417 \\
1.4187 \\
1.6904 \\
1.8897 \\
2.1850 \\
2.2879 \\
2.4864 \\
2.6844 \\
2.9209 \\
3.2000\end{array}$ & $\begin{array}{l}5.519 \mathrm{E}+07 \\
1.103 \mathrm{E}+06 \\
2.358 \mathrm{E}+06 \\
9.431 \mathrm{E}+05 \\
3.898 \mathrm{E}+05 \\
8.923 \mathrm{E}+05 \\
2.460 \mathrm{E}+05 \\
5.570 \mathrm{E}+05 \\
4.569 \mathrm{E}+05 \\
6.358 \mathrm{E}+06 \\
7.047 \mathrm{E}+05 \\
2.452 \mathrm{E}+05 \\
5.574 \mathrm{E}+06 \\
6.839 \mathrm{E}+06 \\
2.034 \mathrm{E}+07 \\
3.834 \mathrm{E}+05 \\
1.514 \mathrm{E}+05 \\
2.726 \mathrm{E}+05 \\
5.942 \mathrm{E}+03 \\
3.301 \mathrm{E}+03 \\
1.673 \mathrm{E}+05 \\
8.734 \mathrm{E}+02 \\
4.005 \mathrm{E}+02 \\
1.651 \mathrm{E}+02 \\
7.672 \mathrm{E}+01 \\
5.883 \mathrm{E}+00\end{array}$ & $\begin{array}{l}1.263 \mathrm{E}-04 \\
1.754 \mathrm{E}-05 \\
5.931 \mathrm{E}-06 \\
3.621 \mathrm{E}-06 \\
2.232 \mathrm{E}-06 \\
1.811 \mathrm{E}-06 \\
1.667 \mathrm{E}-06 \\
1.621 \mathrm{E}-06 \\
1.594 \mathrm{E}-06 \\
1.734 \mathrm{E}-06 \\
1.901 \mathrm{E}-06 \\
2.016 \mathrm{E}-06 \\
2.040 \mathrm{E}-06 \\
2.030 \mathrm{E}-06 \\
1.997 \mathrm{E}-06 \\
1.907 \mathrm{E}-06 \\
1.875 \mathrm{E}-06 \\
1.790 \mathrm{E}-06 \\
1.714 \mathrm{E}-06 \\
1.663 \mathrm{E}-06 \\
1.604 \mathrm{E}-06 \\
1.593 \mathrm{E}-06 \\
1.538 \mathrm{E}-06 \\
1.502 \mathrm{E}-06 \\
1.465 \mathrm{E}-06 \\
1.425 \mathrm{E}-06\end{array}$ & $\begin{array}{l}0.000 \mathrm{E}+00 \\
0.000 \mathrm{E}+00 \\
9.045 \mathrm{E}-14 \\
4.337 \mathrm{E}-09 \\
6.550 \mathrm{E}-06 \\
4.899 \mathrm{E}-04 \\
7.203 \mathrm{E}-04 \\
4.101 \mathrm{E}-03 \\
9.633 \mathrm{E}-03 \\
1.972 \mathrm{E}+00 \\
4.817 \mathrm{E}-01 \\
3.055 \mathrm{E}-01 \\
1.143 \mathrm{E}+01 \\
1.880 \mathrm{E}+01 \\
6.927 \mathrm{E}+01 \\
1.860 \mathrm{E}+00 \\
8.121 \mathrm{E}-01 \\
1.851 \mathrm{E}+00 \\
4.848 \mathrm{E}-02 \\
3.023 \mathrm{E}-02 \\
1.782 \mathrm{E}+00 \\
9.756 \mathrm{E}-03 \\
4.869 \mathrm{E}-03 \\
2.163 \mathrm{E}-03 \\
1.090 \mathrm{E}-03 \\
9.126 \mathrm{E}-05\end{array}$ & $\begin{array}{l}0.000 \mathrm{E}+00 \\
0.000 \mathrm{E}+00 \\
5.364 \mathrm{E}-19 \\
1.570 \mathrm{E}-14 \\
1.462 \mathrm{E}-11 \\
8.874 \mathrm{E}-10 \\
1.201 \mathrm{E}-09 \\
6.647 \mathrm{E}-09 \\
1.535 \mathrm{E}-08 \\
3.419 \mathrm{E}-06 \\
9.158 \mathrm{E}-07 \\
6.158 \mathrm{E}-07 \\
2.331 \mathrm{E}-05 \\
3.815 \mathrm{E}-05 \\
1.383 \mathrm{E}-04 \\
3.548 \mathrm{E}-06 \\
1.523 \mathrm{E}-06 \\
3.314 \mathrm{E}-06 \\
8.312 \mathrm{E}-08 \\
5.026 \mathrm{E}-08 \\
2.858 \mathrm{E}-06 \\
1.554 \mathrm{E}-08 \\
7.487 \mathrm{E}-09 \\
3.249 \mathrm{E}-09 \\
1.596 \mathrm{E}-09 \\
1.300 \mathrm{E}-10\end{array}$ \\
\hline
\end{tabular}

Totals: $1.032 \mathrm{E}+08$ photons $/ \mathrm{sec} \quad 1.087 \mathrm{E}+02 \quad 2.161 \mathrm{E}-04 \mathrm{R} / \mathrm{hr}$ 
HNF-SD-WM-CN-091

Rev. 0

Page 39 of 64

01d UNH Tank (H0-64-5920) Line Source - End

Shield Composition, $g / c c$

Shield 1 Shield 2 Shield 3

\begin{tabular}{|c|c|c|c|}
\hline $\begin{array}{l}\text { AIR } \\
\text { ALUMINUM } \\
\text { IRON }\end{array}$ & $\begin{array}{l}0.000 E+00 \\
1.665 E+00 \\
0.000 E+00\end{array}$ & $\begin{array}{l}0.000 E+00 \\
0.000 E+00 \\
7.800 E+00\end{array}$ & $\begin{array}{l}1.290 E-03 \\
0.000 E+00 \\
0.000 E+00\end{array}$ \\
\hline Totals: & $1.665 \mathrm{E}+00$ & $7.800 \mathrm{E}+00$ & $1.290 \mathrm{E}-03$ \\
\hline $\mathrm{E}, \mathrm{MeV}$ & \multicolumn{3}{|c|}{ Linear Attenuation, per cm (last } \\
\hline $\begin{array}{l}0.0131 \\
0.0245 \\
0.0354 \\
0.0429 \\
0.0548 \\
0.0659 \\
0.0748 \\
0.0819 \\
0.0933 \\
0.1522 \\
0.2310 \\
0.3458 \\
0.5068 \\
0.6423 \\
0.7680 \\
1.0434 \\
1.1417 \\
1.4187 \\
1.6904 \\
1.8897 \\
2.1850 \\
2.2879 \\
2.4864 \\
2.6844 \\
2.9209 \\
3.2000\end{array}$ & $\begin{array}{l}2.004 \mathrm{E}+01 \\
3.229 \mathrm{E}+00 \\
1.236 \mathrm{E}+00 \\
8.096 \mathrm{E}-01 \\
5.145 \mathrm{E}-01 \\
4.008 \mathrm{E}-01 \\
3.516 \mathrm{E}-01 \\
3.254 \mathrm{E}-01 \\
2.947 \mathrm{E}-01 \\
2.284 \mathrm{E}-01 \\
1.920 \mathrm{E}-01 \\
1.636 \mathrm{E}-01 \\
1.399 \mathrm{E}-01 \\
1.259 \mathrm{E}-01 \\
1.160 \mathrm{E}-01 \\
1.001 \mathrm{E}-01 \\
9.565 \mathrm{E}-02 \\
8.565 \mathrm{E}-02 \\
7.831 \mathrm{E}-02 \\
7.401 \mathrm{E}-02 \\
6.883 \mathrm{E}-02 \\
6.728 \mathrm{E}-02 \\
6.458 \mathrm{E}-02 \\
6.221 \mathrm{E}-02 \\
5.971 \mathrm{E}-02 \\
5.717 \mathrm{E}-02\end{array}$ & $\begin{array}{l}6.779 \mathrm{E}+02 \\
1.146 \mathrm{E}+02 \\
4.037 \mathrm{E}+01 \\
2.318 \mathrm{E}+01 \\
1.182 \mathrm{E}+01 \\
7.416 \mathrm{E}+00 \\
5.442 \mathrm{E}+00 \\
4.420 \mathrm{E}+00 \\
3.335 \mathrm{E}+00 \\
1.508 \mathrm{E}+00 \\
1.031 \mathrm{E}+00 \\
7.918 \mathrm{E}-01 \\
6.509 \mathrm{E}-01 \\
5.802 \mathrm{E}-01 \\
5.316 \mathrm{E}-01 \\
4.565 \mathrm{E}-01 \\
4.360 \mathrm{E}-01 \\
3.906 \mathrm{E}-01 \\
3.585 \mathrm{E}-01 \\
3.402 \mathrm{E}-01 \\
3.188 \mathrm{E}-01 \\
3.124 \mathrm{E}-01 \\
3.017 \mathrm{E}-01 \\
2.926 \mathrm{E}-01 \\
2.836 \mathrm{E}-01 \\
2.751 \mathrm{E}-01\end{array}$ & $\begin{array}{l}2.838 \mathrm{E}-03 \\
6.409 \mathrm{E}-04 \\
3.627 \mathrm{E}-04 \\
2.979 \mathrm{E}-04 \\
2.497 \mathrm{E}-04 \\
2.302 \mathrm{E}-04 \\
2.186 \mathrm{E}-04 \\
2.117 \mathrm{E}-04 \\
2.030 \mathrm{E}-04 \\
1.749 \mathrm{E}-04 \\
1.518 \mathrm{E}-04 \\
1.304 \mathrm{E}-04 \\
1.114 \mathrm{E}-04 \\
1.006 \mathrm{E}-04 \\
9.281 \mathrm{E}-05 \\
8.021 \mathrm{E}-05 \\
7.665 \mathrm{E}-05 \\
5.855 \mathrm{E}-05 \\
6.267 \mathrm{E}-05 \\
5.906 \mathrm{E}-05 \\
5.431 \mathrm{E}-05 \\
5.280 \mathrm{E}-05 \\
5.019 \mathrm{E}-05 \\
4.795 \mathrm{E}-05 \\
4.577 \mathrm{E}-05 \\
4.383 \mathrm{E}-05\end{array}$ \\
\hline
\end{tabular}


HNF-SD-WM-CN-091

Rev. 0

Page 40 of 64

0ld UNH Tank (H0-64-5920) Line Source - End

Source Shields Distance to Detector, $X=2.000 \mathrm{E}+00 \mathrm{~cm}$

cylindrical

Cyl indrical

Source Volume $=2.202 \mathrm{E}+03 \mathrm{cC}$

Source Mass $=3.667 \mathrm{E}+03$ grams

Source Length $=7.010 E+02 \mathrm{~cm} \quad$ Distance ATong Cyl inder, $Y=7.000 E+02 \mathrm{~cm}$

Integration Specs: $\quad$ NTHETA $=11 \quad$ NPSI $=11$ DELR computed internally

Total Intervals: $1.038 \mathrm{E}+05$ (photon source is the lst region)

Shield Thickness: $1.000 \mathrm{E}+00,6.350 \mathrm{E}-01,3.650 \mathrm{E}-01 \mathrm{~cm}$

Distances from Dose Point to the Outside of

(1) Source Region: $1.000 \mathrm{E}+00 \mathrm{~cm}$ (2) Next Layer: $3.650 \mathrm{E}-01 \mathrm{~cm}$

Dose Buildup Data for Shield 2 with Effective Atomic Number 26.00

Buildup Material is Iron

Source values are interpreted as $\mu \mathrm{Ci}$

Source Scale Factor was $1.000 \mathrm{E}+03$

Fluence-to-Dose Conversion: Photons in Air

\begin{tabular}{ccccc}
$\begin{array}{c}\text { Average } \\
\text { E, MeV }\end{array}$ & $\begin{array}{c}\text { Source Total } \\
\text { photons/sec }\end{array}$ & $\begin{array}{c}\text { Fluence to } \\
\text { Dose Factor }\end{array}$ & $\begin{array}{c}\text { Energy Fluence } \\
\text { MeV } / \mathrm{cm} / \mathrm{s}\end{array}$ & $\begin{array}{c}\text { Dose Rate } \\
\text { R/hr }\end{array}$ \\
\hline 0.0131 & $5.519 \mathrm{E}+07$ & $1.263 \mathrm{E}-04$ & $0.000 \mathrm{E}+00$ & $0.000 \mathrm{E}+00$ \\
0.0245 & $1.103 \mathrm{E}+06$ & $1.754 \mathrm{E}-05$ & $0.000 \mathrm{E}+00$ & $0.000 \mathrm{E}+00$ \\
0.0354 & $2.358 \mathrm{E}+06$ & $5.931 \mathrm{E}-06$ & $3.453 \mathrm{E}-12$ & $2.048 \mathrm{E}-17$ \\
0.0429 & $9.431 \mathrm{E}+05$ & $3.621 \mathrm{E}-06$ & $2.008 \mathrm{E}-07$ & $7.272 \mathrm{E}-13$ \\
0.0548 & $3.898 \mathrm{E}+05$ & $2.232 \mathrm{E}-06$ & $3.329 \mathrm{E}-04$ & $7.431 \mathrm{E}-10$ \\
0.0659 & $8.923 \mathrm{E}+05$ & $1.811 \mathrm{E}-06$ & $2.412 \mathrm{E}-02$ & $4.368 \mathrm{E}-08$ \\
0.0748 & $2.460 \mathrm{E}+05$ & $1.667 \mathrm{E}-06$ & $3.350 \mathrm{E}-02$ & $5.584 \mathrm{E}-08$ \\
0.0819 & $5.570 \mathrm{E}+05$ & $1.621 \mathrm{E}-06$ & $1.813 \mathrm{E}-01$ & $2.937 \mathrm{E}-07$ \\
0.0933 & $4.569 \mathrm{E}+05$ & $1.594 \mathrm{E}-06$ & $3.925 \mathrm{E}-01$ & $6.256 \mathrm{E}-07$ \\
0.1522 & $6.358 \mathrm{E}+06$ & $1.734 \mathrm{E}-06$ & $5.847 \mathrm{E}+01$ & $1.014 \mathrm{E}-04$ \\
0.2310 & $7.047 \mathrm{E}+05$ & $1.901 \mathrm{E}-06$ & $1.256 \mathrm{E}+01$ & $2.388 \mathrm{E}-05$ \\
0.3458 & $2.452 \mathrm{E}+05$ & $2.016 \mathrm{E}-06$ & $7.362 \mathrm{E}+00$ & $1.484 \mathrm{E}-05$ \\
0.5068 & $5.574 \mathrm{E}+06$ & $2.040 \mathrm{E}-06$ & $2.618 \mathrm{E}+02$ & $5.340 \mathrm{E}-04$ \\
0.6423 & $6.839 \mathrm{E}+06$ & $2.030 \mathrm{E}-06$ & $4.193 \mathrm{E}+02$ & $8.509 \mathrm{E}-04$ \\
0.7680 & $2.034 \mathrm{E}+07$ & $1.997 \mathrm{E}-06$ & $1.516 \mathrm{E}+03$ & $3.027 \mathrm{E}-03$ \\
1.0434 & $3.834 \mathrm{E}+05$ & $1.907 \mathrm{E}-06$ & $3.955 \mathrm{E}+01$ & $7.543 \mathrm{E}-05$ \\
1.1417 & $1.514 \mathrm{E}+05$ & $1.875 \mathrm{E}-06$ & $1.714 \mathrm{E}+01$ & $3.213 \mathrm{E}-05$ \\
1.4187 & $2.726 \mathrm{E}+05$ & $1.790 \mathrm{E}-06$ & $3.843 \mathrm{E}+01$ & $6.880 \mathrm{E}-05$ \\
1.6904 & $5.942 \mathrm{E}+03$ & $1.714 \mathrm{E}-06$ & $9.962 \mathrm{E}-01$ & $1.708 \mathrm{E}-06$ \\
1.8897 & $3.301 \mathrm{E}+03$ & $1.663 \mathrm{E}-06$ & $6.178 \mathrm{E}-01$ & $1.027 \mathrm{E}-06$ \\
2.1850 & $1.673 \mathrm{E}+05$ & $1.604 \mathrm{E}-06$ & $3.616 \mathrm{E}+01$ & $5.800 \mathrm{E}-05$ \\
2.2879 & $8.734 \mathrm{E}+02$ & $1.593 \mathrm{E}-06$ & $1.976 \mathrm{E}-01$ & $3.149 \mathrm{E}-07$ \\
2.4864 & $4.005 \mathrm{E}+02$ & $1.538 \mathrm{E}-06$ & $9.835 \mathrm{E}-02$ & $1.512 \mathrm{E}-07$ \\
2.6844 & $1.651 \mathrm{E}+02$ & $1.502 \mathrm{E}-06$ & $4.364 \mathrm{E}-02$ & $6.552 \mathrm{E}-08$ \\
2.9209 & $7.672 \mathrm{E}+01$ & $1.465 \mathrm{E}-06$ & $2.196 \mathrm{E}-02$ & $3.217 \mathrm{E}-08$ \\
3.2000 & $5.883 \mathrm{E}+00$ & $1.425 \mathrm{E}-06$ & $1.838 \mathrm{E}-03$ & $2.619 \mathrm{E}-09$ \\
\hline Tota1s: & $1.032 \mathrm{E}+08$ & photons $/ \mathrm{sec}$ & $2.409 \mathrm{E}+03$ & $4.791 \mathrm{E}-03 \mathrm{R} / \mathrm{hr}$ \\
& & & &
\end{tabular}


HNF-SD-WM-CN-091

Rev. 0

Page 41 of 64

0ld UNH Tank (H0-64-5920) Annular Source - End

Shield Composition, $\mathrm{g} / \mathrm{cc}$

Shield 1 Shield 2 Shield 3 Shield 4

\begin{tabular}{lllll}
\hline AIR & $1.290 \mathrm{E}-03$ & $0.000 \mathrm{E}+00$ & $0.000 \mathrm{E}+00$ & $1.290 \mathrm{E}-03$ \\
ALUMINUM & $0.000 \mathrm{E}+00$ & $1.665 \mathrm{E}+00$ & $0.000 \mathrm{E}+00$ & $0.000 \mathrm{E}+00$ \\
IRON & $0.000 \mathrm{E}+00$ & $0.000 \mathrm{E}+00$ & $7.800 \mathrm{E}+00$ & $0.000 \mathrm{E}+00$ \\
\hline Totals: & $1.290 \mathrm{E}-03$ & $1.665 \mathrm{E}+00$ & $7.800 \mathrm{E}+00$ & $1.290 \mathrm{E}-03$ \\
\hline
\end{tabular}

$\mathrm{E}, \mathrm{MeV}$ Linear Attenuation, per $\mathrm{cm}$ (7ast region is air)

\begin{tabular}{lllll}
0.0131 & $2.838 \mathrm{E}-03$ & $2.004 \mathrm{E}+01$ & $6.779 \mathrm{E}+02$ & $2.838 \mathrm{E}-03$ \\
0.0245 & $6.409 \mathrm{E}-04$ & $3.229 \mathrm{E}+00$ & $1.146 \mathrm{E}+02$ & $6.409 \mathrm{E}-04$ \\
0.0354 & $3.627 \mathrm{E}-04$ & $1.236 \mathrm{E}+00$ & $4.037 \mathrm{E}+01$ & $3.627 \mathrm{E}-04$ \\
0.0429 & $2.979 \mathrm{E}-04$ & $8.096 \mathrm{E}-01$ & $2.318 \mathrm{E}+01$ & $2.979 \mathrm{E}-04$ \\
0.0548 & $2.497 \mathrm{E}-04$ & $5.145 \mathrm{E}-01$ & $1.182 \mathrm{E}+01$ & $2.497 \mathrm{E}-04$ \\
0.0659 & $2.302 \mathrm{E}-04$ & $4.008 \mathrm{E}-01$ & $7.416 \mathrm{E}+00$ & $2.302 \mathrm{E}-04$ \\
0.0748 & $2.186 \mathrm{E}-04$ & $3.516 \mathrm{E}-01$ & $5.442 \mathrm{E}+00$ & $2.186 \mathrm{E}-04$ \\
0.0819 & $2.117 \mathrm{E}-04$ & $3.254 \mathrm{E}-01$ & $4.420 \mathrm{E}+00$ & $2.117 \mathrm{E}-04$ \\
0.0933 & $2.030 \mathrm{E}-04$ & $2.947 \mathrm{E}-01$ & $3.335 \mathrm{E}+00$ & $2.030 \mathrm{E}-04$ \\
0.1522 & $1.749 \mathrm{E}-04$ & $2.284 \mathrm{E}-01$ & $1.508 \mathrm{E}+00$ & $1.749 \mathrm{E}-04$ \\
0.2310 & $1.518 \mathrm{E}-04$ & $1.920 \mathrm{E}-01$ & $1.031 \mathrm{E}+00$ & $1.518 \mathrm{E}-04$ \\
0.3458 & $1.304 \mathrm{E}-04$ & $1.636 \mathrm{E}-01$ & $7.918 \mathrm{E}-01$ & $1.304 \mathrm{E}-04$ \\
0.5068 & $1.114 \mathrm{E}-04$ & $1.399 \mathrm{E}-01$ & $6.509 \mathrm{E}-01$ & $1.114 \mathrm{E}-04$ \\
0.6423 & $1.006 \mathrm{E}-04$ & $1.259 \mathrm{E}-01$ & $5.802 \mathrm{E}-01$ & $1.006 \mathrm{E}-04$ \\
0.7680 & $9.281 \mathrm{E}-05$ & $1.160 \mathrm{E}-01$ & $5.316 \mathrm{E}-01$ & $9.281 \mathrm{E}-05$ \\
1.0434 & $8.021 \mathrm{E}-05$ & $1.001 \mathrm{E}-01$ & $4.565 \mathrm{E}-01$ & $8.021 \mathrm{E}-05$ \\
1.1417 & $7.665 \mathrm{E}-05$ & $9.565 \mathrm{E}-02$ & $4.360 \mathrm{E}-01$ & $7.665 \mathrm{E}-05$ \\
1.4187 & $6.855 \mathrm{E}-05$ & $8.565 \mathrm{E}-02$ & $3.906 \mathrm{E}-01$ & $6.855 \mathrm{E}-05$ \\
1.6904 & $5.267 \mathrm{E}-05$ & $7.831 \mathrm{E}-02$ & $3.585 \mathrm{E}-01$ & $6.267 \mathrm{E}-05$ \\
1.8897 & $5.906 \mathrm{E}-05$ & $7.401 \mathrm{E}-02$ & $3.402 \mathrm{E}-01$ & $5.906 \mathrm{E}-05$ \\
2.1850 & $5.431 \mathrm{E}-05$ & $6.883 \mathrm{E}-02$ & $3.188 \mathrm{E}-01$ & $5.431 \mathrm{E}-05$ \\
2.2879 & $5.280 \mathrm{E}-05$ & $6.728 \mathrm{E}-02$ & $3.124 \mathrm{E}-01$ & $5.280 \mathrm{E}-05$ \\
2.4864 & $5.019 \mathrm{E}-05$ & $6.458 \mathrm{E}-02$ & $3.017 \mathrm{E}-01$ & $5.019 \mathrm{E}-05$ \\
2.6844 & $4.795 \mathrm{E}-05$ & $6.221 \mathrm{E}-02$ & $2.926 \mathrm{E}-01$ & $4.795 \mathrm{E}-05$ \\
2.9209 & $4.577 \mathrm{E}-05$ & $5.971 \mathrm{E}-02$ & $2.836 \mathrm{E}-01$ & $4.577 \mathrm{E}-05$ \\
3.2000 & $4.383 \mathrm{E}-05$ & $5.717 \mathrm{E}-02$ & $2.751 \mathrm{E}-01$ & $4.383 \mathrm{E}-05$ \\
\hline
\end{tabular}




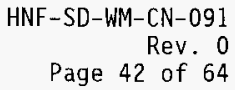

0ld UNH Tank (H0-64-5920) Annular Source - End

Source Shields Distance to Detector, $X=7.350 E+01 \mathrm{~cm}$

Annular $13 \quad C y l$. \& S1ab Source Volume $=3.191 E+04 c c$

Source Mass $=5.313 E+04$ grams

Source Length $=7.010 E+02 \mathrm{~cm} \quad$ Distance Along $C y l$ inder, $Y=7.000 E+02 \mathrm{~cm}$

Integration Specs: $\quad$ NTHETA $=11$ NPSI $=11$ DELR computed internally

Total Intervals: $1.861 \mathrm{E}+02$ (photon source is the 2nd region)

Shield Thickness: $7.240 \mathrm{E}+01,1.000 \mathrm{E}-01,6.350 \mathrm{E}-01,3.650 \mathrm{E}-01 \mathrm{~cm}$

Distances from Dose Point to the Outside of

(1) Source Region: $1.000 \mathrm{E}+00 \mathrm{~cm}$ (2) Next Layer: $3.650 \mathrm{E}-01 \mathrm{~cm}$

Dose Buildup Data for Shield 3 with Effective Atomic Number 26.00

Buildup Material is Iron

Source values are interpreted as $\mu \mathrm{C} \mathbf{i}$

Source Scale Factor was $1.000 E+03$

Fluence-to-Dose Conversion: Photons in Air

\begin{tabular}{ccccc}
$\begin{array}{c}\text { Average } \\
\text { E, MeV }\end{array}$ & $\begin{array}{c}\text { Source Total } \\
\text { photons } / \mathrm{sec}\end{array}$ & $\begin{array}{c}\text { Fluence to } \\
\text { Dose Factor }\end{array}$ & $\begin{array}{c}\text { Energy Fluence } \\
\text { MeV/cm } / \mathrm{s}\end{array}$ & $\begin{array}{c}\text { Dose Rate } \\
\text { R/hr }\end{array}$ \\
\hline 0.0131 & $5.519 \mathrm{E}+07$ & $1.263 \mathrm{E}-04$ & $0.000 \mathrm{E}+00$ & $0.000 \mathrm{E}+00$ \\
0.0245 & $1.103 \mathrm{E}+06$ & $1.754 \mathrm{E}-05$ & $0.000 \mathrm{E}+00$ & $0.000 \mathrm{E}+00$ \\
0.0354 & $2.358 \mathrm{E}+06$ & $5.931 \mathrm{E}-06$ & $7.521 \mathrm{E}-14$ & $4.460 \mathrm{E}-19$ \\
0.0429 & $9.431 \mathrm{E}+05$ & $3.621 \mathrm{E}-06$ & $3.537 \mathrm{E}-09$ & $1.281 \mathrm{E}-14$ \\
0.0548 & $3.898 \mathrm{E}+05$ & $2.232 \mathrm{E}-06$ & $5.219 \mathrm{E}-06$ & $1.165 \mathrm{E}-11$ \\
0.0659 & $8.923 \mathrm{E}+05$ & $1.811 \mathrm{E}-06$ & $3.819 \mathrm{E}-04$ & $6.917 \mathrm{E}-10$ \\
0.0748 & $2.460 \mathrm{E}+05$ & $1.667 \mathrm{E}-06$ & $5.514 \mathrm{E}-04$ & $9.191 \mathrm{E}-10$ \\
0.0819 & $5.570 \mathrm{E}+05$ & $1.621 \mathrm{E}-06$ & $3.097 \mathrm{E}-03$ & $5.019 \mathrm{E}-09$ \\
0.0933 & $4.569 \mathrm{E}+05$ & $1.594 \mathrm{E}-06$ & $7.129 \mathrm{E}-03$ & $1.136 \mathrm{E}-08$ \\
0.1522 & $6.358 \mathrm{E}+06$ & $1.734 \mathrm{E}-06$ & $1.359 \mathrm{E}+00$ & $2.356 \mathrm{E}-06$ \\
0.2310 & $7.047 \mathrm{E}+05$ & $1.901 \mathrm{E}-06$ & $3.242 \mathrm{E}-01$ & $6.164 \mathrm{E}-07$ \\
0.3458 & $2.452 \mathrm{E}+05$ & $2.016 \mathrm{E}-06$ & $2.028 \mathrm{E}-01$ & $4.088 \mathrm{E}-07$ \\
0.5068 & $5.574 \mathrm{E}+06$ & $2.040 \mathrm{E}-06$ & $7.521 \mathrm{E}+00$ & $1.534 \mathrm{E}-05$ \\
0.6423 & $6.839 \mathrm{E}+06$ & $2.030 \mathrm{E}-06$ & $1.232 \mathrm{E}+01$ & $2.501 \mathrm{E}-05$ \\
0.7680 & $2.034 \mathrm{E}+07$ & $1.997 \mathrm{E}-06$ & $4.527 \mathrm{E}+01$ & $9.038 \mathrm{E}-05$ \\
1.0434 & $3.834 \mathrm{E}+05$ & $1.907 \mathrm{E}-06$ & $1.210 \mathrm{E}+00$ & $2.308 \mathrm{E}-06$ \\
1.1417 & $1.514 \mathrm{E}+05$ & $1.875 \mathrm{E}-06$ & $5.278 \mathrm{E}-01$ & $9.897 \mathrm{E}-07$ \\
1.4187 & $2.726 \mathrm{E}+05$ & $1.790 \mathrm{E}-06$ & $1.200 \mathrm{E}+00$ & $2.149 \mathrm{E}-06$ \\
1.6904 & $5.942 \mathrm{E}+03$ & $1.714 \mathrm{E}-06$ & $3.139 \mathrm{E}-02$ & $5.382 \mathrm{E}-08$ \\
1.8897 & $3.301 \mathrm{E}+03$ & $1.663 \mathrm{E}-06$ & $1.956 \mathrm{E}-02$ & $3.252 \mathrm{E}-08$ \\
2.1850 & $1.673 \mathrm{E}+05$ & $1.604 \mathrm{E}-06$ & $1.152 \mathrm{E}+00$ & $1.847 \mathrm{E}-06$ \\
2.2879 & $8.734 \mathrm{E}+02$ & $1.593 \mathrm{E}-06$ & $6.304 \mathrm{E}-03$ & $1.004 \mathrm{E}-08$ \\
2.4864 & $4.005 \mathrm{E}+02$ & $1.538 \mathrm{E}-06$ & $3.145 \mathrm{E}-03$ & $4.836 \mathrm{E}-09$ \\
2.6844 & $1.651 \mathrm{E}+02$ & $1.502 \mathrm{E}-06$ & $1.397 \mathrm{E}-03$ & $2.098 \mathrm{E}-09$ \\
2.9209 & $7.672 \mathrm{E}+01$ & $1.465 \mathrm{E}-06$ & $7.038 \mathrm{E}-04$ & $1.031 \mathrm{E}-09$ \\
3.2000 & $5.883 \mathrm{E}+00$ & $1.425 \mathrm{E}-06$ & $5.892 \mathrm{E}-05$ & $8.395 \mathrm{E}-11$ \\
\hline
\end{tabular}

Totals: $1.032 \mathrm{E}+08$ photons $/ \mathrm{sec}$

$7.116 \mathrm{E}+01$

$1.415 \mathrm{E}-04 \mathrm{R} / \mathrm{hr}$ 
HNF-SD-WM-CN-091

Rev. 0

Page 43 of 64

New UNH Tank (H0-64-5473) Line Source - Middle

Photon Production Rate for Each Radionuclide:

\begin{tabular}{|c|c|c|c|c|c|c|}
\hline $\begin{array}{l}\gg>Z R- \\
\text { Group } \\
\text { No. }\end{array}$ & $\begin{array}{l}95 \\
\text { Photon } \\
\text { Mean }\end{array}$ & $\begin{array}{l}Z=40) \\
\text { Energy, } \\
\text { Low }\end{array}$ & $\begin{array}{l}\text { MeV } \\
\mathrm{High}\end{array}$ & $\begin{array}{l}\text { eight (117) } \\
\text { Photon Proo } \\
\text { Gamma\&Xray }\end{array}$ & $\begin{array}{c}\text { 7. } 180 \mathrm{E}+0 \mathrm{l} \\
\text { ction Rate } \\
\text { Bremss. }\end{array}$ & $\begin{array}{c}\text { Total } \\
\text { photon/s }\end{array}$ \\
\hline $\begin{array}{r}1 \\
2 \\
3 \\
4 \\
5 \\
6 \\
7 \\
8 \\
9 \\
10 \\
11 \\
12 \\
13 \\
14 \\
15 \\
16 \\
17\end{array}$ & $\begin{array}{l}0.0145 \\
0.0242 \\
0.0347 \\
0.0446 \\
0.0547 \\
0.0647 \\
0.0748 \\
0.0847 \\
0.0948 \\
0.1288 \\
0.2290 \\
0.3347 \\
0.4551 \\
0.7242 \\
0.7567 \\
0.9332 \\
1.1000\end{array}$ & $\begin{array}{l}0.01 \\
0.02 \\
0.03 \\
0.04 \\
0.05 \\
0.06 \\
0.07 \\
0.08 \\
0.09 \\
0.10 \\
0.20 \\
0.30 \\
0.40 \\
0.55 \\
0.75 \\
0.90 \\
1.10\end{array}$ & $\begin{array}{l}0.02 \\
0.03 \\
0.04 \\
0.05 \\
0.06 \\
0.07 \\
0.08 \\
0.09 \\
0.10 \\
0.20 \\
0.30 \\
0.40 \\
0.55 \\
0.75 \\
0.90 \\
1.10 \\
1.35\end{array}$ & $\begin{array}{l}0.000 \mathrm{E}+00 \\
0.000 \mathrm{E}+00 \\
0.000 \mathrm{E}+00 \\
0.000 \mathrm{E}+00 \\
0.000 \mathrm{E}+00 \\
0.000 \mathrm{E}+00 \\
0.000 \mathrm{E}+00 \\
0.000 \mathrm{E}+00 \\
0.000 \mathrm{E}+00 \\
0.000 \mathrm{E}+00 \\
0.000 \mathrm{E}+00 \\
0.000 \mathrm{E}+00 \\
0.000 \mathrm{E}+00 \\
1.161 \mathrm{E}+06 \\
1.469 \mathrm{E}+06 \\
0.000 \mathrm{E}+00 \\
0.000 \mathrm{E}+00\end{array}$ & $\begin{array}{l}9.895 \mathrm{E}+03 \\
4.617 \mathrm{E}+03 \\
2.128 \mathrm{E}+03 \\
1.340 \mathrm{E}+03 \\
8.960 \mathrm{E}+02 \\
6.168 \mathrm{E}+02 \\
4.481 \mathrm{E}+02 \\
3.298 \mathrm{E}+02 \\
2.485 \mathrm{E}+02 \\
7.761 \mathrm{E}+02 \\
6.182 \mathrm{E}+01 \\
7.091 \mathrm{E}+00 \\
2.510 \mathrm{E}+00 \\
5.022 \mathrm{E}-01 \\
3.012 \mathrm{E}-02 \\
2.489 \mathrm{E}-03 \\
2.519 \mathrm{E}-07\end{array}$ & $\begin{array}{l}9.895 \mathrm{E}+03 \\
4.617 \mathrm{E}+03 \\
2.128 \mathrm{E}+03 \\
1.340 \mathrm{E}+03 \\
8.960 \mathrm{E}+02 \\
6.168 \mathrm{E}+02 \\
4.481 \mathrm{E}+02 \\
3.298 \mathrm{E}+02 \\
2.485 \mathrm{E}+02 \\
7.761 \mathrm{E}+02 \\
6.182 \mathrm{E}+01 \\
7.091 \mathrm{E}+00 \\
2.510 \mathrm{E}+00 \\
1.161 \mathrm{E}+06 \\
1.469 \mathrm{E}+06 \\
2.489 \mathrm{E}-03 \\
2.519 \mathrm{E}-07\end{array}$ \\
\hline
\end{tabular}

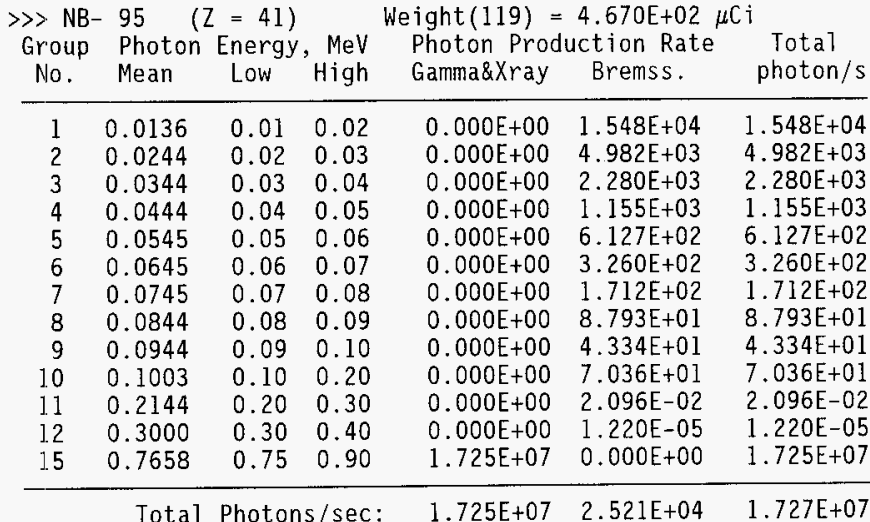

Total Photons/sec: $\quad 1.725 \mathrm{E}+07 \quad 2.521 \mathrm{E}+04 \quad 1.727 \mathrm{E}+07$ 
HNF-SD-WM-CN-091

Rev. 0

Page 44 of 64

\begin{tabular}{|c|c|c|c|c|c|c|}
\hline $\begin{array}{l}>\text { RU } \\
\text { Group } \\
\text { No. }\end{array}$ & $\begin{array}{l}\text { Photon } \\
\text { Mean }\end{array}$ & $\begin{array}{c}Z=44 \text { ) } \\
\text { Energy, } \\
\text { Low }\end{array}$ & $\begin{array}{l}\mathrm{MeV} \\
\mathrm{High}\end{array}$ & $\begin{array}{l}\text { leight (155) } \\
\text { Photon Pro } \\
\text { Gamma\&Xray }\end{array}$ & $\begin{array}{c}3.960 E+01 \\
\text { ction Rate } \\
\text { Bremss. }\end{array}$ & $\begin{array}{l}\text { Total } \\
\text { photon/s }\end{array}$ \\
\hline $\begin{array}{r}1 \\
2 \\
3 \\
4 \\
5 \\
6 \\
7 \\
8 \\
9 \\
10 \\
11 \\
12 \\
13 \\
14\end{array}$ & $\begin{array}{l}0.0137 \\
0.0204 \\
0.0345 \\
0.0445 \\
0.0530 \\
0.0647 \\
0.0747 \\
0.0847 \\
0.0947 \\
0.1293 \\
0.2968 \\
0.3381 \\
0.4978 \\
0.6044\end{array}$ & $\begin{array}{l}0.01 \\
0.02 \\
0.03 \\
0.04 \\
0.05 \\
0.06 \\
0.07 \\
0.08 \\
0.09 \\
0.10 \\
0.20 \\
0.30 \\
0.40 \\
0.55\end{array}$ & $\begin{array}{l}0.02 \\
0.03 \\
0.04 \\
0.05 \\
0.06 \\
0.07 \\
0.08 \\
0.09 \\
0.10 \\
0.20 \\
0.30 \\
0.40 \\
0.55 \\
0.75\end{array}$ & $\begin{array}{l}0.000 \mathrm{E}+00 \\
6.886 \mathrm{E}+03 \\
0.000 \mathrm{E}+00 \\
0.000 \mathrm{E}+00 \\
5.275 \mathrm{E}+03 \\
0.000 \mathrm{E}+00 \\
0.000 \mathrm{E}+00 \\
0.000 \mathrm{E}+00 \\
0.000 \mathrm{E}+00 \\
0.000 \mathrm{E}+00 \\
3.956 \mathrm{E}+03 \\
0.000 \mathrm{E}+00 \\
1.295 \mathrm{E}+06 \\
1.061 \mathrm{E}+05\end{array}$ & $\begin{array}{l}2.000 \mathrm{E}+03 \\
7.766 \mathrm{E}+02 \\
4.206 \mathrm{E}+02 \\
2.412 \mathrm{E}+02 \\
1.370 \mathrm{E}+02 \\
9.047 \mathrm{E}+01 \\
6.118 \mathrm{E}+01 \\
4.071 \mathrm{E}+01 \\
2.861 \mathrm{E}+01 \\
8.818 \mathrm{E}+01 \\
1.428 \mathrm{E}+01 \\
3.394 \mathrm{E}+00 \\
8.038 \mathrm{E}-01 \\
4.370 \mathrm{E}-02\end{array}$ & $\begin{array}{l}2.000 \mathrm{E}+03 \\
7.663 \mathrm{E}+03 \\
4.206 \mathrm{E}+02 \\
2.412 \mathrm{E}+02 \\
5.412 \mathrm{E}+03 \\
9.047 \mathrm{E}+01 \\
6.118 \mathrm{E}+01 \\
4.071 \mathrm{E}+01 \\
2.861 \mathrm{E}+01 \\
8.818 \mathrm{E}+01 \\
3.970 \mathrm{E}+03 \\
3.394 \mathrm{E}+00 \\
1.295 \mathrm{E}+06 \\
1.061 \mathrm{E}+05\end{array}$ \\
\hline
\end{tabular}

\begin{tabular}{|c|c|c|c|c|c|c|}
\hline $\begin{array}{l}\text { \> RU } \\
\text { Group } \\
\text { No. }\end{array}$ & $\begin{array}{l}-106 \\
\text { Photon } \\
\text { Mean }\end{array}$ & $\begin{array}{c}Z=44) \\
\text { Energy, } \\
\text { Low }\end{array}$ & $\begin{array}{r}\text { MeV } \\
\text { High }\end{array}$ & $\begin{array}{l}\text { Weight }(170)= \\
\text { Photon Proc } \\
\text { Gamma\&Xray }\end{array}$ & $\begin{array}{c}5.330 \mathrm{E}+02 \\
\text { luction Rate } \\
\text { Bremss. }\end{array}$ & $\begin{array}{c}\text { Total } \\
\text { photon/s }\end{array}$ \\
\hline $\begin{array}{l}1 \\
2 \\
3\end{array}$ & $\begin{array}{l}0.0129 \\
0.0225 \\
0.0304\end{array}$ & $\begin{array}{l}0.01 \\
0.02 \\
0.03\end{array}$ & $\begin{array}{l}0.02 \\
0.03 \\
0.04\end{array}$ & $\begin{array}{l}0.000 \mathrm{E}+00 \\
0.000 \mathrm{E}+00 \\
0.000 \mathrm{E}+00\end{array}$ & $\begin{array}{l}6.770 E+02 \\
4.482 E+01 \\
1.234 E+00\end{array}$ & $\begin{array}{l}6.770 E+02 \\
4.482 E+01 \\
1.234 E+00\end{array}$ \\
\hline
\end{tabular}

Total Photons/sec: $\quad 0.000 E+00 \quad 7.230 E+02 \quad 7.230 E+02$ 
HNF-SD-WM-CN-091

Rev. 0

Page 45 of 64

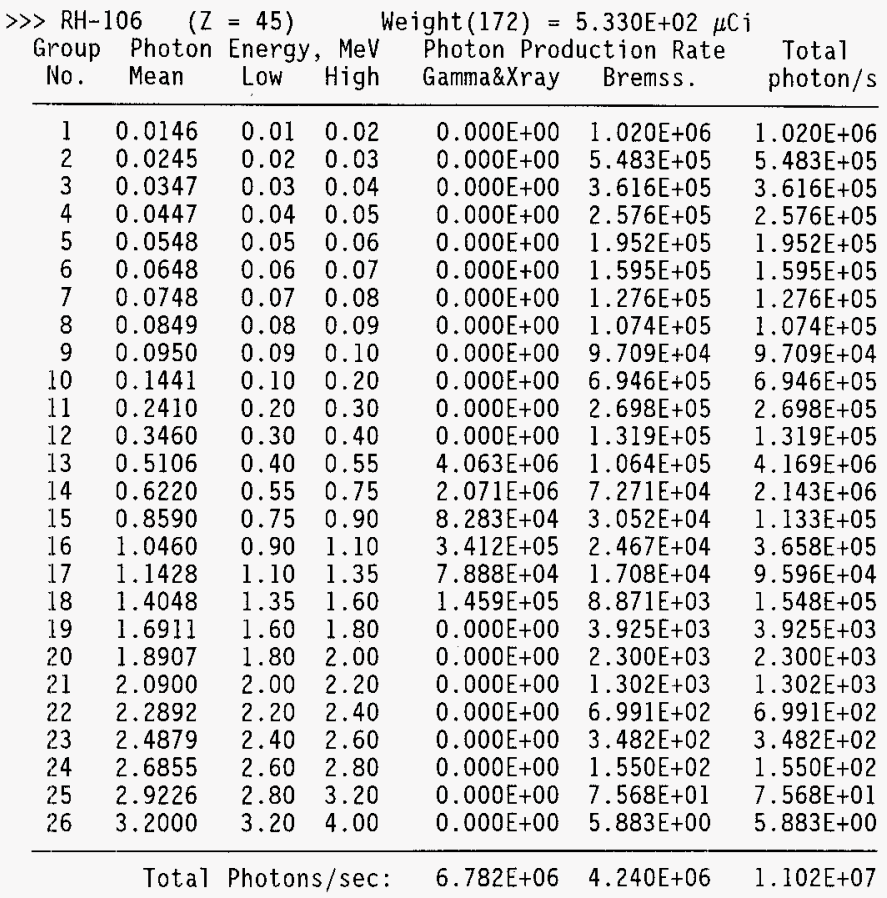


HNF-SD-WM-CN-091

Rev. 0

Page 46 of 64

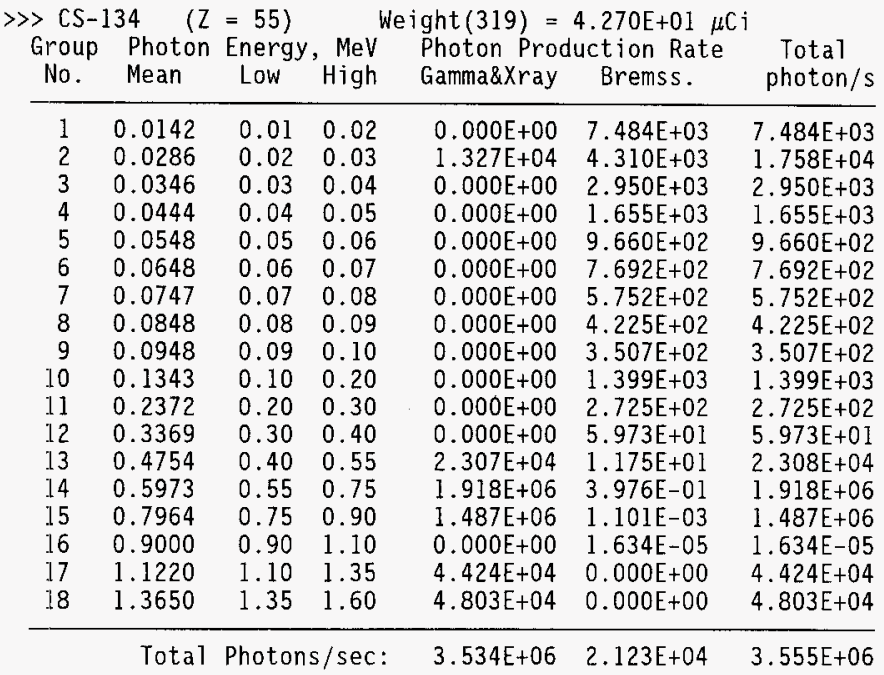

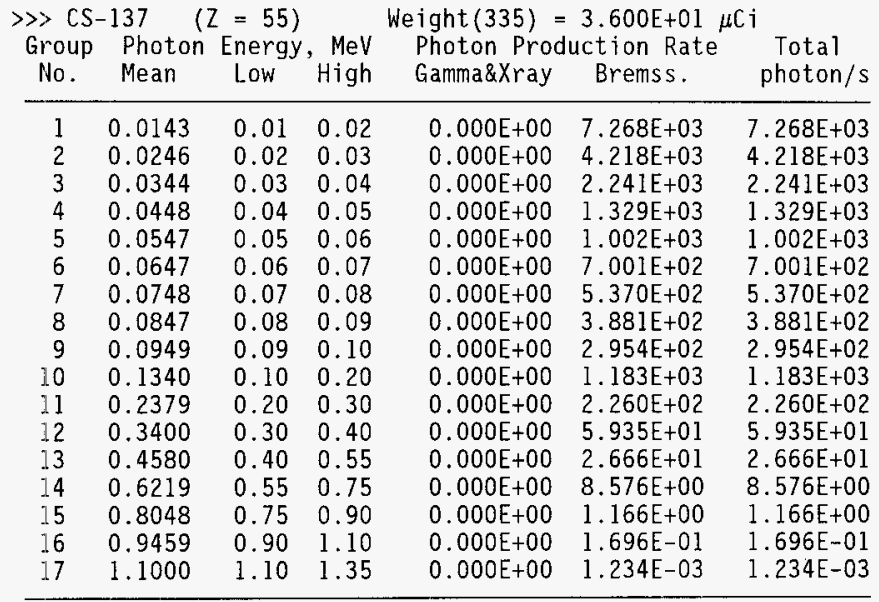

Total Photons/sec: $0.000 \mathrm{E}+00 \quad 1.948 \mathrm{E}+04 \quad 1.948 \mathrm{E}+04$ 
HNF-SD-WM-CN-091

Rev. 0

Page 47 of 64

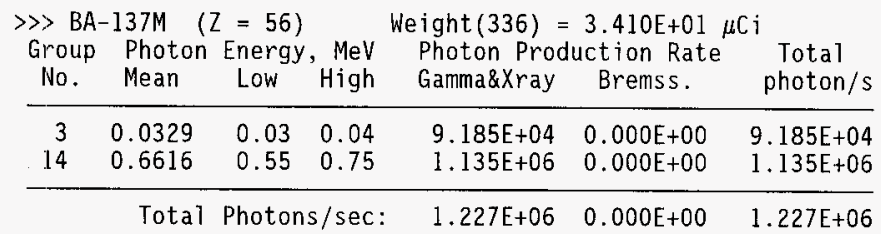

\begin{tabular}{|c|c|c|c|c|c|c|}
\hline$\gg \mathrm{CE}$ & -144 & 58) & & Weight (376) & $5.810 E+02$ & \\
\hline Group & Photon & Energy & $\mathrm{MeV}$ & Photon Pr & uction Rate & Total \\
\hline & & & & Imma\&Xray & emss. & photon/s \\
\hline 1 & 0.0143 & 0.01 & 0.02 & $0.000 \mathrm{E}+00$ & $5.672 E+04$ & $5.672 \mathrm{E}+04$ \\
\hline 2 & 0.0241 & 0.02 & 0.03 & $0.000 \mathrm{E}+00$ & $1.944 E+04$ & $E+04$ \\
\hline 3 & 0.0358 & 0.03 & 0.04 & $1.550 \mathrm{E}+06$ & $1.034 E+04$ & 1.560E+06 \\
\hline 4 & 0.0409 & 0.04 & 0.05 & $4.428 \mathrm{E}+05$ & $6.003 \mathrm{E}+03$ & $88 E+05$ \\
\hline 5 & 0.0546 & 0.05 & 0.06 & $0.000 \mathrm{E}+00$ & $3.815 \mathrm{E}+03$ & $3.815 E+03$ \\
\hline 6 & 0.0647 & 0.06 & 0.07 & $0.000 \mathrm{E}+00$ & $2.609 \mathrm{E}+03$ & $2.609 \mathrm{E}+03$ \\
\hline 7 & 0.0747 & 0.07 & 0.08 & $0.000 \mathrm{E}+00$ & $1.777 \mathrm{E}+03$ & $1.777 \mathrm{E}+03$ \\
\hline 8 & 0.0801 & 0.08 & 0.09 & $3.440 \mathrm{E}+05$ & $1.251 \mathrm{E}+03$ & $3.452 E+05$ \\
\hline 9 & 0.0947 & 0.09 & 0.10 & $0.000 E+00$ & $8.952 \mathrm{E}+02$ & $8.952 \mathrm{E}+02$ \\
\hline 10 & 0.1335 & 0.10 & 0.20 & 2. $322 \mathrm{E}+06$ & $2.278 E+03$ & $2.324 \mathrm{E}+06$ \\
\hline |l 1 & 0.2139 & 0.20 & 0.30 & $0.000 \mathrm{E}+00$ & $5.692 \mathrm{E}+01$ & $5.692 \mathrm{E}+01$ \\
\hline 12 & 0.3000 & 0.30 & 0.40 & $0.000 \mathrm{E}+00$ & $2.197 \mathrm{E}-02$ & $2.197 \mathrm{E}-02$ \\
\hline
\end{tabular}

Total Photons/sec: $4.658 \mathrm{E}+06 \quad 1.052 \mathrm{E}+05 \quad 4.764 \mathrm{E}+06$ 
HNF-SD-WM-CN-091

Rev. 0

Page 48 of 64

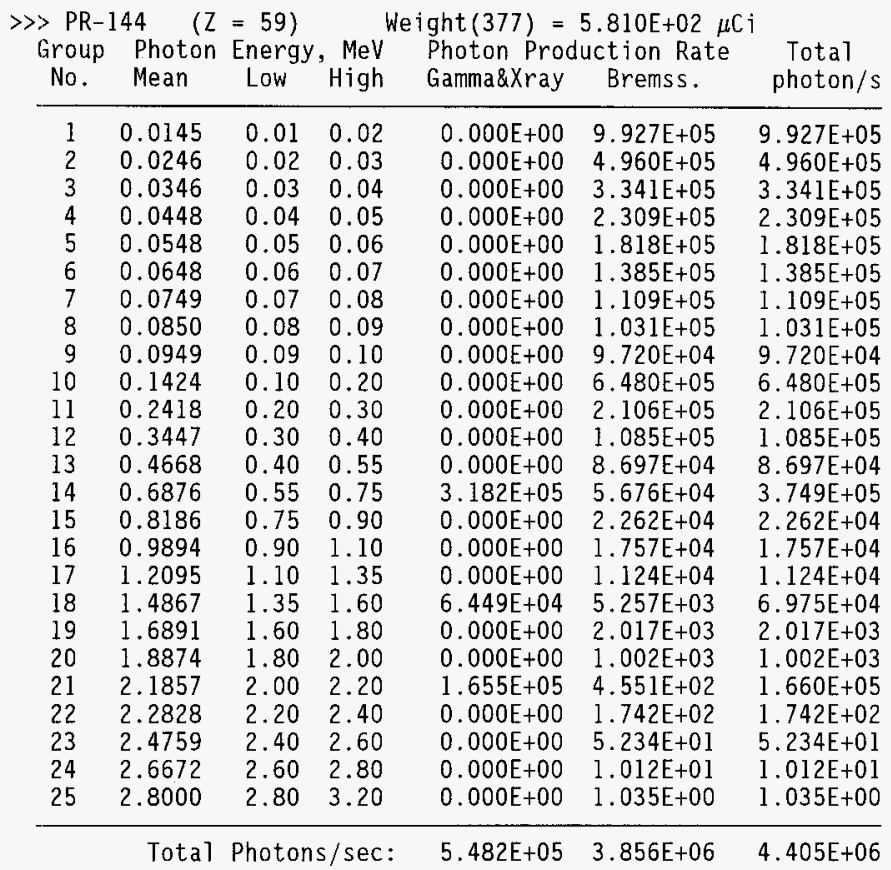

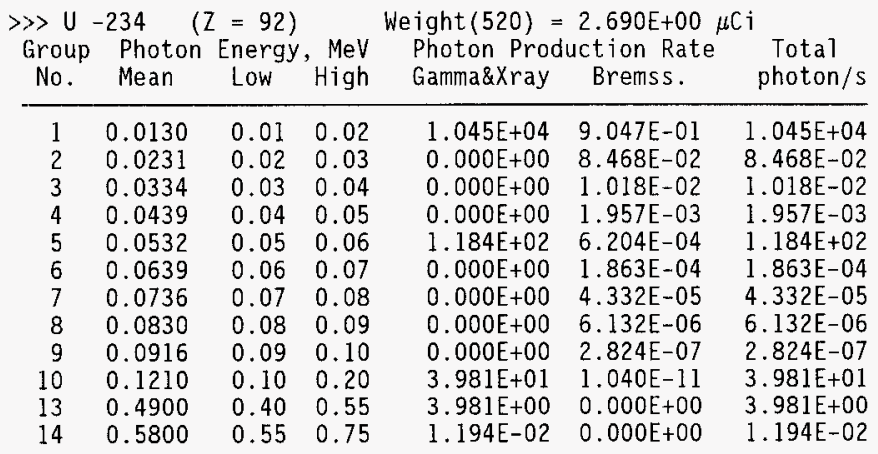

Total Photons/sec: $1.061 \mathrm{E}+04 \quad 1.002 \mathrm{E}+00 \quad 1.061 \mathrm{E}+04$ 
HNF-SD-WM-CN-091

Rev. 0

Page 49 of 64

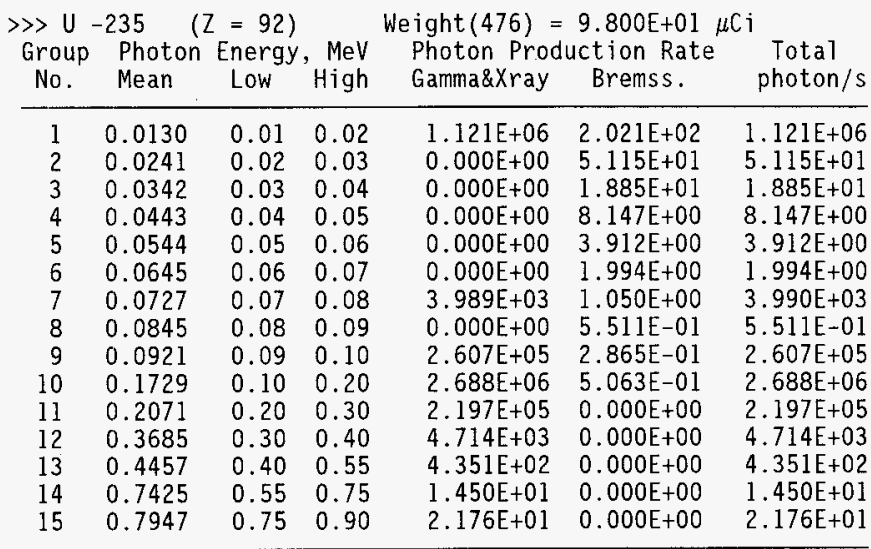

Total Photons/sec: $4.298 \mathrm{E}+06 \quad 2.885 \mathrm{E}+02 \quad 4.298 \mathrm{E}+06$

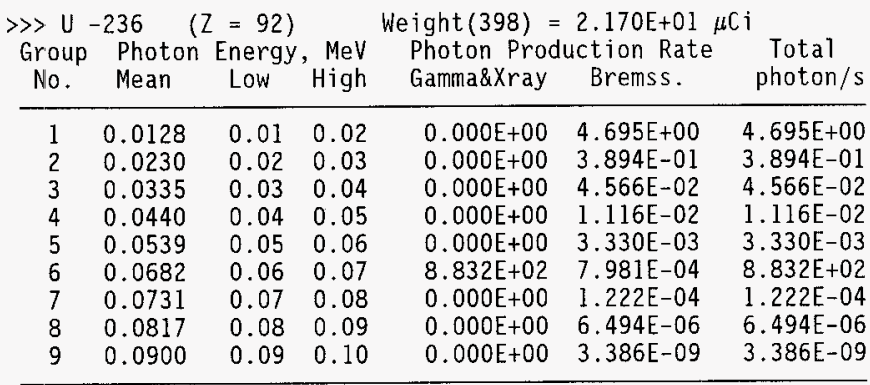

Total Photons/sec: $\quad 8.832 \mathrm{E}+02 \quad 5.146 \mathrm{E}+00 \quad 8.883 \mathrm{E}+02$ 


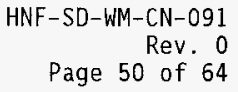

\begin{tabular}{cccccccc}
$\gg>\mathrm{U}-238$ & $(Z=92)$ & \multicolumn{4}{c}{ Weight $(526)=1.590 \mathrm{E}+04 \mu \mathrm{Ci}$} \\
$\begin{array}{c}\text { Group } \\
\text { No. }\end{array}$ & $\begin{array}{c}\text { Photon } \\
\text { Mean }\end{array}$ & $\begin{array}{c}\text { Energy, } \\
\text { Low }\end{array}$ & MeV & $\begin{array}{c}\text { Photon Production Rate } \\
\text { Gigmma\&Xray }\end{array}$ & $\begin{array}{c}\text { Total } \\
\text { Bremss. }\end{array}$ & photon/s \\
\hline 1 & 0.0130 & 0.01 & 0.02 & $5.195 \mathrm{E}+07$ & $3.094 \mathrm{E}+03$ & $5.195 \mathrm{E}+07$ \\
2 & 0.0230 & 0.02 & 0.03 & $0.000 \mathrm{E}+00$ & $2.563 \mathrm{E}+02$ & $2.563 \mathrm{E}+02$ \\
3 & 0.0335 & 0.03 & 0.04 & $0.000 \mathrm{E}+00$ & $2.914 \mathrm{E}+01$ & $2.914 \mathrm{E}+01$ \\
4 & 0.0440 & 0.04 & 0.05 & $0.000 \mathrm{E}+00$ & $6.644 \mathrm{E}+00$ & $6.644 \mathrm{E}+00$ \\
5 & 0.0538 & 0.05 & 0.06 & $0.000 \mathrm{E}+00$ & $1.823 \mathrm{E}+00$ & $1.823 \mathrm{E}+00$ \\
6 & 0.0664 & 0.06 & 0.07 & $5.883 \mathrm{E}+05$ & $3.766 \mathrm{E}-01$ & $5.883 \mathrm{E}+05$ \\
7 & 0.0729 & 0.07 & 0.08 & $0.000 \mathrm{E}+00$ & $4.775 \mathrm{E}-02$ & $4.775 \mathrm{E}-02$ \\
8 & 0.0808 & 0.08 & 0.09 & $0.000 \mathrm{E}+00$ & $2.309 \mathrm{E}-03$ & $2.309 \mathrm{E}-03$ \\
\hline
\end{tabular}


HNF-SD-WM-CN-091

Rev. 0

Page 51 of 64

New UNH Tank (H0-64-5473) Line Source - Middle

Shield Composition, $g / c c$

Shield 1 Shield 2 Shield 3

\begin{tabular}{llll}
\hline AIR & $0.000 \mathrm{E}+00$ & $0.000 \mathrm{E}+00$ & $1.290 \mathrm{E}-03$ \\
ALUMINUM & $1.665 \mathrm{E}+00$ & $0.000 \mathrm{E}+00$ & $0.000 \mathrm{E}+00$ \\
IRON & $0.000 \mathrm{E}+00$ & $7.800 \mathrm{E}+00$ & $0.000 \mathrm{E}+00$
\end{tabular}

Totals: $\quad 1.665 \mathrm{E}+00 \quad 7.800 \mathrm{E}+00 \quad 1.290 \mathrm{E}-03$

$E$, MeV Linear Attenuation, per $\mathrm{cm}$ (last region is air)

$\begin{array}{llll}0.0131 & 2.004 \mathrm{E}+01 & 6.779 \mathrm{E}+02 & 2.838 \mathrm{E}-03 \\ 0.0245 & 3.229 \mathrm{E}+00 & 1.146 \mathrm{E}+02 & 6.409 \mathrm{E}-04 \\ 0.0354 & 1.236 \mathrm{E}+00 & 4.037 \mathrm{E}+01 & 3.627 \mathrm{E}-04 \\ 0.0429 & 8.096 \mathrm{E}-01 & 2.318 \mathrm{E}+01 & 2.979 \mathrm{E}-04 \\ 0.0548 & 5.145 \mathrm{E}-01 & 1.182 \mathrm{E}+01 & 2.497 \mathrm{E}-04 \\ 0.0659 & 4.008 \mathrm{E}-01 & 7.416 \mathrm{E}+00 & 2.302 \mathrm{E}-04 \\ 0.0748 & 3.516 \mathrm{E}-01 & 5.442 \mathrm{E}+00 & 2.186 \mathrm{E}-04 \\ 0.0819 & 3.254 \mathrm{E}-01 & 4.420 \mathrm{E}+00 & 2.117 \mathrm{E}-04 \\ 0.0933 & 2.947 \mathrm{E}-01 & 3.335 \mathrm{E}+00 & 2.030 \mathrm{E}-04 \\ 0.1522 & 2.284 \mathrm{E}-01 & 1.508 \mathrm{E}+00 & 1.749 \mathrm{E}-04 \\ 0.2310 & 1.920 \mathrm{E}-01 & 1.031 \mathrm{E}+00 & 1.518 \mathrm{E}-04 \\ 0.3458 & 1.636 \mathrm{E}-01 & 7.918 \mathrm{E}-01 & 1.304 \mathrm{E}-04 \\ 0.5068 & 1.399 \mathrm{E}-01 & 6.509 \mathrm{E}-01 & 1.114 \mathrm{E}-04 \\ 0.6423 & 1.259 \mathrm{E}-01 & 5.802 \mathrm{E}-01 & 1.006 \mathrm{E}-04 \\ 0.7680 & 1.160 \mathrm{E}-01 & 5.316 \mathrm{E}-01 & 9.281 \mathrm{E}-05 \\ 1.0434 & 1.001 \mathrm{E}-01 & 4.565 \mathrm{E}-01 & 8.021 \mathrm{E}-05 \\ 1.1417 & 9.565 \mathrm{E}-02 & 4.360 \mathrm{E}-01 & 7.665 \mathrm{E}-05 \\ 1.4187 & 8.565 \mathrm{E}-02 & 3.906 \mathrm{E}-01 & 6.855 \mathrm{E}-05 \\ 1.6904 & 7.831 \mathrm{E}-02 & 3.585 \mathrm{E}-01 & 6.267 \mathrm{E}-05 \\ 1.8897 & 7.401 \mathrm{E}-02 & 3.402 \mathrm{E}-01 & 5.906 \mathrm{E}-05 \\ 2.1850 & 6.883 \mathrm{E}-02 & 3.188 \mathrm{E}-01 & 5.431 \mathrm{E}-05 \\ 2.2879 & 6.728 \mathrm{E}-02 & 3.124 \mathrm{E}-01 & 5.280 \mathrm{E}-05 \\ 2.4864 & 6.458 \mathrm{E}-02 & 3.017 \mathrm{E}-01 & 5.019 \mathrm{E}-05 \\ 2.6844 & 6.221 \mathrm{E}-02 & 2.926 \mathrm{E}-01 & 4.795 \mathrm{E}-05 \\ 2.9209 & 5.971 \mathrm{E}-02 & 2.836 \mathrm{E}-01 & 4.577 \mathrm{E}-05 \\ 3.2000 & 5.717 \mathrm{E}-02 & 2.751 \mathrm{E}-01 & 4.383 \mathrm{E}-05\end{array}$


HNF-SD-WM-CN-091

Rev. 0

Page 52 of 64

New UNH Tank (H0-64-5473) Line Source - Middle

Source Shields Distance to Detector, $X=2.000 \mathrm{E}+00 \mathrm{~cm}$

Cylindrical Cylindrical Source Volume $=2.356 \mathrm{E}+03 \mathrm{cc}$

Source Mass $=3.923 \mathrm{E}+03$ grams

Source Length $=7.500 E+02 \mathrm{~cm} \quad$ Distance Along $C y l$ inder, $Y=3.750 E+02 \mathrm{~cm}$

Integration Specs: $\quad$ NTHETA $=11 \quad$ NPSI $=11 \quad$ DELR computed internally

Total Intervals: $1.038 E+05$ (photon source is the lst region)

Shield Thickness: $1.000 \mathrm{E}+00,6.350 \mathrm{E}-01,3.650 \mathrm{E}-01 \mathrm{~cm}$

Distances from Dose Point to the Outside of

(1) Source Region: $1.000 \mathrm{E}+00 \mathrm{~cm}$ (2) Next Layer: $3.650 \mathrm{E}-01 \mathrm{~cm}$

Dose Buildup Data for Shield 2 with Effective Atomic Number 26.00

Buildup Material is Iron

Source values are interpreted as $\mu \mathrm{C} i$

Source Scale Factor was $1.000 E+03$

Fluence-to-Dose Conversion: Photons in Air

Average Source Total Fluence to Energy Fluence Dose Rate

E, MeV photons $/ \mathrm{sec}$ Dose Factor $\mathrm{MeV} / \mathrm{cm}^{2} / \mathrm{s} \mathrm{R} / \mathrm{hr}$

\begin{tabular}{|c|c|c|c|c|}
\hline $\begin{array}{l}0.0131 \\
0.0245 \\
0.0354 \\
0.0429 \\
0.0548 \\
0.0659 \\
0.0748 \\
0.0819 \\
0.0933 \\
0.1522 \\
0.2310 \\
0.3458 \\
0.5068 \\
0.6423 \\
0.7680 \\
1.0434 \\
1.1417 \\
1.4187 \\
1.6904 \\
1.8897 \\
2.1850 \\
2.2879 \\
2.4864 \\
2.6844 \\
2.9209 \\
3.2000\end{array}$ & $\begin{array}{l}5.519 \mathrm{E}+07 \\
1.103 \mathrm{E}+06 \\
2.358 \mathrm{E}+06 \\
9.431 \mathrm{E}+05 \\
3.898 \mathrm{E}+05 \\
8.923 \mathrm{E}+05 \\
2.460 \mathrm{E}+05 \\
5.570 \mathrm{E}+05 \\
4.569 \mathrm{E}+05 \\
6.358 \mathrm{E}+06 \\
7.047 \mathrm{E}+05 \\
2.452 \mathrm{E}+05 \\
5.574 \mathrm{E}+06 \\
6.839 \mathrm{E}+06 \\
2.034 \mathrm{E}+07 \\
3.834 \mathrm{E}+05 \\
1.514 \mathrm{E}+05 \\
2.726 \mathrm{E}+05 \\
5.942 \mathrm{E}+03 \\
3.301 \mathrm{E}+03 \\
1.673 \mathrm{E}+05 \\
8.734 \mathrm{E}+02 \\
4.005 \mathrm{E}+02 \\
1.651 \mathrm{E}+02 \\
7.672 \mathrm{E}+01 \\
5.883 \mathrm{E}+00\end{array}$ & $\begin{array}{l}1.263 \mathrm{E}-04 \\
1.754 \mathrm{E}-05 \\
5.931 \mathrm{E}-06 \\
3.621 \mathrm{E}-06 \\
2.232 \mathrm{E}-06 \\
1.811 \mathrm{E}-06 \\
1.667 \mathrm{E}-06 \\
1.621 \mathrm{E}-06 \\
1.594 \mathrm{E}-06 \\
1.734 \mathrm{E}-06 \\
1.901 \mathrm{E}-06 \\
2.016 \mathrm{E}-06 \\
2.040 \mathrm{E}-06 \\
2.030 \mathrm{E}-06 \\
1.997 \mathrm{E}-06 \\
1.907 \mathrm{E}-06 \\
1.875 \mathrm{E}-06 \\
1.790 \mathrm{E}-06 \\
1.714 \mathrm{E}-06 \\
1.663 \mathrm{E}-06 \\
1.604 \mathrm{E}-06 \\
1.593 \mathrm{E}-06 \\
1.538 \mathrm{E}-06 \\
1.502 \mathrm{E}-06 \\
1.465 \mathrm{E}-06 \\
1.425 \mathrm{E}-06\end{array}$ & $\begin{array}{l}0.000 \mathrm{E}+00 \\
0.000 \mathrm{E}+00 \\
3.240 \mathrm{E}-12 \\
1.912 \mathrm{E}-07 \\
3.275 \mathrm{E}-04 \\
2.467 \mathrm{E}-02 \\
3.529 \mathrm{E}-02 \\
1.950 \mathrm{E}-01 \\
4.347 \mathrm{E}-01 \\
7.103 \mathrm{E}+01 \\
1.570 \mathrm{E}+01 \\
9.371 \mathrm{E}+00 \\
3.370 \mathrm{E}+02 \\
5.431 \mathrm{E}+02 \\
1.972 \mathrm{E}+03 \\
5.174 \mathrm{E}+01 \\
2.245 \mathrm{E}+01 \\
5.050 \mathrm{E}+01 \\
1.311 \mathrm{E}+00 \\
8.139 \mathrm{E}-01 \\
4.770 \mathrm{E}+01 \\
2.608 \mathrm{E}-01 \\
1.298 \mathrm{E}-01 \\
5.760 \mathrm{E}-02 \\
2.900 \mathrm{E}-02 \\
2.426 \mathrm{E}-03\end{array}$ & $\begin{array}{l}0.000 \mathrm{E}+00 \\
0.000 \mathrm{E}+00 \\
1.922 \mathrm{E}-17 \\
6.923 \mathrm{E}-13 \\
7.310 \mathrm{E}-10 \\
4.469 \mathrm{E}-08 \\
5.881 \mathrm{E}-08 \\
3.161 \mathrm{E}-07 \\
6.928 \mathrm{E}-07 \\
1.231 \mathrm{E}-04 \\
2.986 \mathrm{E}-05 \\
1.889 \mathrm{E}-05 \\
6.875 \mathrm{E}-04 \\
1.102 \mathrm{E}-03 \\
3.937 \mathrm{E}-03 \\
9.870 \mathrm{E}-05 \\
4.210 \mathrm{E}-05 \\
9.042 \mathrm{E}-05 \\
2.248 \mathrm{E}-06 \\
1.353 \mathrm{E}-06 \\
7.650 \mathrm{E}-05 \\
4.154 \mathrm{E}-07 \\
1.996 \mathrm{E}-07 \\
8.650 \mathrm{E}-08 \\
4.247 \mathrm{E}-08 \\
3.457 \mathrm{E}-09\end{array}$ \\
\hline Totals: & $1.032 \mathrm{E}+08$ & ons/sec & $3.124 \mathrm{E}+03$ & $6.212 \mathrm{E}-03$ \\
\hline
\end{tabular}


HNF-SD-WM-CN-091

Rev. 0

Page 53 of 64

New UNH Tank (H0-64-5473) Annular Source - Middle

Shield Composition, $g / c c$

Shield 1 Shield 2 Shield 3 Shield 4

\begin{tabular}{lllll}
\hline AIR & $1.290 \mathrm{E}-03$ & $0.000 \mathrm{E}+00$ & $0.000 \mathrm{E}+00$ & $1.290 \mathrm{E}-03$ \\
ALUMINUM & $0.000 \mathrm{E}+00$ & $1.665 \mathrm{E}+00$ & $0.000 \mathrm{E}+00$ & $0.000 \mathrm{E}+00$ \\
IRON & $0.000 \mathrm{E}+00$ & $0.000 \mathrm{E}+00$ & $7.800 \mathrm{E}+00$ & $0.000 \mathrm{E}+00$ \\
\hline Totals: & $1.290 \mathrm{E}-03$ & $1.665 \mathrm{E}+00$ & $7.800 \mathrm{E}+00$ & $1.290 \mathrm{E}-03$ \\
\hline
\end{tabular}

E, MeV Linear Attenuation, per $\mathrm{cm}$ (1ast region is air)

$\begin{array}{lllll}0.0131 & 2.838 \mathrm{E}-03 & 2.004 \mathrm{E}+01 & 6.779 \mathrm{E}+02 & 2.838 \mathrm{E}-03 \\ 0.0245 & 6.409 \mathrm{E}-04 & 3.229 \mathrm{E}+00 & 1.146 \mathrm{E}+02 & 6.409 \mathrm{E}-04 \\ 0.0354 & 3.627 \mathrm{E}-04 & 1.236 \mathrm{E}+00 & 4.037 \mathrm{E}+01 & 3.627 \mathrm{E}-04 \\ 0.0429 & 2.979 \mathrm{E}-04 & 8.096 \mathrm{E}-01 & 2.318 \mathrm{E}+01 & 2.979 \mathrm{E}-04 \\ 0.0548 & 2.497 \mathrm{E}-04 & 5.145 \mathrm{E}-01 & 1.182 \mathrm{E}+01 & 2.497 \mathrm{E}-04 \\ 0.0659 & 2.302 \mathrm{E}-04 & 4.008 \mathrm{E}-01 & 7.416 \mathrm{E}+00 & 2.302 \mathrm{E}-04 \\ 0.0748 & 2.186 \mathrm{E}-04 & 3.516 \mathrm{E}-01 & 5.442 \mathrm{E}+00 & 2.186 \mathrm{E}-04 \\ 0.0819 & 2.117 \mathrm{E}-04 & 3.254 \mathrm{E}-01 & 4.420 \mathrm{E}+00 & 2.117 \mathrm{E}-04 \\ 0.0933 & 2.030 \mathrm{E}-04 & 2.947 \mathrm{E}-01 & 3.335 \mathrm{E}+00 & 2.030 \mathrm{E}-04 \\ 0.1522 & 1.749 \mathrm{E}-04 & 2.284 \mathrm{E}-01 & 1.508 \mathrm{E}+00 & 1.749 \mathrm{E}-04 \\ 0.2310 & 1.518 \mathrm{E}-04 & 1.920 \mathrm{E}-01 & 1.031 \mathrm{E}+00 & 1.518 \mathrm{E}-04 \\ 0.3458 & 1.304 \mathrm{E}-04 & 1.636 \mathrm{E}-01 & 7.918 \mathrm{E}-01 & 1.304 \mathrm{E}-04 \\ 0.5068 & 1.114 \mathrm{E}-04 & 1.399 \mathrm{E}-01 & 6.509 \mathrm{E}-01 & 1.114 \mathrm{E}-04 \\ 0.6423 & 1.006 \mathrm{E}-04 & 1.259 \mathrm{E}-01 & 5.802 \mathrm{E}-01 & 1.006 \mathrm{E}-04 \\ 0.7680 & 9.281 \mathrm{E}-05 & 1.160 \mathrm{E}-01 & 5.316 \mathrm{E}-01 & 9.281 \mathrm{E}-05 \\ 1.0434 & 8.021 \mathrm{E}-05 & 1.001 \mathrm{E}-01 & 4.565 \mathrm{E}-01 & 8.021 \mathrm{E}-05 \\ 1.1417 & 7.665 \mathrm{E}-05 & 9.565 \mathrm{E}-02 & 4.360 \mathrm{E}-01 & 7.665 \mathrm{E}-05 \\ 1.4187 & 6.855 \mathrm{E}-05 & 8.565 \mathrm{E}-02 & 3.906 \mathrm{E}-01 & 6.855 \mathrm{E}-05 \\ 1.6904 & 6.267 \mathrm{E}-05 & 7.831 \mathrm{E}-02 & 3.585 \mathrm{E}-01 & 6.267 \mathrm{E}-05 \\ 1.8897 & 5.906 \mathrm{E}-05 & 7.401 \mathrm{E}-02 & 3.402 \mathrm{E}-01 & 5.906 \mathrm{E}-05 \\ 2.1850 & 5.431 \mathrm{E}-05 & 6.883 \mathrm{E}-02 & 3.188 \mathrm{E}-01 & 5.431 \mathrm{E}-05 \\ 2.2879 & 5.280 \mathrm{E}-05 & 6.728 \mathrm{E}-02 & 3.124 \mathrm{E}-01 & 5.280 \mathrm{E}-05 \\ 2.4864 & 5.019 \mathrm{E}-05 & 6.458 \mathrm{E}-02 & 3.017 \mathrm{E}-01 & 5.019 \mathrm{E}-05 \\ 2.6844 & 4.795 \mathrm{E}-05 & 6.221 \mathrm{E}-02 & 2.926 \mathrm{E}-01 & 4.795 \mathrm{E}-05 \\ 2.9209 & 4.577 \mathrm{E}-05 & 5.971 \mathrm{E}-02 & 2.836 \mathrm{E}-01 & 4.577 \mathrm{E}-05 \\ 3.2000 & 4.383 \mathrm{E}-05 & 5.717 \mathrm{E}-02 & 2.751 \mathrm{E}-01 & 4.383 \mathrm{E}-05\end{array}$


HNF-SD-WM-CN-091

Rev. 0

Page 54 of 64

New UNH Tank (H0-54-5473) Annular Source - Middle

Source Shields Distance to Detector, $X=7.350 E+01 \mathrm{~cm}$

Annular $13 \quad C y 1$. \& Slab Source Volume $=3.414 \mathrm{E}+04 \mathrm{cc}$

Source Mass $=5.684 \mathrm{E}+04$ grams

Source Length $=7.500 E+02 \mathrm{~cm} \quad$ Distance Along Cylinder, $Y=3.750 E+02 \mathrm{~cm}$

Integration Specs: $\quad$ NTHETA $=11$ NPSI $=11$ DELR computed internally

Total Interva7s: $1.861 \mathrm{E}+02$ (photon source is the 2 nd region)

Shield Thickness: $7.240 \mathrm{E}+01,1.000 \mathrm{E}-01,6.350 \mathrm{E}-01,3.650 \mathrm{E}-01 \mathrm{~cm}$

Distances from Dose Point to the Outside of
(1) Source Region: $1.000 \mathrm{E}+00 \mathrm{~cm}$
(2) Next Layer: $3.650 \mathrm{E}-01 \mathrm{~cm}$

Dose Buildup Data for Shield 3 with Effective Atomic Number 26.00

Buildup Material is Iron

Source values are interpreted as $\mu \mathrm{C} \mathbf{i}$

Source Scale Factor was $1.000 E+03$

Fluence-to-Dose Conversion: Photons in Air

\begin{tabular}{ccccc}
$\begin{array}{c}\text { Average } \\
\text { E, MeV }\end{array}$ & $\begin{array}{c}\text { Source Total } \\
\text { photons/sec }\end{array}$ & $\begin{array}{c}\text { Fluence to } \\
\text { Dose Factor }\end{array}$ & $\begin{array}{c}\text { Energy F1uence } \\
\text { MeV/cm²/s }\end{array}$ & $\begin{array}{c}\text { Dose Rate } \\
\text { R/hr }\end{array}$ \\
\hline 0.0131 & $5.519 \mathrm{E}+07$ & $1.263 \mathrm{E}-04$ & $0.000 \mathrm{E}+00$ & $0.000 \mathrm{E}+00$ \\
0.0245 & $1.103 \mathrm{E}+06$ & $1.754 \mathrm{E}-05$ & $0.000 \mathrm{E}+00$ & $0.000 \mathrm{E}+00$ \\
0.0354 & $2.358 \mathrm{E}+05$ & $5.931 \mathrm{E}-06$ & $8.454 \mathrm{E}-14$ & $5.014 \mathrm{E}-19$ \\
0.0429 & $9.431 \mathrm{E}+05$ & $3.621 \mathrm{E}-06$ & $4.054 \mathrm{E}-09$ & $1.468 \mathrm{E}-14$ \\
0.0548 & $3.898 \mathrm{E}+05$ & $2.232 \mathrm{E}-06$ & $6.122 \mathrm{E}-06$ & $1.366 \mathrm{E}-11$ \\
0.0659 & $8.923 \mathrm{E}+05$ & $1.811 \mathrm{E}-06$ & $4.579 \mathrm{E}-04$ & $8.294 \mathrm{E}-10$ \\
0.0748 & $2.460 \mathrm{E}+05$ & $1.667 \mathrm{E}-06$ & $6.732 \mathrm{E}-04$ & $1.122 \mathrm{E}-09$ \\
0.0819 & $5.570 \mathrm{E}+05$ & $1.621 \mathrm{E}-06$ & $3.834 \mathrm{E}-03$ & $6.213 \mathrm{E}-09$ \\
0.0933 & $4.569 \mathrm{E}+05$ & $1.594 \mathrm{E}-06$ & $9.006 \mathrm{E}-03$ & $1.435 \mathrm{E}-08$ \\
0.1522 & $6.358 \mathrm{E}+06$ & $1.734 \mathrm{E}-06$ & $1.847 \mathrm{E}+00$ & $3.202 \mathrm{E}-06$ \\
0.2310 & $7.047 \mathrm{E}+05$ & $1.901 \mathrm{E}-06$ & $4.514 \mathrm{E}-01$ & $8.583 \mathrm{E}-07$ \\
0.3458 & $2.452 \mathrm{E}+05$ & $2.016 \mathrm{E}-06$ & $2.864 \mathrm{E}-01$ & $5.773 \mathrm{E}-07$ \\
0.5068 & $5.574 \mathrm{E}+06$ & $2.040 \mathrm{E}-06$ & $1.071 \mathrm{E}+01$ & $2.185 \mathrm{E}-05$ \\
0.6423 & $6.839 \mathrm{E}+06$ & $2.030 \mathrm{E}-06$ & $1.763 \mathrm{E}+01$ & $3.577 \mathrm{E}-05$ \\
0.7680 & $2.034 \mathrm{E}+07$ & $1.997 \mathrm{E}-06$ & $6.495 \mathrm{E}+01$ & $1.297 \mathrm{E}-04$ \\
1.0434 & $3.834 \mathrm{E}+05$ & $1.907 \mathrm{E}-06$ & $1.744 \mathrm{E}+00$ & $3.327 \mathrm{E}-06$ \\
1.1417 & $1.514 \mathrm{E}+05$ & $1.875 \mathrm{E}-06$ & $7.615 \mathrm{E}-01$ & $1.428 \mathrm{E}-06$ \\
1.4187 & $2.726 \mathrm{E}+05$ & $1.790 \mathrm{E}-06$ & $1.736 \mathrm{E}+00$ & $3.107 \mathrm{E}-06$ \\
1.6904 & $5.942 \mathrm{E}+03$ & $1.714 \mathrm{E}-06$ & $4.546 \mathrm{E}-02$ & $7.794 \mathrm{E}-08$ \\
1.8897 & $3.301 \mathrm{E}+03$ & $1.663 \mathrm{E}-06$ & $2.835 \mathrm{E}-02$ & $4.713 \mathrm{E}-08$ \\
2.1850 & $1.673 \mathrm{E}+05$ & $1.604 \mathrm{E}-06$ & $1.671 \mathrm{E}+00$ & $2.679 \mathrm{E}-06$ \\
2.2879 & $8.734 \mathrm{E}+02$ & $1.593 \mathrm{E}-06$ & $9.148 \mathrm{E}-03$ & $1.457 \mathrm{E}-08$ \\
2.4864 & $4.005 \mathrm{E}+02$ & $1.538 \mathrm{E}-06$ & $4.565 \mathrm{E}-03$ & $7.020 \mathrm{E}-09$ \\
2.6844 & $1.651 \mathrm{E}+02$ & $1.502 \mathrm{E}-06$ & $2.029 \mathrm{E}-03$ & $3.046 \mathrm{E}-09$ \\
2.9209 & $7.672 \mathrm{E}+01$ & $1.465 \mathrm{E}-06$ & $1.022 \mathrm{E}-03$ & $1.497 \mathrm{E}-09$ \\
3.2000 & $5.883 \mathrm{E}+00$ & $1.425 \mathrm{E}-06$ & $8.556 \mathrm{E}-05$ & $1.219 \mathrm{E}-10$ \\
\hline Tota1s: & $1.032 \mathrm{E}+08$ & $\mathrm{photons} / \mathrm{sec}$ & $1.019 \mathrm{E}+02$ & $2.027 \mathrm{E}-04 \mathrm{R} / \mathrm{hr}$
\end{tabular}


New UNH Tank (H0-64-5473) Line Source - End

Shield Composition, g/cc

Shield 1 Shield 2 Shield 3

\begin{tabular}{llll}
\hline AIR & $0.000 \mathrm{E}+00$ & $0.000 \mathrm{E}+00$ & $1.290 \mathrm{E}-03$ \\
ALUMINUM & $1.665 \mathrm{E}+00$ & $0.000 \mathrm{E}+00$ & $0.000 \mathrm{E}+00$ \\
IRON & $0.000 \mathrm{E}+00$ & $7.800 \mathrm{E}+00$ & $0.000 \mathrm{E}+00$ \\
\hline Totals: & $1.665 \mathrm{E}+00$ & $7.800 \mathrm{E}+00$ & $1.290 \mathrm{E}-03$ \\
\hline
\end{tabular}

$\mathrm{E}$, MeV Linear Attenuation, per $\mathrm{cm}$ (last region is air)

\begin{tabular}{llll}
\hline 0.0131 & $2.004 \mathrm{E}+01$ & $6.779 \mathrm{E}+02$ & $2.838 \mathrm{E}-03$ \\
0.0245 & $3.229 \mathrm{E}+00$ & $1.146 \mathrm{E}+02$ & $6.409 \mathrm{E}-04$ \\
0.0354 & $1.236 \mathrm{E}+00$ & $4.037 \mathrm{E}+01$ & $3.627 \mathrm{E}-04$ \\
0.0429 & $8.096 \mathrm{E}-01$ & $2.318 \mathrm{E}+01$ & $2.979 \mathrm{E}-04$ \\
0.0548 & $5.145 \mathrm{E}-01$ & $1.182 \mathrm{E}+01$ & $2.497 \mathrm{E}-04$ \\
0.0659 & $4.008 \mathrm{E}-01$ & $7.416 \mathrm{E}+00$ & $2.302 \mathrm{E}-04$ \\
0.0748 & $3.516 \mathrm{E}-01$ & $5.442 \mathrm{E}+00$ & $2.186 \mathrm{E}-04$ \\
0.0819 & $3.254 \mathrm{E}-01$ & $4.420 \mathrm{E}+00$ & $2.117 \mathrm{E}-04$ \\
0.0933 & $2.947 \mathrm{E}-01$ & $3.335 \mathrm{E}+00$ & $2.030 \mathrm{E}-04$ \\
0.1522 & $2.284 \mathrm{E}-01$ & $1.508 \mathrm{E}+00$ & $1.749 \mathrm{E}-04$ \\
0.2310 & $1.920 \mathrm{E}-01$ & $1.031 \mathrm{E}+00$ & $1.518 \mathrm{E}-04$ \\
0.3458 & $1.636 \mathrm{E}-01$ & $7.918 \mathrm{E}-01$ & $1.304 \mathrm{E}-04$ \\
0.5068 & $1.399 \mathrm{E}-01$ & $6.509 \mathrm{E}-01$ & $1.114 \mathrm{E}-04$ \\
0.6423 & $1.259 \mathrm{E}-01$ & $5.802 \mathrm{E}-01$ & $1.006 \mathrm{E}-04$ \\
0.7680 & $1.160 \mathrm{E}-01$ & $5.316 \mathrm{E}-01$ & $9.281 \mathrm{E}-05$ \\
1.0434 & $1.001 \mathrm{E}-01$ & $4.565 \mathrm{E}-01$ & $8.021 \mathrm{E}-05$ \\
1.1417 & $9.565 \mathrm{E}-02$ & $4.360 \mathrm{E}-01$ & $7.665 \mathrm{E}-05$ \\
1.4187 & $8.565 \mathrm{E}-02$ & $3.906 \mathrm{E}-01$ & $6.855 \mathrm{E}-05$ \\
1.6904 & $7.831 \mathrm{E}-02$ & $3.585 \mathrm{E}-01$ & $6.267 \mathrm{E}-05$ \\
1.8897 & $7.401 \mathrm{E}-02$ & $3.402 \mathrm{E}-01$ & $5.906 \mathrm{E}-05$ \\
2.1850 & $6.883 \mathrm{E}-02$ & $3.188 \mathrm{E}-01$ & $5.431 \mathrm{E}-05$ \\
2.2879 & $6.728 \mathrm{E}-02$ & $3.124 \mathrm{E}-01$ & $5.280 \mathrm{E}-05$ \\
2.4864 & $6.458 \mathrm{E}-02$ & $3.017 \mathrm{E}-01$ & $5.019 \mathrm{E}-05$ \\
2.6844 & $6.221 \mathrm{E}-02$ & $2.926 \mathrm{E}-01$ & $4.795 \mathrm{E}-05$ \\
2.9209 & $5.971 \mathrm{E}-02$ & $2.836 \mathrm{E}-01$ & $4.577 \mathrm{E}-05$ \\
3.2000 & $5.717 \mathrm{E}-02$ & $2.751 \mathrm{E}-01$ & $4.383 \mathrm{E}-05$
\end{tabular}


HNF-SD-WM-CN-091

Rev. 0

Page 56 of 64

New UNH Tank (H0-64-5473) Line Source - End

Source Shields Distance to Detector, $X=2.000 E+00 \mathrm{~cm}$

Cylindrical Cylindrical Source Volume $=2.356 \mathrm{E}+03 \mathrm{cC}$

Source Mass $=3.923 \mathrm{E}+03$ grams

Source Length $=7.500 E+02 \mathrm{~cm} \quad$ Distance Along $C y 1$ inder, $Y=7.490 E+02 \mathrm{~cm}$

Integration Specs: $\quad$ NTHETA $=11$ NPSI $=11$ DELR computed internally

Total Intervals: $1.038 \mathrm{E}+05$ (photon source is the lst region)

Shield Thickness: $1.000 \mathrm{E}+00,6.350 \mathrm{E}-01,3.650 \mathrm{E}-01 \mathrm{~cm}$

Distances from Dose Point to the Outside of
(1) Source Region: $1.000 \mathrm{E}+00 \mathrm{~cm}$
(2) Next Layer: $3.650 \mathrm{E}-01 \mathrm{~cm}$

Dose Buildup Data for Shield 2 with Effective Atomic Number 26.00

Buildup Material is Iron

Source values are interpreted as $\mu \mathrm{C} \mathbf{i}$

Source Scale Factor was $1.000 E+03$

Fluence-to-Dose Conversion: Photons in Air

\begin{tabular}{|c|c|c|c|c|}
\hline $\begin{array}{c}\text { Average } \\
\mathrm{E}, \mathrm{MeV}\end{array}$ & $\begin{array}{r}\text { Source Total } \\
\text { photons/sec }\end{array}$ & $\begin{array}{l}\text { Fluence to } \\
\text { Dose Factor }\end{array}$ & $\begin{array}{c}\text { Energy Fluence } \\
\mathrm{MeV} / \mathrm{cm}^{2} / \mathrm{s}\end{array}$ & $\begin{array}{c}\text { Dose Rate } \\
\text { R/hr }\end{array}$ \\
\hline $\begin{array}{l}0.0131 \\
0.0245 \\
0.0354 \\
0.0429 \\
0.0548 \\
0.0659 \\
0.0748 \\
0.0819 \\
0.0933 \\
0.1522 \\
0.2310 \\
0.3458 \\
0.5068 \\
0.6423 \\
0.7680 \\
1.0434 \\
1.1417 \\
1.4187 \\
1.6904 \\
1.8897 \\
2.1850 \\
2.2879 \\
2.4864 \\
2.6844 \\
2.9209 \\
3.2000\end{array}$ & $\begin{array}{l}5.519 \mathrm{E}+07 \\
1.103 \mathrm{E}+06 \\
2.358 \mathrm{E}+06 \\
9.431 \mathrm{E}+05 \\
3.898 \mathrm{E}+05 \\
8.923 \mathrm{E}+05 \\
2.460 \mathrm{E}+05 \\
5.570 \mathrm{E}+05 \\
4.569 \mathrm{E}+05 \\
6.358 \mathrm{E}+06 \\
7.047 \mathrm{E}+05 \\
2.452 \mathrm{E}+05 \\
5.574 \mathrm{E}+05 \\
6.839 \mathrm{E}+06 \\
2.034 \mathrm{E}+07 \\
3.834 \mathrm{E}+05 \\
1.514 \mathrm{E}+05 \\
2.726 \mathrm{E}+05 \\
5.942 \mathrm{E}+03 \\
3.301 \mathrm{E}+03 \\
1.673 \mathrm{E}+05 \\
8.734 \mathrm{E}+02 \\
4.005 \mathrm{E}+02 \\
1.651 \mathrm{E}+02 \\
7.672 \mathrm{E}+01 \\
5.883 \mathrm{E}+00\end{array}$ & $\begin{array}{l}1.263 \mathrm{E}-04 \\
1.754 \mathrm{E}-05 \\
5.931 \mathrm{E}-06 \\
3.621 \mathrm{E}-06 \\
2.232 \mathrm{E}-06 \\
1.811 \mathrm{E}-06 \\
1.667 \mathrm{E}-06 \\
1.621 \mathrm{E}-06 \\
1.594 \mathrm{E}-06 \\
1.734 \mathrm{E}-06 \\
1.901 \mathrm{E}-06 \\
2.016 \mathrm{E}-06 \\
2.040 \mathrm{E}-06 \\
2.030 \mathrm{E}-06 \\
1.997 \mathrm{E}-06 \\
1.907 \mathrm{E}-06 \\
1.875 \mathrm{E}-06 \\
1.790 \mathrm{E}-06 \\
1.714 \mathrm{E}-06 \\
1.663 \mathrm{E}-06 \\
1.604 \mathrm{E}-06 \\
1.593 \mathrm{E}-06 \\
1.538 \mathrm{E}-06 \\
1.502 \mathrm{E}-06 \\
1.465 \mathrm{E}-06 \\
1.425 \mathrm{E}-06\end{array}$ & $\begin{array}{l}0.000 \mathrm{E}+00 \\
0.000 \mathrm{E}+00 \\
3.227 \mathrm{E}-12 \\
1.877 \mathrm{E}-07 \\
3.112 \mathrm{E}-04 \\
2.254 \mathrm{E}-02 \\
3.131 \mathrm{E}-02 \\
1.694 \mathrm{E}-01 \\
3.669 \mathrm{E}-01 \\
5.465 \mathrm{E}+01 \\
1.174 \mathrm{E}+01 \\
6.881 \mathrm{E}+00 \\
2.447 \mathrm{E}+02 \\
3.919 \mathrm{E}+02 \\
1.417 \mathrm{E}+03 \\
3.696 \mathrm{E}+01 \\
1.602 \mathrm{E}+01 \\
3.592 \mathrm{E}+01 \\
9.312 \mathrm{E}-01 \\
5.774 \mathrm{E}-01 \\
3.380 \mathrm{E}+01 \\
1.847 \mathrm{E}-01 \\
9.193 \mathrm{E}-02 \\
4.079 \mathrm{E}-02 \\
2.053 \mathrm{E}-02 \\
1.718 \mathrm{E}-03\end{array}$ & $\begin{array}{l}0.000 \mathrm{E}+00 \\
0.000 \mathrm{E}+00 \\
1.914 \mathrm{E}-17 \\
6.797 \mathrm{E}-13 \\
6.945 \mathrm{E}-10 \\
4.083 \mathrm{E}-08 \\
5.219 \mathrm{E}-08 \\
2.746 \mathrm{E}-07 \\
5.847 \mathrm{E}-07 \\
9.474 \mathrm{E}-05 \\
2.232 \mathrm{E}-05 \\
1.387 \mathrm{E}-05 \\
4.991 \mathrm{E}-04 \\
7.953 \mathrm{E}-04 \\
2.829 \mathrm{E}-03 \\
7.051 \mathrm{E}-05 \\
3.003 \mathrm{E}-05 \\
6.431 \mathrm{E}-05 \\
1.596 \mathrm{E}-06 \\
9.600 \mathrm{E}-07 \\
5.421 \mathrm{E}-05 \\
2.943 \mathrm{E}-07 \\
1.414 \mathrm{E}-07 \\
6.124 \mathrm{E}-08 \\
3.007 \mathrm{E}-08 \\
2.448 \mathrm{E}-09\end{array}$ \\
\hline Totals: & $1.032 \mathrm{E}+08$ & ons/sec & $2.252 \mathrm{E}+03$ & $4.478 \mathrm{E}-03$ \\
\hline
\end{tabular}


HNF-SD-WM-CN-091

Rev. 0

Page 57 of 64

New UNH Tank (H0-64-5473) Annular Source - End

Shield Composition, $\mathrm{g} / \mathrm{cc}$

Shield 1 Shield 2 Shield 3 Shield 4

\begin{tabular}{lllll}
\hline AIR & $1.290 \mathrm{E}-03$ & $0.000 \mathrm{E}+00$ & $0.000 \mathrm{E}+00$ & $1.290 \mathrm{E}-03$ \\
ALUMINUM & $0.000 \mathrm{E}+00$ & $1.665 \mathrm{E}+00$ & $0.000 \mathrm{E}+00$ & $0.000 \mathrm{E}+00$ \\
IRON & $0.000 \mathrm{E}+00$ & $0.000 \mathrm{E}+00$ & $7.800 \mathrm{E}+00$ & $0.000 \mathrm{E}+00$ \\
\hline Totals: & $1.290 \mathrm{E}-03$ & $1.665 \mathrm{E}+00$ & $7.800 \mathrm{E}+00$ & $1.290 \mathrm{E}-03$ \\
\hline
\end{tabular}

$E$, MeV Linear Attenuation, per $\mathrm{cm}$ (last region is air)

\begin{tabular}{lllll}
\hline 0.0131 & $2.838 \mathrm{E}-03$ & $2.004 \mathrm{E}+01$ & $6.779 \mathrm{E}+02$ & $2.838 \mathrm{E}-03$ \\
0.0245 & $6.409 \mathrm{E}-04$ & $3.229 \mathrm{E}+00$ & $1.146 \mathrm{E}+02$ & $6.409 \mathrm{E}-04$ \\
0.0354 & $3.627 \mathrm{E}-04$ & $1.236 \mathrm{E}+00$ & $4.037 \mathrm{E}+01$ & $3.627 \mathrm{E}-04$ \\
0.0429 & $2.979 \mathrm{E}-04$ & $8.096 \mathrm{E}-01$ & $2.318 \mathrm{E}+01$ & $2.979 \mathrm{E}-04$ \\
0.0548 & $2.497 \mathrm{E}-04$ & $5.145 \mathrm{E}-01$ & $1.182 \mathrm{E}+01$ & $2.497 \mathrm{E}-04$ \\
0.0659 & $2.302 \mathrm{E}-04$ & $4.008 \mathrm{E}-01$ & $7.416 \mathrm{E}+00$ & $2.302 \mathrm{E}-04$ \\
0.0748 & $2.186 \mathrm{E}-04$ & $3.516 \mathrm{E}-01$ & $5.442 \mathrm{E}+00$ & $2.186 \mathrm{E}-04$ \\
0.0819 & $2.117 \mathrm{E}-04$ & $3.254 \mathrm{E}-01$ & $4.420 \mathrm{E}+00$ & $2.117 \mathrm{E}-04$ \\
0.0933 & $2.030 \mathrm{E}-04$ & $2.947 \mathrm{E}-01$ & $3.335 \mathrm{E}+00$ & $2.030 \mathrm{E}-04$ \\
0.1522 & $1.749 \mathrm{E}-04$ & $2.284 \mathrm{E}-01$ & $1.508 \mathrm{E}+00$ & $1.749 \mathrm{E}-04$ \\
0.2310 & $1.518 \mathrm{E}-04$ & $1.920 \mathrm{E}-01$ & $1.031 \mathrm{E}+00$ & $1.518 \mathrm{E}-04$ \\
0.3458 & $1.304 \mathrm{E}-04$ & $1.636 \mathrm{E}-01$ & $7.918 \mathrm{E}-01$ & $1.304 \mathrm{E}-04$ \\
0.5068 & $1.114 \mathrm{E}-04$ & $1.399 \mathrm{E}-01$ & $6.509 \mathrm{E}-01$ & $1.114 \mathrm{E}-04$ \\
0.6423 & $1.006 \mathrm{E}-04$ & $1.259 \mathrm{E}-01$ & $5.802 \mathrm{E}-01$ & $1.006 \mathrm{E}-04$ \\
0.7680 & $9.281 \mathrm{E}-05$ & $1.160 \mathrm{E}-01$ & $5.316 \mathrm{E}-01$ & $9.281 \mathrm{E}-05$ \\
1.0434 & $8.021 \mathrm{E}-05$ & $1.001 \mathrm{E}-01$ & $4.565 \mathrm{E}-01$ & $8.021 \mathrm{E}-05$ \\
1.1417 & $7.665 \mathrm{E}-05$ & $9.565 \mathrm{E}-02$ & $4.360 \mathrm{E}-01$ & $7.665 \mathrm{E}-05$ \\
1.4187 & $6.855 \mathrm{E}-05$ & $8.565 \mathrm{E}-02$ & $3.906 \mathrm{E}-01$ & $6.855 \mathrm{E}-05$ \\
1.6904 & $6.267 \mathrm{E}-05$ & $7.831 \mathrm{E}-02$ & $3.585 \mathrm{E}-01$ & $6.267 \mathrm{E}-05$ \\
1.8897 & $5.906 \mathrm{E}-05$ & $7.401 \mathrm{E}-02$ & $3.402 \mathrm{E}-01$ & $5.906 \mathrm{E}-05$ \\
2.1850 & $5.431 \mathrm{E}-05$ & $6.883 \mathrm{E}-02$ & $3.188 \mathrm{E}-01$ & $5.431 \mathrm{E}-05$ \\
2.2879 & $5.280 \mathrm{E}-05$ & $6.728 \mathrm{E}-02$ & $3.124 \mathrm{E}-01$ & $5.280 \mathrm{E}-05$ \\
2.4864 & $5.019 \mathrm{E}-05$ & $6.458 \mathrm{E}-02$ & $3.017 \mathrm{E}-01$ & $5.019 \mathrm{E}-05$ \\
2.6844 & $4.795 \mathrm{E}-05$ & $6.221 \mathrm{E}-02$ & $2.926 \mathrm{E}-01$ & $4.795 \mathrm{E}-05$ \\
2.9209 & $4.577 \mathrm{E}-05$ & $5.971 \mathrm{E}-02$ & $2.836 \mathrm{E}-01$ & $4.577 \mathrm{E}-05$ \\
3.2000 & $4.383 \mathrm{E}-05$ & $5.717 \mathrm{E}-02$ & $2.751 \mathrm{E}-01$ & $4.383 \mathrm{E}-05$ \\
\hline & & & &
\end{tabular}


HNF-SD-WM-CN-091

Rev. 0

Page 58 of 64

New UNH Tank (H0-64-5473) Annular Source - End

Source Shields Distance to Detector, $X=7.350 E+01 \mathrm{~cm}$

Annular $13 \quad$ Cyl. \& S1ab Source Volume $=3.414 \mathrm{E}+04 \mathrm{cc}$

Source Mass $=5.684 \mathrm{E}+04$ grams

Source Length $=7.500 \mathrm{E}+02 \mathrm{~cm} \quad$ Distance Along Cylinder, $Y=7.490 \mathrm{E}+02 \mathrm{~cm}$

Integration Specs: $\quad$ NTHETA $=11$ NPSI $=11$ DELR computed internally

Total Intervals: $1.861 \mathrm{E}+02$ (photon source is the 2 nd region)

Shield Thickness: $7.240 \mathrm{E}+01,1.000 \mathrm{E}-01,6.350 \mathrm{E}-01,3.650 \mathrm{E}-01 \mathrm{~cm}$

Distances from Dose Point to the Outside of

(1) Source Region: $1.000 \mathrm{E}+00 \mathrm{~cm}$ (2) Next Layer: $3.650 \mathrm{E}-01 \mathrm{~cm}$

Dose Buildup Data for Shield 3 with Effective Atomic Number 26.00

Buildup Material is Iron

Source values are interpreted as $\mu \mathrm{C} i$

Source Scale Factor was $1.000 \mathrm{E}+03$

Fluence-to-Dose Conversion: Photons in Air

\begin{tabular}{ccccc}
$\begin{array}{r}\text { Average } \\
\text { E, MeV }\end{array}$ & $\begin{array}{c}\text { Source Total } \\
\text { photons } / \mathrm{sec}\end{array}$ & $\begin{array}{c}\text { Fluence to } \\
\text { Dose Factor }\end{array}$ & $\begin{array}{c}\text { Energy Fluence } \\
\text { MeV/cm } / \mathrm{s}\end{array}$ & $\begin{array}{c}\text { Dose Rate } \\
\text { R/hr }\end{array}$ \\
\hline 0.0131 & $5.519 \mathrm{E}+07$ & $1.263 \mathrm{E}-04$ & $0.000 \mathrm{E}+00$ & $0.000 \mathrm{E}+00$ \\
0.0245 & $1.103 \mathrm{E}+06$ & $1.754 \mathrm{E}-05$ & $0.000 \mathrm{E}+00$ & $0.000 \mathrm{E}+00$ \\
0.0354 & $2.358 \mathrm{E}+06$ & $5.931 \mathrm{E}-06$ & $7.029 \mathrm{E}-14$ & $4.169 \mathrm{E}-19$ \\
0.0429 & $9.431 \mathrm{E}+05$ & $3.621 \mathrm{E}-06$ & $3.306 \mathrm{E}-09$ & $1.197 \mathrm{E}-14$ \\
0.0548 & $3.898 \mathrm{E}+05$ & $2.232 \mathrm{E}-06$ & $4.878 \mathrm{E}-06$ & $1.089 \mathrm{E}-11$ \\
0.0659 & $8.923 \mathrm{E}+05$ & $1.811 \mathrm{E}-06$ & $3.569 \mathrm{E}-04$ & $6.466 \mathrm{E}-10$ \\
0.0748 & $2.460 \mathrm{E}+05$ & $1.667 \mathrm{E}-06$ & $5.154 \mathrm{E}-04$ & $8.591 \mathrm{E}-10$ \\
0.0819 & $5.570 \mathrm{E}+05$ & $1.621 \mathrm{E}-06$ & $2.894 \mathrm{E}-03$ & $4.691 \mathrm{E}-09$ \\
0.0933 & $4.569 \mathrm{E}+05$ & $1.594 \mathrm{E}-06$ & $6.663 \mathrm{E}-03$ & $1.062 \mathrm{E}-08$ \\
0.1522 & $6.358 \mathrm{E}+06$ & $1.734 \mathrm{E}-06$ & $1.272 \mathrm{E}+00$ & $2.205 \mathrm{E}-06$ \\
0.2310 & $7.047 \mathrm{E}+05$ & $1.901 \mathrm{E}-06$ & $3.038 \mathrm{E}-01$ & $5.775 \mathrm{E}-07$ \\
0.3458 & $2.452 \mathrm{E}+05$ & $2.016 \mathrm{E}-06$ & $1.903 \mathrm{E}-01$ & $3.835 \mathrm{E}-07$ \\
0.5068 & $5.574 \mathrm{E}+06$ & $2.040 \mathrm{E}-06$ & $7.062 \mathrm{E}+00$ & $1.441 \mathrm{E}-05$ \\
0.6423 & $6.839 \mathrm{E}+06$ & $2.030 \mathrm{E}-06$ & $1.157 \mathrm{E}+01$ & $2.349 \mathrm{E}-05$ \\
0.7680 & $2.034 \mathrm{E}+07$ & $1.997 \mathrm{E}-06$ & $4.254 \mathrm{E}+01$ & $8.494 \mathrm{E}-05$ \\
1.0434 & $3.834 \mathrm{E}+05$ & $1.907 \mathrm{E}-06$ & $1.138 \mathrm{E}+00$ & $2.170 \mathrm{E}-06$ \\
1.1417 & $1.514 \mathrm{E}+05$ & $1.875 \mathrm{E}-06$ & $4.963 \mathrm{E}-01$ & $9.306 \mathrm{E}-07$ \\
1.4187 & $2.726 \mathrm{E}+05$ & $1.790 \mathrm{E}-06$ & $1.129 \mathrm{E}+00$ & $2.021 \mathrm{E}-06$ \\
1.6904 & $5.942 \mathrm{E}+03$ & $1.714 \mathrm{E}-06$ & $2.953 \mathrm{E}-02$ & $5.063 \mathrm{E}-08$ \\
1.8897 & $3.301 \mathrm{E}+03$ & $1.663 \mathrm{E}-06$ & $1.840 \mathrm{E}-02$ & $3.059 \mathrm{E}-08$ \\
2.1850 & $1.673 \mathrm{E}+05$ & $1.604 \mathrm{E}-06$ & $1.083 \mathrm{E}+00$ & $1.738 \mathrm{E}-06$ \\
2.2879 & $8.734 \mathrm{E}+02$ & $1.593 \mathrm{E}-06$ & $5.931 \mathrm{E}-03$ & $9.449 \mathrm{E}-09$ \\
2.4864 & $4.005 \mathrm{E}+02$ & $1.538 \mathrm{E}-06$ & $2.959 \mathrm{E}-03$ & $4.550 \mathrm{E}-09$ \\
2.6844 & $1.651 \mathrm{E}+02$ & $1.502 \mathrm{E}-06$ & $1.314 \mathrm{E}-03$ & $1.974 \mathrm{E}-09$ \\
2.9209 & $7.672 \mathrm{E}+01$ & $1.465 \mathrm{E}-06$ & $6.621 \mathrm{E}-04$ & $9.697 \mathrm{E}-10$ \\
3.2000 & $5.883 \mathrm{E}+00$ & $1.425 \mathrm{E}-06$ & $5.543 \mathrm{E}-05$ & $7.898 \mathrm{E}-11$ \\
\hline
\end{tabular}

Totals: $\quad 1.032 \mathrm{E}+08$ photons $/ \mathrm{sec} \quad 6.685 \mathrm{E}+01 \quad 1.330 \mathrm{E}-04 \mathrm{R} / \mathrm{hr}$

Closing: DAT'S ALL PHOLQUES!!!!!!! 
Finish run at $11: 55: 31 \quad 12 / 20 / 96$

Input File ( $D: \backslash$ ISOSHLD INPUT\TANKER.) is shown below:

0

Organic Tank (H0-64-4278) Line Source - Midd7e

\& INPUT NEXT $=1$, IGEOM $=7$, NTHETA $=11$, NPS $I=11$, $N S H L D=2, J B U F=2, I C O N C=0, O P T I O N=1$,

$\mathrm{SLTH}=1036 ., \mathrm{Y}=518, \mathrm{X}=2.5, \mathrm{~T}(1)=1 ., \mathrm{T}(2)=1.27$, SRCUNIT $=0$, DOSEUNIT=0, SRCPRFX $=-6$, SFACT $=1.0 E+3$, WEIGHT $(376)=7.12 \mathrm{E}-01$, WEIGHT $(377)=7.12 \mathrm{E}-01$, WEIGHT $(472)=1.45 E-01$, WEIGHT $(319)=1.93 E-02$, WEIGHT $(335)=8.45 \mathrm{E}-01$, WEIGHT $(336)=7.99 \mathrm{E}-01$, WEIGHT $(119)=1.84 \mathrm{E}-02$, WEIGHT $(155)=1.46 \mathrm{E}-02$, WEIGHT $(170)=6.82 \mathrm{E}+00$, WEIGHT $(172)=6.82 \mathrm{E}+00$, WEIGHT $(117)=2.08 \mathrm{E}-02$, WEIGHT $(496)=2.15 \mathrm{E}-02$, WEIGHT $(418)=8.44 E-02$, WEIGHT $(269)=1.20 E-01$, WEIGHT $(520)=1.72 \mathrm{E}-09$, WEIGHT $(398)=4.97 \mathrm{E}-08$, $\operatorname{WEIGHT}(476)=1.39 \mathrm{E}-08$, WEIGHT $(526)=8.31 \mathrm{E}-06, \quad \&$ SOURCE 70.8122

1 STEEL $9 \quad 7.8$

Organic Tank (H0-64-4278) Annular Source - Middle \& INPUT NEXT $=1$, IGEOM=13, NTHETA $=11$, NPS $I=11$, NSHLD $=3, J B U F=3, I C O N C=0$, OPTION $=0, T(3)=1.27$, $S L T H=1036 ., Y=518 ., X=78 ., T(1)=76.2, T(2)=0.1$, SRCUNIT $=0$, DOSEUNIT $=0$, SRCPRFX $=-6$, SFACT $=1.0 \mathrm{E}+3$, $\operatorname{WEIGHT}(376)=7.12 \mathrm{E}-01$, WEIGHT $(377)=7.12 \mathrm{E}-01$, $\operatorname{WEIGHT}(472)=1.45 \mathrm{E}-01$, WEIGHT $(319)=1.93 \mathrm{E}-02$, WEIGHT $(335)=8.45 \mathrm{E}-01$, WEIGHT $(336)=7.99 \mathrm{E}-01$, WEIGHT $(119)=1.84 E-02$, WEIGHT $(155)=1.46 E-02$, WEIGHT $(170)=6.82 E+00$, WEIGHT $(172)=6.82 E+00$, WEIGHT $(117)=2.08 E-02$, WEIGHT $(496)=2.15 E-02$, WEIGHT (418) $=8.44 E-02$, WEIGHT $(269)=1.20 \mathrm{E}-01$, WEIGHT $(520)=1.72 E-09$, WEIGHT $(398)=4.97 E-08$, WEIGHT $(476)=1.39 E-08$, WEIGHT $(526)=8.31 E-06, \&$ AIR 3.00129 $\begin{array}{ll}\text { SOURCE } 7 & 0.8122\end{array}$

1 STEEL 9 7.8 
Organic Tank (HO-64-4278) Line Source - End

\&INPUT NEXT $=1, I G E O M=7$, NTHETA $=11$, NPSI $=11$, $N S H L D=2, J B U F=2, I C O N C=0$, OPTION $=0$, $S L T H=1036 ., Y=1035 ., X=2.5, T(1)=1 ., T(2)=1.27$,

SRCUNIT $=0$, DOSEUNIT $=0$, SRCPRFX $=-6$, SFACT $=1.0 E+3$, WEIGHT $(376)=7.12 \mathrm{E}-01$, WEIGHT $(377)=7.12 \mathrm{E}-01$, WEIGHT $(472)=1.45 \mathrm{E}-01$, WEIGHT $(319)=1.93 \mathrm{E}-02$, $\operatorname{WEIGHT}(335)=8.45 \mathrm{E}-01$, WEIGHT $(336)=7.99 \mathrm{E}-01$, WEIGHT $(119)=1.84 \mathrm{E}-02$, WEIGHT $(155)=1.46 \mathrm{E}-02$, WEIGHT $(170)=6.82 E+00$, WEIGHT $(172)=6.82 E+00$, WEIGHT $(117)=2.08 \mathrm{E}-02$, WEIGHT $(496)=2.15 \mathrm{E}-02$, WEIGHT $(418)=8.44 \mathrm{E}-02$, WEIGHT $(269)=1.20 \mathrm{E}-01$, WEIGHT $(520)=1.72 \mathrm{E}-09$, WEIGHT $(398)=4.97 \mathrm{E}-08$, WEIGHT $(476)=1.39 \mathrm{E}-08$, WEIGHT $(526)=8.31 \mathrm{E}-06$, \& SOURCE 70.8122

1 STEEL 9 7.8

Organic Tank (H0-64-4278) Annular Source - End \&INPUT NEXT $=1, \quad$ IGEOM $=13$, NTHETA $=11, N P S I=11$, $\mathrm{NSHLD}=3, \mathrm{JBUF}=3, \mathrm{ICONC}=0, \mathrm{OPTION}=0, \mathrm{~T}(3)=1.27$, $S L T H=1036 ., Y=1035 ., X=78 ., T(1)=76.2, T(2)=0.1$,

SRCUNIT $=0$, DOSEUNIT $=0, \quad$ SRCPRFX $=-6, \quad S F A C T=1.0 \mathrm{E}+3$, $\operatorname{WEIGHT}(376)=7.12 \mathrm{E}-01$, WEIGHT (377) $=7.12 \mathrm{E}-01$, WEIGHT $(472)=1.45 E-01$, WEIGHT $(319)=1.93 E-02$, WEIGHT $(335)=8.45 E-01$, WEIGHT $(336)=7.99 E-01$, WEIGHT $(119)=1.84 \mathrm{E}-02$, WEIGHT $(155)=1.46 \mathrm{E}-02$, WEIGHT $(170)=6.82 E+00$, WEIGHT $(172)=6.82 \mathrm{E}+00$, WEIGHT $(117)=2.08 \mathrm{E}-02$, WEIGHT $(496)=2.15 \mathrm{E}-02$, WE IGHT (418) $=8.44 E-02$, WE IGHT $(269)=1.20 E-01$, WEIGHT $(520)=1.72 \mathrm{E}-09$, WEIGHT $(398)=4.97 \mathrm{E}-08$, WEIGHT $(476)=1.39 E-08$, WEIGHT $(526)=8.31 E-05$, \& AIR $\quad 3.00129$

SOURCE 7 O 0.8122

1 STEEL 9 
01d UNH Tank (HO-64-5920) Line Source - Middle \&INPUT NEXT $=1$, IGEOM=7, NTHETA $=11$, NPSI $=11$, NSHLD $=2, J B U F=2, I C O N C=0, O P T I O N=1$, $S L T H=701, Y=350.5, X=2 ., T(1)=1 ., T(2)=0.635$, SRCUNIT $=0$, DOSEUNIT $=0$, SRCPRFX $=-6$, SFACT $=1.0 E+3$, WEIGHT $(376)=5.81 \mathrm{E}-01$, WEIGHT $(377)=5.81 \mathrm{E}-0 \mathrm{l}$, WEIGHT (319) $=4.27 \mathrm{E}-02$, WEIGHT $(335)=3.60 \mathrm{E}-02$, WEIGHT $(336)=3.41 \mathrm{E}-02$, WEIGHT $(119)=4.67 \mathrm{E}-01$, WEIGHT $(155)=3.96 \mathrm{E}-02$, WEIGHT $(170)=5.33 \mathrm{E}-01$, WEIGHT $(172)=5.33 \mathrm{E}-01$, WEIGHT $(117)=7.18 \mathrm{E}-02$, WEIGHT $(526)=1.59 \mathrm{E}+01$, WEIGHT $(398)=2.17 \mathrm{E}-02$, WEIGHT $(476)=9.80 \mathrm{E}-02$, WEIGHT $(520)=2.69 \mathrm{E}-03$, WEIGHT $(472)=0.00 E+00$, WEIGHT $(496)=0.00 E+00$, WEIGHT (418) $=0.00 \mathrm{E}+00$, WEIGHT (269) $=0.00 \mathrm{E}+00$, \& SOURCE 71.665

1 STEEL 9 7.8

01d UNH Tank (H0-64-5920) Annular Source - Middle \& INPUT NEXT $=1, I G E O M=13$, NTHETA $=11$, NPS $1=11$, NSHLD $=3$, JBUF $=3$, ICONC $=0,0 P T I O N=0, T(3)=0.635$, $\mathrm{SLTH}=701 ., Y=350.5, X=73.5, \mathrm{~T}(1)=72.4, \mathrm{~T}(2)=0.1$, SRCUNIT $=0$, DOSEUNIT $=0$, SRCPRFX $=-6$, SFACT $=1.0 \mathrm{E}+3$, WEIGHT $(376)=5.81 \mathrm{E}-01$, WEIGHT $(377)=5.81 \mathrm{E}-01$, WEIGHT $(319)=4.27 \mathrm{E}-02$, WEIGHT $(335)=3.60 \mathrm{E}-02$, WEIGHT $(336)=3.41 E-02$, WEIGHT $(119)=4.67 E-01$, WEIGHT $(155)=3.96 \mathrm{E}-02$, WEIGHT $(170)=5.33 \mathrm{E}-01$, WEIGHT $(172)=5.33 \mathrm{E}-01$, WEIGHT $(117)=7.18 \mathrm{E}-02$, WEIGHT $(526)=1.59 E+01$, WEIGHT $(398)=2.17 E-02$, WEIGHT $(476)=9.80 E-02$, WEIGHT $(520)=2.69 \mathrm{E}-03$, WEIGHT $(472)=0.00 E+00$, WEIGHT $(496)=0.00 E+00$, WEIGHT (418) $=0.00 E+00$, WEIGHT $(269)=0.00 E+00$, \& AIR $\quad 3.00129$ SOURCE $7 \quad 1.665$

1 STEEL 9 7.8

01d UNH Tank (H0-64-5920) Line Source - End \&INPUT NEXT $=1, \mathrm{I}$ GEOM=7, NTHETA $=11$, NPSI $=11$, NSHLD $=2, J B U F=2, I C O N C=0, O P T I O N=0$, $\mathrm{SLTH}=701 ., Y=700 ., X=2 ., T(1)=1 ., T(2)=0.635$, SRCUNIT $=0$, DOSEUNIT $=0$, SRCPRFX $=-6$, SFACT $=1.0 \mathrm{E}+3$, WEIGHT $(376)=5.81 \mathrm{E}-01$, WEIGHT $(377)=5.81 \mathrm{E}-01$, WEIGHT $(319)=4.27 \mathrm{E}-02$, WEIGHT $(335)=3.60 \mathrm{E}-02$, WEIGHT (336) $=3.41 \mathrm{E}-02$, WEIGHT $(119)=4.67 \mathrm{E}-01$, WEIGHT $(155)=3.96 \mathrm{E}-02$, WEIGHT $(170)=5.33 \mathrm{E}-01$, WEIGHT $(172)=5.33 \mathrm{E}-01$, WEIGHT $(117)=7.18 \mathrm{E}-02$, WEIGHT $(526)=1.59 \mathrm{E}+01$, WEIGHT $(398)=2.17 \mathrm{E}-02$, WEIGHT $(476)=9.80 \mathrm{E}-02$, WEIGHT $(520)=2.69 \mathrm{E}-03$, WEIGHT (472) $=0.00 E+00$, WEIGHT $(496)=0.00 E+00$, WEIGHT $(418)=0.00 E+00$, WEIGHT $(269)=0.00 E+00$, \& SOURCE 71.665

1 STEEL $9 \quad 7.8$ 
01d UNH Tank (H0-64-5920) Annular Source - End \&INPUT NEXT $=1$, IGEOM $=13$, NTHETA $=11$, NPSI $=11$, NSHLD $=3, J B U F=3$, ICONC $=0$, OPTION $=0, T(3)=0.635$, $\mathrm{SLTH}=701 ., Y=700 ., X=73.5, \mathrm{~T}(1)=72.4, \mathrm{~T}(2)=0.1$, SRCUNIT $=0$, DOSEUNIT $=0$, SRCPRFX $=-6$, SFACT $=1.0 \mathrm{E}+3$, $\operatorname{WEIGHT}(376)=5.81 \mathrm{E}-01$, WEIGHT $(377)=5.81 \mathrm{E}-01$, WEIGHT (319) $=4.27 \mathrm{E}-02$, WEIGHT $(335)=3.60 \mathrm{E}-02$, WEIGHT $(336)=3.41 \mathrm{E}-02$, WEIGHT $(119)=4.67 \mathrm{E}-01$, WEIGHT $(155)=3.96 \mathrm{E}-02$, WEIGHT $(170)=5.33 \mathrm{E}-01$, WEIGHT $(172)=5.33 \mathrm{E}-01$, WEIGHT $(117)=7.18 \mathrm{E}-02$, WEIGHT $(526)=1.59 E+01$, WEIGHT $(398)=2.17 \mathrm{E}-02$, WEIGHT $(476)=9.80 E-02$, WEIGHT $(520)=2.69 E-03$, $W E I G H T(472)=0.00 E+00$, WEIGHT $(495)=0.00 E+00$, WEIGHT $(418)=0.00 E+00$, WEIGHT $(269)=0.00 E+00, \&$ AIR $\quad 3.00129$ SOURCE $7 \quad 1.665$

1 STEEL 9

New UNH Tank (H0-64-5473) Line Source - Middle \& INPUT NEXT $=1$, I GEOM $=7$, NTHETA $=11$, NPS I $=11$, NSHLD $=2, J B U F=2, I C O N C=0$, OPTION $=1$, $S L T H=750 ., Y=375 ., X=2 ., T(1)=1 ., T(2)=0.635$, SRCUNIT $=0$, DOSEUN IT $=0$, SRCPRFX $=-6$, SFACT $=1.0 \mathrm{E}+3$, WEIGHT $(376)=5.81 E-01$, WEIGHT $(377)=5.81 \mathrm{E}-01$, WEIGHT $(319)=4.27 \mathrm{E}-02$, WEIGHT $(335)=3.60 \mathrm{E}-02$, WEIGHT $(336)=3.41 E-02$, WEIGHT $(119)=4.67 E-01$, WEIGHT $(155)=3.96 \mathrm{E}-02$, WEIGHT $(170)=5.33 \mathrm{E}-01$, WEIGHT $(172)=5.33 \mathrm{E}-01$, WEIGHT $(117)=7.18 \mathrm{E}-02$, WEIGHT $(526)=1.59 E+01$, WEIGHT $(398)=2.17 E-02$, WEIGHT $(476)=9.80 E-02, W E I G H T(520)=2.69 E-03$, WEIGHT $(472)=0.00 E+00$, WEIGHT $(496)=0.00 E+00$, $\operatorname{WEIGHT}(418)=0.00 \mathrm{E}+00, \operatorname{WEIGHT}(269)=0.00 \mathrm{E}+00, \&$ SOURCE $7 \quad 1.665$

\section{STEEL $9 \quad 7.8$}

New UNH Tank (H0-64-5473) Annular Source - Middle \& INPUT NEXT $=1,1 \mathrm{GEOM}=13, \mathrm{NTHETA}=11$, NPS I $=11$, $N S H L D=3, J B U F=3, I C O N C=0, O P T I O N=0, T(3)=0.635$, $S L T H=750 ., \quad Y=375 ., X=73.5, T(1)=72.4, T(2)=0.1$, SRCUNIT $=0$, DOSEUNIT $=0$, SRCPRFX $=-6$, SFACT $=1.0 E+3$, WEIGHT $(376)=5.81 \mathrm{E}-01$, WEIGHT $(377)=5.81 \mathrm{E}-01$, WEIGHT $(319)=4.27 \mathrm{E}-02$, WEIGHT $(335)=3.60 \mathrm{E}-02$, WEIGHT $(336)=3.41 E-02$, WEIGHT $(119)=4.67 E-01$, WEIGHT $(155)=3.96 E-02$, WEIGHT $(170)=5.33 E-01$, WEIGHT $(172)=5.33 E-01$, WEIGHT $(117)=7.18 \mathrm{E}-02$, WEIGHT $(526)=1.59 E+01$, WEIGHT $(398)=2.17 E-02$, WEIGHT (476) $=9.80 E-02$, WEIGHT $(520)=2.69 E-03$, WEIGHT $(472)=0.00 E+00$, WEIGHT $(496)=0.00 E+00$, WEIGHT (418) $=0.00 E+00, W E I G H T(269)=0.00 E+00, \&$ AIR 3.00129 SOURCE $7 \quad 1.665$ 
New UNH Tank (H0-64-5473) Line Source - End \&INPUT NEXT $=1$, I GEOM $=7$, NTHETA $=11$, NPSI $=11$, NSHLD $=2, J B U F=2$, ICONC $=0$, OPTION $=0$, $S L T H=750 ., Y=749 ., X=2 ., T(1)=1 ., T(2)=0.635$, SRCUNIT $=0$, DOSEUNIT $=0$, SRCPRFX $=-6$, SFACT $=1.0 \mathrm{E}+3$, WEIGHT $(376)=5.81 \mathrm{E}-01, \mathrm{WEIGHT}(377)=5.81 \mathrm{E}-01$, WEIGHT $(319)=4.27 \mathrm{E}-02$, WEIGHT $(335)=3.60 \mathrm{E}-02$, WEIGHT $(336)=3.41 \mathrm{E}-02$, WEIGHT $(119)=4.67 \mathrm{E}-01$, WEIGHT $(155)=3.96 \mathrm{E}-02$, WEIGHT $(170)=5.33 \mathrm{E}-01$, WEIGHT $(172)=5.33 \mathrm{E}-01$, WEIGHT $(117)=7.18 \mathrm{E}-02$, WEIGHT $(526)=1.59 E+01$, WEIGHT $(398)=2.17 \mathrm{E}-02$, WEIGHT $(476)=9.80 E-02$, WEIGHT $(520)=2.69 E-03$, WEIGHT $(472)=0.00 E+00$, WEIGHT $(496)=0.00 E+00$, WEIGHT $(418)=0.00 E+00, W E I G H T(269)=0.00 E+00, \&$ SOURCE $7 \quad 1.665$

1 STEEL 9 7.8

New UNH Tank (H0-64-5473) Annular Source - End

\& INPUT NEXT $=1,1 \mathrm{GEOM}=13, \mathrm{NTHETA}=11$, NPSI $=11$, NSHLD $=3, J B U F=3, I C O N C=0$, OPTION $=0, T(3)=0.635$, $S L T H=750 ., Y=749 ., X=73.5, T(1)=72.4, T(2)=0.1$, SRCUNIT $=0$, DOSEUNIT $=0$, SRCPRFX $=-6, \quad S F A C T=1.0 E+3$, WEIGHT $(376)=5.81 \mathrm{E}-01$, WEIGHT $(377)=5.81 \mathrm{E}-01$, WEIGHT $(319)=4.27 \mathrm{E}-02$, WEIGHT $(335)=3.60 \mathrm{E}-02$, WEIGHT $(336)=3.41 \mathrm{E}-02$, WEIGHT (119) $=4.67 \mathrm{E}-01$, $W E I G H T(155)=3.96 E-02$, WEIGHT $(170)=5.33 E-01$, WEIGHT $(172)=5.33 \mathrm{E}-01$, WEIGHT $(117)=7.18 \mathrm{E}-02$, WEIGHT $(526)=1.59 E+01$, WEIGHT (398) $=2.17 E-02$, WEIGHT $(476)=9.80 E-02$, WEIGHT $(520)=2.69 E-03$, WEIGHT $(472)=0.00 E+00$, WEIGHT $(496)=0.00 E+00$, WEIGHT $(418)=0.00 E+00, W E I G H T(269)=0.00 E+00, \&$ AIR 3.00129 SOURCE $7 \quad 1.665$

1 STEEL 9 7.8

DAT'S ALL PHOLQUES!!!!!!!!

\&INPUT NEXT $=6$, \& 
HNF-SD-WM-CN-091

Rev. 0

Page 64 of 64

\section{CHECKLIST FOR INDEPENDENT TECHNICAL REVIEW}

DOCUMENT REVIEWED

NUMBER: WHC-SD-WM-CN-087 Rev 0.

TITLE: Shielding Analys is for the WESF Ion Exchange Module

Reviewer(s): Steve Gedeon

I. Method(s) of Review

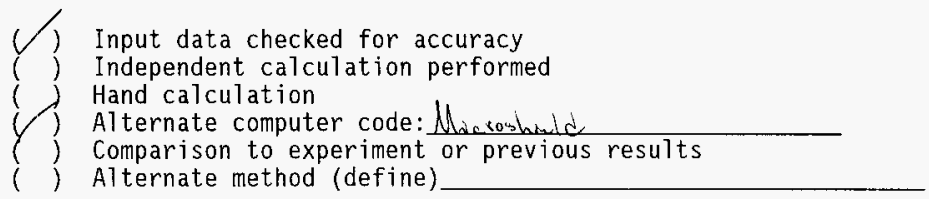

II. Checklist (either check or enter NA if not applied)

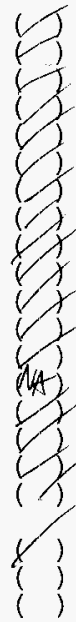

Task completely defined

Activity consistent with task specification

Necessary assumptions explicitly stated and supported

Resources properly identified and referenced

Resource documentation appropriate for this application

Input data explicitly stated

Input data verified to be consistent with original source

Geometric model adequate representation of actual geometry

Material properties appropriate and reasonable

Mathematical derivations checked including dimensional consistency

Hand calculations checked for errors

Assumptions explicitly stated and justified

Computer software appropriate for task and used within range of validity

Use of resource outside range of established validity is justified

Software runstreams correct and consistent with results

Software output consistent with input

Results consistent with applicable previous experimental or analytical findings

Results and conclusions address a 11 points and are consistent with task

requirements and/or established limits or criteria

Conclusions consistent with analytical results and established limits

Uncertainty assessment appropriate and reasonable

other (define)

III. Comments:

IV.

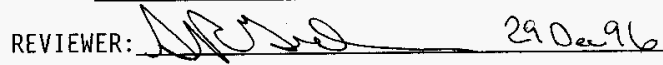




\begin{tabular}{|c|c|c|c|c|c|}
\hline \multicolumn{6}{|c|}{ DISTRIBUTION SHEET } \\
\hline & \multirow{2}{*}{\multicolumn{3}{|c|}{$\begin{array}{l}\text { From } \\
\text { Criticality and Shielding }\end{array}$}} & \multicolumn{2}{|l|}{ Page 1 of 1} \\
\hline Distribution & & & & \multicolumn{2}{|c|}{$\begin{array}{l}\text { Date } \\
\text { December } 30,1996\end{array}$} \\
\hline \multicolumn{4}{|l|}{ Project Title/Work Order } & \multicolumn{2}{|c|}{ EDT No. 620284} \\
\hline \multicolumn{4}{|c|}{$\begin{array}{l}\text { Calculational Note in Support of Inventory Assessment of Three } \\
\text { Tank Trailers Using Radiological Attenuation Calculations }\end{array}$} & \multicolumn{2}{|l|}{ ECN No. } \\
\hline Name & MSIN & $\begin{array}{c}\text { Text } \\
\text { With All } \\
\text { Attach. }\end{array}$ & Text Only & $\begin{array}{l}\text { Attach./ } \\
\text { Appendix } \\
\text { Only }\end{array}$ & $\begin{array}{l}\text { EDT/ECN } \\
\text { Only }\end{array}$ \\
\hline D. F. Baker & S6-01 & $x$ & & & \\
\hline S. R. Gedeon & HO-35 & $x$ & & & \\
\hline H. J. Goldberg & HO-35 & $x$ & & & \\
\hline J. Greenborg & $\mathrm{HO}-35$ & $x$ & & & \\
\hline J. R. Hilliard & S6-01 & $x$ & & & \\
\hline C. V. Villalobos & $56-19$ & $x$ & & & \\
\hline Central Files $(2+$ Original $)$ & $A 3-88$ & $x$ & & & \\
\hline Docket Files (2) & $\mathrm{B} 1-17$ & $x$ & & & \\
\hline
\end{tabular}

